\title{
A new approach toward the synthesis of 2,4-bis(fluoroalkyl)- substituted quinoline derivatives using Fluoroalkyl Amino Reagent chemistry
}

\author{
Fallia Aribi, ${ }^{a, d}$ Etienne Schmitt, ${ }^{\text {a,d }}$ Armen Panossian, ${ }^{\text {a,d }}$ Jean-Pierre Vors, ${ }^{b, d}$ Sergiy Pazenok, ${ }^{c, d}$ and \\ Frédéric R. Leroux ${ }^{*}$,d \\ The present work describes the unprecedented use of Fluoroalkyl Amino Reagents (FARs) to afford 2,4-bis(fluoroalkyl)- \\ substituted quinoline derivatives in two steps. In contrast to the Combes reaction, this approach allows for the synthesis of \\ numerous quinoline derivatives bearing two identical or different fluoroalkyl substituents in 2 and 4 positions, under mild \\ reaction conditions, in good yields and with a very good regioselectivity. This reaction is easily scalable and suitable for an \\ industrial process.
}

\section{Introduction}

Since the last decade, fluorine is starting to become an alternative solution to many issues in agrochemistry, medicinal chemistry and material science. Indeed, its specific properties such as its high electronegativity, the strength of the C-F bond due to its low polarizability, and its size comparable to that of a hydrogen atom gives it a special appeal. Introduction of fluorine into a lead molecule may considerably improve its physicochemical properties. For example, the metabolic stability of $\mathrm{C}-\mathrm{F}$ bonds toward oxidative degradation is wellknown, and the acidity or basicity of adjacent functional groups can be modified. Likewise, the lipophilicity of molecules can be enhanced by introducing fluorine atoms in order to facilitate transport through membranes. ${ }^{1-18}$ Therefore, the interest in fluorine chemistry encounters a swift rise and developing new strategies for the introduction of emergent fluorinated groups remains a challenge to overcome. A recent survey has shown that $20 \%$ of pharmaceuticals and $40 \%$ of agrochemicals (e.g. Fluazinam (fungicide), (Fluoxetine (antidepressant)) are F-containing compounds (Figure 1). Among the $155 \mathrm{~F}$-containing compounds listed as agrochemicals, 43\% bear a trifluoromethyl group, $32 \%$ a single fluorine atom and around $11 \%$ are difluoromethyl derivatives. ${ }^{19,} 20$ On the other hand, the quinoline motif is present in many natural products and drugs already commercialized (e.g. Mefloquine, used as antimalarial drug). For example, they are used in the treatment of cancer, inflammatory diseases, bacterial or viral infections, etc. Quinoline-derived molecules are known for their various biological activities which make them important pharmaceutical and agrochemical precursors. ${ }^{21-27}$ The combination of an aza-heterocycle and various fluorinated substituents may enhance the properties of previously known compounds and make them highly attractive as agrochemical ingredients. ${ }^{15,}$ 28-31

In this paper, we report on a new method to synthesize unprecedented 2,4-bis(fluoroalkyl)-substituted quinoline derivatives, based on the reaction of Fluoroalkyl Amino Reagents (FARs) (Scheme 1) with $N$-aryl imines 1. FARs represent a powerful tool for the introduction of various fluorinated substituents. In literature, three different FARs were used: 1,1,2,2-tetrafluoro- $N, N$-dimethylethan-1-amine (4a; TFEDMA), 2-chloro- $N, N$-diethyl-1,1,2-trifluoroethan-1amine (4b; Yarovenko reagent), and $N, N$-diethyl-1,1,2,3,3,3hexafluoropropan-1-amine (4c; Ishikawa reagent). FARs are prepared from commercially available fluoroolefins and dimethyl- or diethylamine, and were initially used as fluorinating agents in order to convert hydroxyl or carbonyl groups into fluoroakyl substituents under mild conditions. ${ }^{32-36}$

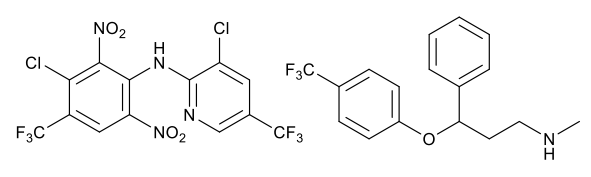

Fluazinam (Fungicide)

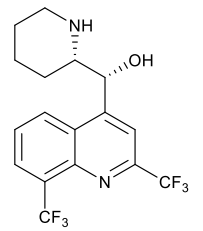

Mefloquine (Anti-malarial)
Figure 1: Bioactive compounds containing fluorinated groups.

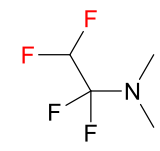

\section{$4 a$}

1,1,2,2-Tetrafluoro- $N, N$-dimethylethan-1-amine Petrov: TFEDMA (2001)

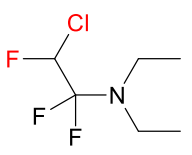

4b

2-Chloro- $N, N$-diethyl-1,1,2-trifluoroethan-1-amine Yarovenko reagent (1959)

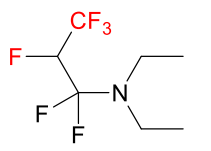

4c

$N, N$-Diethyl-1,1,2,3,3,3-hexafluoropropan-1-amine Ishikawa reagent (1979)

Scheme 1: Commercially available Fluoroalkyl Amino Reagents (FARs) 
A key feature of FARs is their activation by Lewis acids (e.g. $\mathrm{BF}_{3} \bullet \mathrm{Et}_{2} \mathrm{O}$ ), preferentially in an aprotic solvent, to provide highly reactive Vilsmeier-like iminium salts (Scheme 2).37-39 After activation, the electrophilic character of FARs is enhanced, and the iminium salts can undergo nucleophilic attack by various compounds.

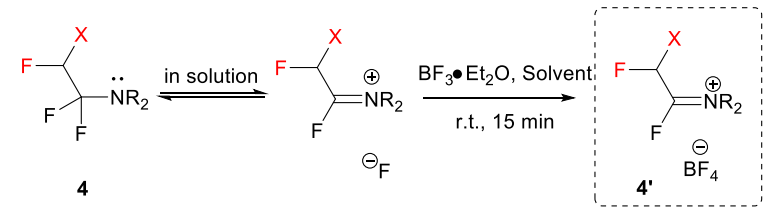

$\mathrm{R}=\mathrm{CH}_{3}, \mathrm{C}_{2} \mathrm{H}_{5}$

$\mathrm{X}=\mathrm{F}, \mathrm{Cl}, \mathrm{CF}_{3}$

Solvent: DCM, MeCN

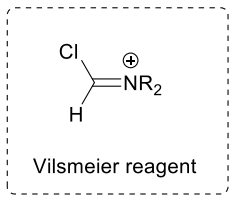

Scheme 2: Activation of FARs by Lewis acids converting them into their corresponding iminium salts.

Of the three existent FARs, TFEDMA is the most stable at ambient temperature, the purer after activation and it is also the most reactive one, due to the presence of the dimethyl moiety that makes it less hindered and thus more prone to nucleophilic attack.

In 1979, Ishikawa employed the Yarovenko reagent (4b) in cyclization reactions with 2-amino-anilines, -phenols and thiophenols to provide various benzazoles. ${ }^{40}$ More recently, we further demonstrated the ability of FARs to behave as $\alpha$ fluoroacyl equivalents for the introduction of fluorinated substituents $\left(\mathrm{CHF}_{2}, \mathrm{CHFCl}\right.$ and $\left.\mathrm{CHFCF}_{3}\right)$ in the preparation of a new family of compounds for agrochemistry. ${ }^{41-46}$ We were able to synthesize fluorinated heterocycles, especially 3,5bis(fluoroalkyl) pyrazoles following 3 strategies (Scheme 3). In Path a, pyrazoles carboxylates were formed by reaction of FARs with fluorinated acetoacetates, followed by cyclization with hydrazines. ${ }^{42}, 43$ The limited availability of fluorinated acetoacetates encouraged us to turn towards other strategies. $\mathrm{NH}$-pyrazoles could also be obtained from the condensation of benzophenone-derived fluorinated azines with activated FARs, followed by cyclization under acidic conditions (Path b). However, a major drawback of this reaction was residual benzophenone, which proved difficult to remove even after several purifications. ${ }^{44,45}$ This method was then improved in terms of yield, reaction time and waste by the use of fluorinated $N$-benzyl imines (Path c). ${ }^{46,47}$
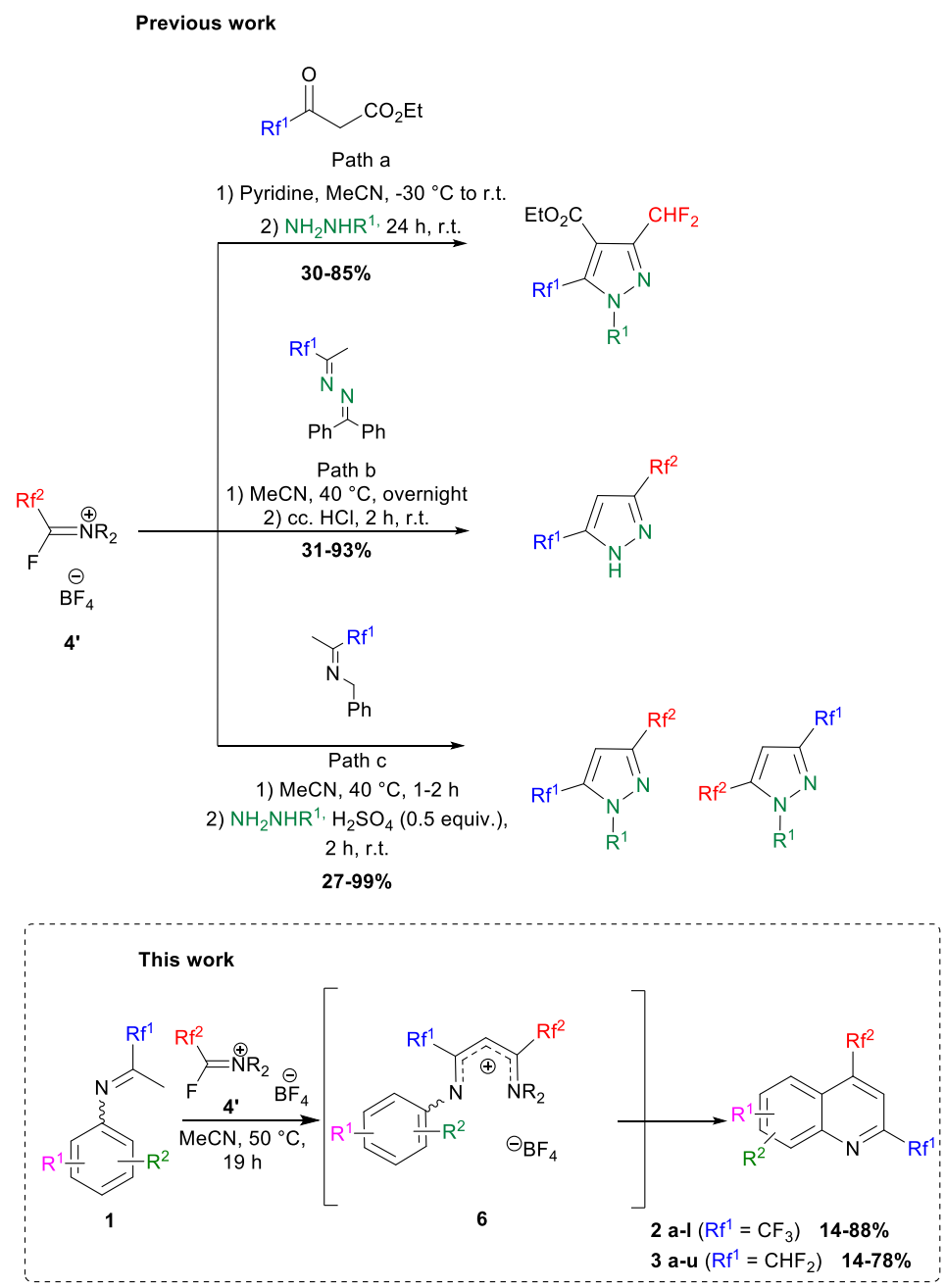

$\mathrm{R}: \mathrm{Me}, \mathrm{Et}$

$\mathrm{R}^{1}: \mathrm{H}, \mathrm{Me}, \mathrm{Ph}, \mathrm{tBu}$, aryl, benzyl, quinoline, ect

$\mathrm{R}^{2}: \mathrm{H}, \mathrm{F}, \mathrm{OMe}, \mathrm{Cl}, \mathrm{NMe}_{2}, \mathrm{OCF}_{3}, \mathrm{CF}_{3}, \mathrm{Ph}, \mathrm{SPh}$, fused phenol

$\mathrm{Rf}^{1}: \mathrm{CHF}_{2}, \mathrm{CH}_{2} \mathrm{~F}, \mathrm{CF}_{3}, \mathrm{C}_{2} \mathrm{~F}_{5}, \mathrm{CF}_{2} \mathrm{Cl}$

$\mathrm{Rf}^{2}: \mathrm{CHF}_{2}, \mathrm{CHFCl}_{2} \mathrm{CHFCF}_{3}, \mathrm{CHFOCF}_{3}$

Scheme 3: Previous work: Formation of fluorinated pyrazoles involving activated FARs: a) from fluorinated acetoacetates, b) from fluorinated azines and c) from fluorinated imines. This work: Formation of fluorinated quinoline derivatives involving activated FARs.

To further exploit the reactivity of FARs, we decided to use $\mathrm{N}$ aryl fluoroketimines. The reactions afforded bis-fluorinated quinolines $\mathbf{2}$ and $\mathbf{3}$ (Scheme 3). Such fluorinated quinolines are commonly prepared by first constructing the quinoline core (via the Skraup, Döbner-von Miller, Pfitzinger, Conrad-Limpach, Friedlander or Combes syntheses, to cite a few), $21,48,49$ mostly under harsh conditions, and sometimes with low yields. Then, fluorine might be introduced by means of fluorinating agents, Balz-Schiemann reaction, Halex process or HF-mediated. Nevertheless, such fluorinations often require multi-step reactions and particular equipement. ${ }^{31,50-59}$ Among the existing syntheses of fluorinated quinolines, none combines short synthetic pathway, mild reaction conditions, the possibility to introduce different fluorinated substituents in 
the same molecule and industrially suitable conditions. In particular, the synthesis of quinoline derivatives bearing two fluorinated groups in both positions 2 and 4 is scarcely described in the literature; only a few bis(trifluoromethylated)quinolines can be found. $57,58,60,61$ Some examples reported on the use of anilines and fluorinated $\beta$-diketones, which are difficultly accessible. The products were formed in low yields and their isolation proved to be tricky because of their volatility. Moreover, the cyclization step is usually performed under harsh conditions in polyphosphoric acid, which produces P-containing waste in large amounts, and the use of unsymmetrical $\beta$-diketones leads to mixtures of regioisomers. ${ }^{62-64}$ In the present work, we describe the access to quinolines substituted in positions 2 and 4 with different fluoroalkyl groups, under mild conditions and with complete regioselectivity.

\section{Results and discussion}

\section{Synthesis of quinoline derivatives}

Substituted anilines were condensed onto di- or trifluoroacetone $\mathbf{5}$ at room temperature in anhydrous dichloromethane (DCM) in presence of a desiccant (e.g. $4 \AA$ $\mathrm{MS}, \mathrm{MgSO}_{4}$ ) following the publication of Perrone et al. (Scheme 4). ${ }^{65}$ The desired fluorinated imines 1 were obtained in moderate to excellent yields (Tables 1 and 2). Ketimines obtained from 1,1-difluoroacetone gave better results than from trifluoroacetone. This can be explained by the weaker destabilization of the carbocation - due to the less intense electron-withdrawing effect of the $\mathrm{CHF}_{2}$ moiety compared to $\mathrm{CF}_{3}$ - during the hemi-aminal dehydration step. In some cases, the purity of the ketimine was low, either because of incomplete conversion - even after introduction of additional ketone or prolonged reaction time- or side reactions, e.g. self-condensation of the ketone. This can be avoided by performing the reaction at $0{ }^{\circ} \mathrm{C}$; however, under these conditions, the condensation between anilines and the ketones is either very slow or totally ineffective. Ketimines cannot be purified due to their sensitivity to moisture or silica. Nonetheless, the presence of side products in $\mathbf{1}$ did not seem to affect the next step. The stability of the ketimines is acceptable and they can be stored under argon for a few weeks without hydrolysis or degradation.
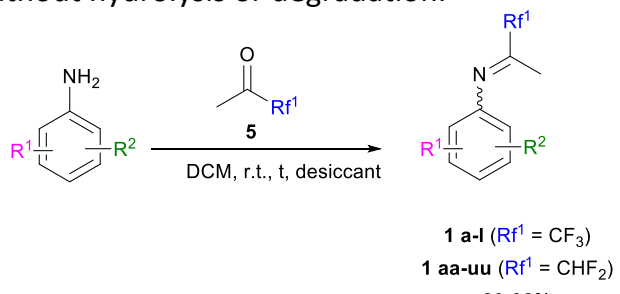

Scheme 4: Synthesis of fluorinated ketimines.

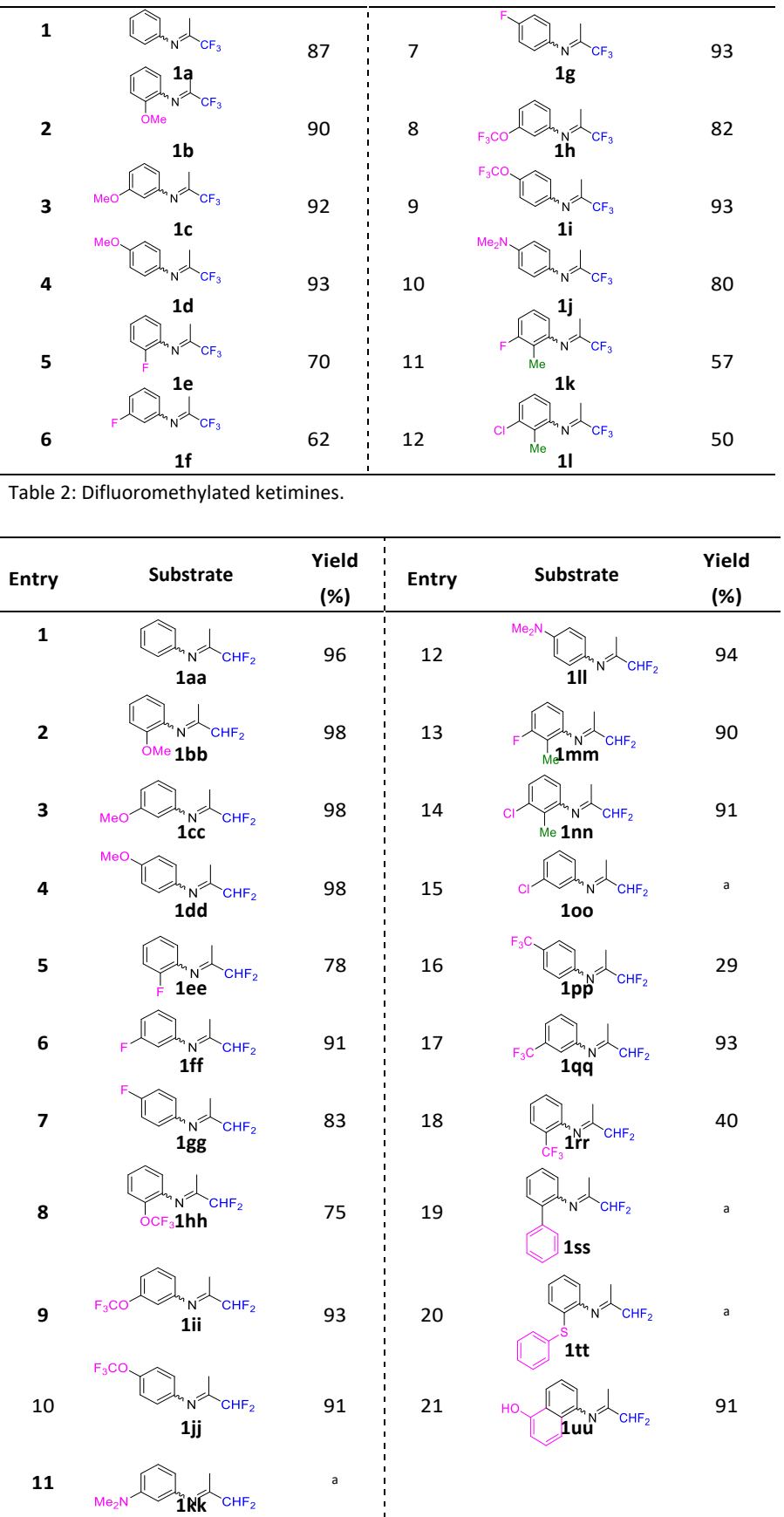

[a]: Mixture (SM, Impurities).

Next, ketimines 1 were reacted with activated FARs. In practice, FARs were treated beforehand with $\mathrm{BF}_{3} \bullet \mathrm{Et}_{2} \mathrm{O}$ in $\mathrm{MeCN}$ for $15 \mathrm{~min}$ and directly mixed with fluorinated imines. Upon nucleophilic attack of the enamine tautomer of 1 onto the iminium salt, the resulting vinamidinium intermediate 6 undergoes electrophilic aromatic substitution yielding quinolines $\mathbf{2}$ and 3, following a Combes-like rearrangement (Schemes 3, 5 and figure 2). ${ }^{66}$

Table 1: Trifluoromethylated ketimines.

\begin{tabular}{lcc:ccc}
\hline \multirow{2}{*}{ Entry } & \multirow{2}{*}{ Substrate } & Yield & Entry & Substrate & $\begin{array}{c}\text { Yield } \\
\end{array}$ \\
& $(\%)$ & & $(\%)$ \\
\hline
\end{tabular}




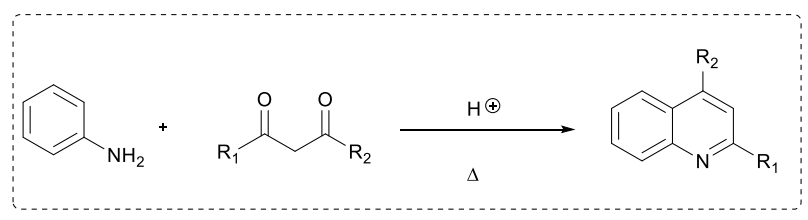

Scheme 5: Combes reaction.

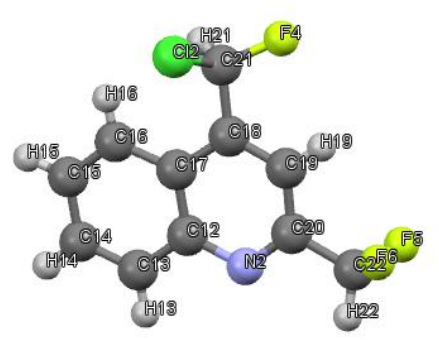

Figure 2: Crystal structure of 4-[chloro(fluoro)methyl]-2-(difluoromethyl)quinoline 3aii. ${ }^{67}$

In certain cases, the transformation was not complete and hydrolysis of the reaction mixture provided vinamides $6^{\prime}$, thus confirming the mechanistic pathway (Scheme 6).

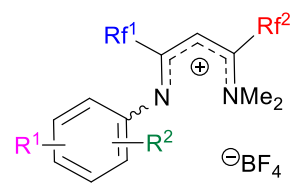

6

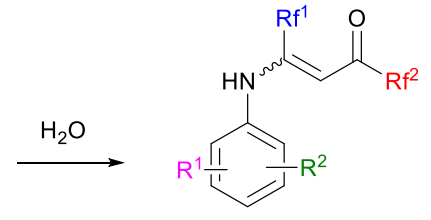

$6^{\prime}$
Scheme 6: Vinamidinium hydrolysis

The desired quinolines were obtained in moderate to good yields. All results are summarized below (Tables 3 and 4, figures 3 and 4).

In general, quinoline derivatives obtained from either the Yarovenko 4b or Ishikawa 4c reagents (Table 3: entries 2, 3, 10 and 11; Table 4: entries 2 and 9) were formed in lower yields than those obtained from the reaction with TFEDMA 4a. This confirms the higher reactivity of the latter reagent.

The synthesis of quinolines derived from $\mathrm{CF}_{3}$ substituted anilines (Table 4: entries 18-20) was performed, however the yields were not satisfying due to the high volatility of these compounds.

Starting from imines bearing a 3-substituent on the arene (Table 3: entries 5, 8 and 12; Table 4: entries 4, 7, 11, 13 and 19), only one regioisomer was observed after purification, namely 7-substituted quinolines. Indeed, the cyclisation of the vinamidinium intermediate 6 occurs at the less hindered position, i.e. para to the 3 -substituent of the imine.

Overall, the relative yields of quinolines in Tables 3 and 4 can be rationalized in part by an interplay of destabilizing and stabilizing effects operating on the vinamidinium intermediate on the one hand, and on the Wheland intermediate obtained during cyclisation on the other hand (Scheme 7).
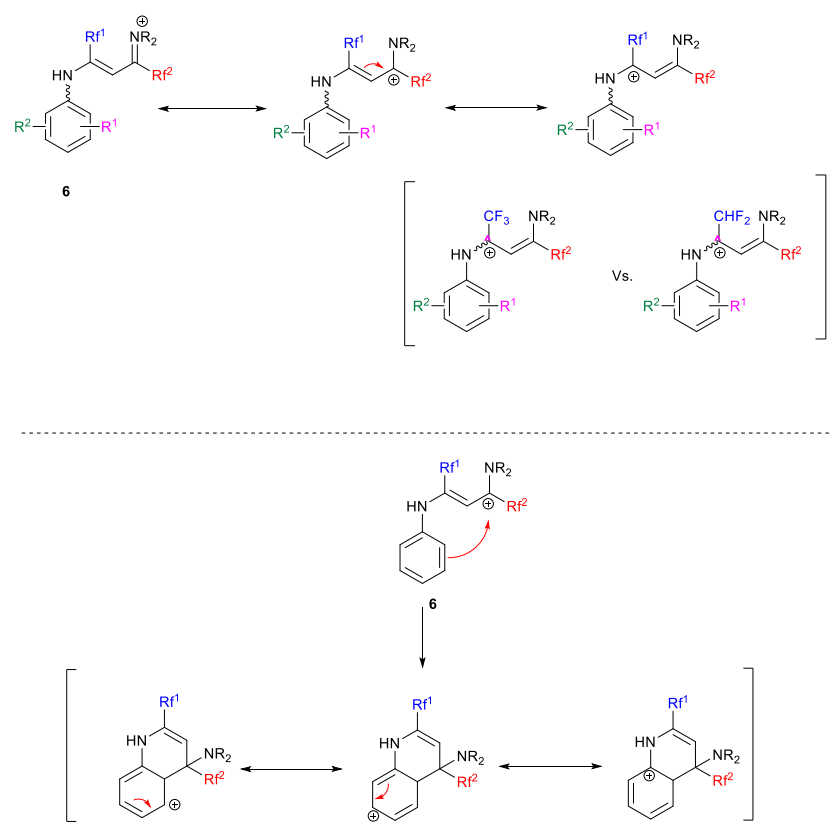

Scheme 7 : Stabilization/destabilization of intermediate 6 during the cyclization process.

Table 3: Synthesis of quinolines from 1,1,1-trifluoroacetone-derived imines 1a-I.

\begin{tabular}{|c|c|c|c|c|}
\hline Entry & Substrate & FAR & Product & Yield (\%) \\
\hline 1 & & $4 a$ & $2 a i$ & 62 \\
\hline 2 & $1 a$ & $4 b$ & 2aii & 57 \\
\hline 3 & & $4 c$ & 2aiii & 14 \\
\hline 4 & $1 \mathrm{~b}$ & & $2 b$ & 88 \\
\hline 5 & 1c & & $2 c$ & 64 \\
\hline 6 & 1d & $4 a$ & $2 d$ & 85 \\
\hline 7 & $1 e$ & & $2 e$ & 70 \\
\hline 8 & $1 f$ & & $2 f$ & 62 \\
\hline 9 & & $4 a$ & $2 \mathrm{gi}$ & 67 \\
\hline 10 & $1 \mathrm{~g}$ & $4 b$ & 2gii & $35^{b}$ \\
\hline 11 & & $4 c$ & 2giii & $1: 3^{c}$ \\
\hline 12 & $1 \mathrm{~h}$ & & $2 \mathrm{~h}$ & 36 \\
\hline 13 & $1 \mathbf{i}$ & & $2 i$ & 49 \\
\hline 14 & $1 \mathrm{j}$ & $4 a$ & $2 j$ & 37 \\
\hline 15 & $1 \mathrm{k}$ & & $2 k$ & 38 \\
\hline 16 & 11 & & 21 & 38 \\
\hline
\end{tabular}

[a]: Isolated yield except indicated otherwise; [b]:19F NMR yield by using fluorobenzene as internal standard. [c]: ${ }^{1} \mathrm{H}$ NMR ratio $\left(2: 6^{\prime}\right)$, not isolated. 

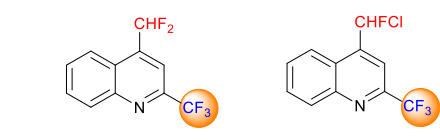<smiles>COc1ccc2c(CF)cc(-c3ccccc3)nc2c1</smiles>

2c<smiles>Fc1ccc2nc(-c3ccccc3)cc(C(F)F)c2c1</smiles>

2d<smiles>O=Cc1cc(CCl)nc2ccc(F)cc12</smiles>

2 gi

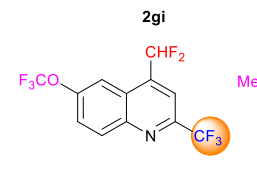

$2 \mathbf{i}$

2gii<smiles>CN(C)c1ccc2nc(-c3ccccc3)cc(C(F)F)c2c1</smiles>
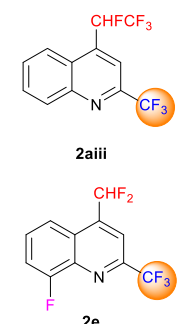

(2e)<smiles>O=[N+]([O-])c1c(F)ccc2c(C(F)F)cc(-c3ccccc3)nc12</smiles>
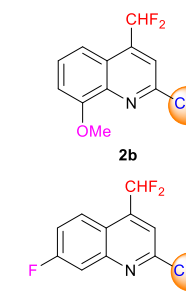

(2)

$\mathbf{2 h}$

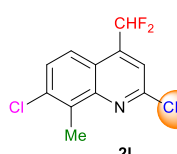

Figure 3: 4-(Difluoromethyl)-2-(trifluoromethyl)-substituted quinoline derivatives 2ai-I.

In the case where $\mathrm{Rf}^{1}$ is a $\mathrm{CF}_{3}$ group, the withdrawing effect of $\mathrm{CF}_{3}$ destabilizes the vinamidinium intermediate $\mathbf{6}$. The addition of mesomeric donor substituents on 6 might stabilize it. We indeed obtained similar or better results than starting from the corresponding $N$-phenyl substrate (Table 3 , entries $1,4-9$ ). A notable exception is the dimethylamino-substituted substrate (entry 14). When $\mathrm{Rf}^{1}$ is a $\mathrm{CF}_{3}$ group, the best results in terms of yields were obtained when mesomeric donor substituents are in position 2 and 4 (Table 3, entries 4 and 6 vs. entry 5, entries 7 and 9 vs. entry 8). The introduction of electron-withdrawing substituents destabilizes the vinamidinium even further and the cyclization is less efficient (Table 3 , entries $1,12-13$ ).

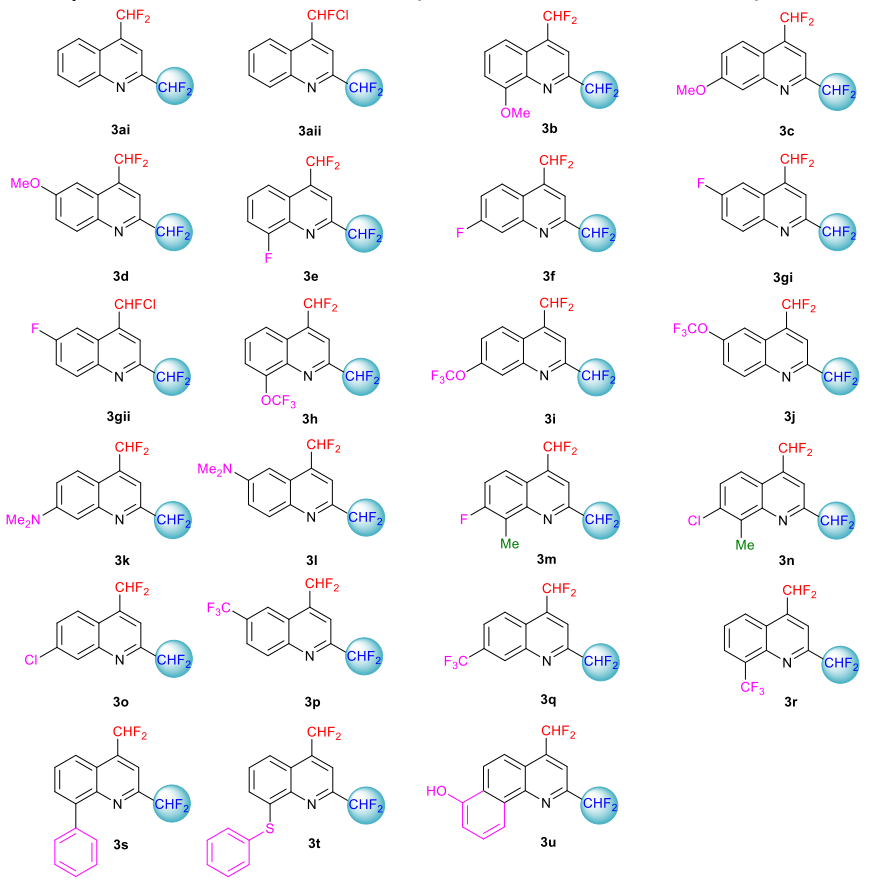

Figure 4: 2,4-Bis(difluoromethyl)-substituted quinoline derivatives 3ai-u.

Table 4: Synthesis of quinolines from 1,1-difluoroacetone-derived imines 1aa-uu.

\begin{tabular}{|c|c|c|c|c|}
\hline Entry & Substrate & FAR & Product & Yield (\%) \\
\hline 1 & \multirow{2}{*}{ 1aa } & $4 a$ & 3ai & 77 \\
\hline 2 & & $4 b$ & 3aii & 78 \\
\hline 3 & $1 \mathrm{bb}$ & \multirow{6}{*}{$4 a$} & $3 b$ & 72 \\
\hline 4 & $1 c c$ & & $3 c$ & 78 \\
\hline 5 & $1 \mathrm{dd}$ & & $3 d$ & 71 \\
\hline 6 & 1ee & & $3 e$ & $39(3: 1)^{b}$ \\
\hline 7 & $1 \mathrm{ff}$ & & $3 f$ & 70 \\
\hline 8 & \multirow{2}{*}{ 1gg } & & $3 g i$ & 39 \\
\hline 9 & & $4 b$ & 3gii & traces \\
\hline 10 & $1 \mathrm{hh}$ & \multirow{14}{*}{$4 a$} & $3 \mathrm{~h}$ & 39 \\
\hline 11 & $1 \mathrm{ii}$ & & $3 \mathbf{i}$ & 71 \\
\hline 12 & $1 \mathrm{jj}$ & & $3 \mathbf{j}$ & 58 \\
\hline 13 & $10 k$ & & $3 k$ & 15 \\
\hline 14 & 111 & & 31 & 14 \\
\hline 15 & $1 \mathrm{~mm}$ & & $3 m$ & 76 \\
\hline 16 & $1 \mathrm{nn}$ & & $3 n$ & 75 \\
\hline 17 & 100 & & 30 & 45 \\
\hline 18 & $1 p p$ & & $3 p$ & 23 \\
\hline 19 & $1 q q$ & & $3 q$ & 28 \\
\hline 20 & $1 \mathrm{rr}$ & & $3 r$ & traces \\
\hline 21 & 1ss & & $3 s$ & 28 \\
\hline 22 & $1 \mathrm{tt}$ & & $3 t$ & 52 \\
\hline 23 & 1uu & & $3 u$ & 51 \\
\hline
\end{tabular}

[a]: Isolated yield except indicated otherwise; [b]: ${ }^{1} \mathrm{H}$ NMR ratio $\left(\mathbf{3}: \mathbf{6}^{\prime}\right)$, not isolated.

When $\mathrm{Rf}^{1}$ is a $\mathrm{CHF}_{2}$, the vinamidinium intermediate 6 is less destabilized. Cyclization would therefore be facilitated by mesomeric donor substituents (Table 4, entries 1, 3-5, 7 vs. 10, $12,18-20)$. As expected quinoline derivatives which possess mesomeric donor substituents in position 7 gave the best results. Indeed the carbocation is stabilized during the cyclization process (Table 4, entry 4 vs. entries 3 and 5, entry 7 vs. entries 6 and 8 ). However, once again the dimethylamino group fails to obey this rule (entry 13), and a $3-\mathrm{OCF}_{3}$ substituent leads to a surprisingly high yield of $71 \%$ (entry 11 ).

Clearly, other subtle effects have yet to be invoked to account for the observed reactivities. In general, higher yields are obtained for 7-substituted quinolines compared to 6- or 8substituted ones with $\mathrm{Rf}^{1}=\mathrm{CHF}_{2}$ (Table 4 , entry 4 vs. entries 3 and 5; entry 7 vs. entries 6 and 8), while the opposite applies when $\mathrm{Rf}^{1}$ is a $\mathrm{CF}_{3}$ (Table 3 , entry 5 vs. entries 4 and 6 ; entry 8 vs. entries 7 and 9). 


\section{Introduction of new functionalities in position 8 by oxidation}

Commercially available quinolines named Quinmerac and Quinclorac, developed by BASF, have shown some activities as selective herbicides and are used on cereals to control weeds growth. ${ }^{68,69}$ The particularity of these molecules is that they bear a carboxylic acid function in position 8 (Scheme 8).<smiles>O=C(O)c1c(Cl)ccc2cc(Cl)cnc12</smiles>

Quinclorac<smiles>Cc1cnc2c(C(=O)O)c(Cl)ccc2c1</smiles>

Quinmerac
Scheme 8: Molecular structures of Quinclorac and Quinmerac.

Thus, we were interested in the introduction of a carboxylic acid function in the same position on our fluorinated quinoline derivatives. Bis-substituted quinolines $\mathbf{3 n}$ and $\mathbf{3 m}$ were chosen as precursors for the synthesis of bis(fluoroalkyl) analogues of Quinmerac and Quinclorac in order to test their potential activity as phytosanitary ingredients.

We first attempted to oxidize the methyl group of quinolines $\mathbf{3} \mathbf{m}-\mathbf{n}$ under photochemical conditions, following the procedure of Togo et al., which should afford the desired carboxylic acids in presence of oxone and potassium bromide in a mixture of water and dichloromethane. ${ }^{70}$ Nevertheless, the reaction did not allow the access to the desired compounds but to the 8-(bromomethyl)-2,4bis(difluoromethyl)-7-halo-quinolines $7(X=F)$ and $8(X=C l)$ (Scheme 9). Alternately, the oxidation conditions of Sudalai et al. (aqueous sulfuric acid, sodium periodate and lithium bromide in the absence of solvent $)^{71}$ provided the same 8 (bromomethyl)quinoline derivatives 7 and 8 in 50 and 55\% yields, respectively. Although it requires a slightly longer reaction time, the photochemical procedure was preferred as it gave better results and is also easier to carry out.<smiles>[X]c1ccc2c(C(F)F)cc(C(F)F)nc2c1[N+](=O)[O-]</smiles>

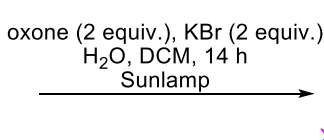

$3 \mathrm{~m}, \mathrm{X}=\mathrm{F}$ $3 n, X=C l$

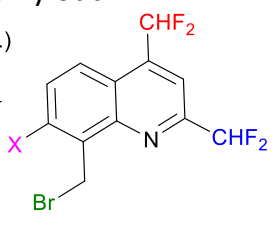

$7, X=F(63 \%)$ $8, \mathrm{X}=\mathrm{Cl}(68 \%)$
Scheme 9: Methyl group oxidation by photochemistry

In 1984, H. Hagen and coworkers described the oxidation of a bromomethyl quinoline derivative into its corresponding carboxylic acid (Quinmerac) thanks to a mixture of concentrated acids at $110{ }^{\circ} \mathrm{C} .69$ Following this procedure, 7chloro-2,4-bis(difluoromethyl)quinoline-8-carboxylic acid $\mathbf{9}$ was obtained in $64 \%$ yield (Scheme 10 ).

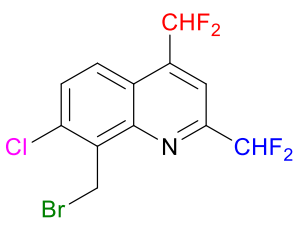

8

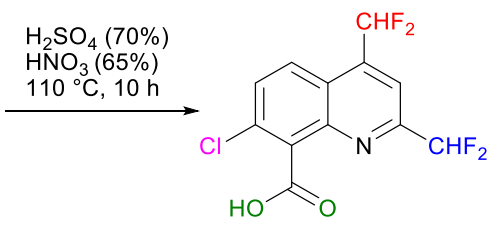

Scheme 10: Oxidation of 8-(bromomethyl)-7-chloro-2,4-bis(difluoromethyl)quinoline 8 into its corresponding carboxylic acid 9 .

The structure of the desired compound was confirmed by $X$ Ray diffraction crystallography, which allowed us to observe the presence of a reciprocal intermolecular hydrogen bonding between the hydroxyl group of one molecule and the nitrogen of the other (Figure 5).

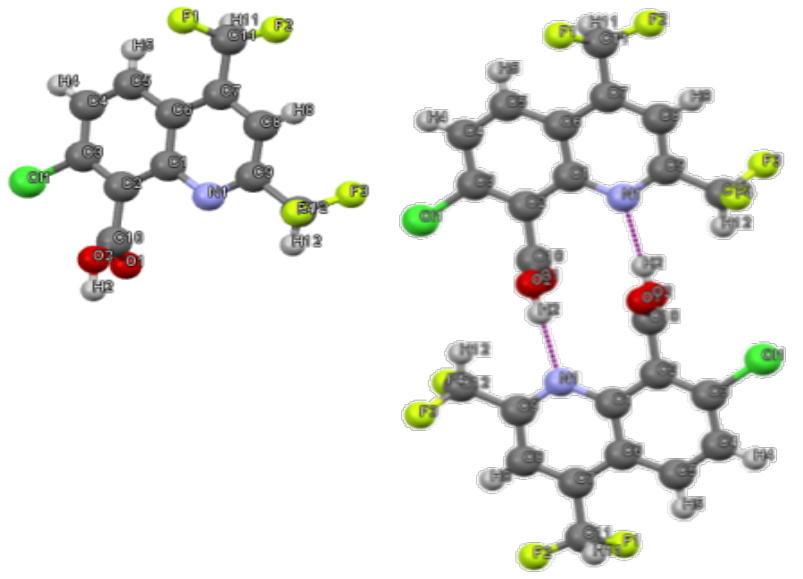

Figure 5: Single crystal X-ray diffraction structure of $9 .^{67}$

In parallel, we attempted the transformation of the methyl in position 8 into a nitrile according to Togo et al., employing aq. $\mathrm{HBr}$, aq. $\mathrm{H}_{2} \mathrm{O}_{2}$ in $\mathrm{CCl}_{4}$ followed by the addition of aq. $\mathrm{NH}_{3}$ and $\mathrm{I}_{2}$ in $\mathrm{MeCN} .{ }^{72}$ After 14 hours of reaction under sunlamp irradiation at $60{ }^{\circ} \mathrm{C}, 29 \%$ of the desired 7-chloro-2,4bis(difluoromethyl)quinoline-8-carbonitrile 10 was obtained. After purification, traces of 8-(dibromomethyl)-7-chloro-2,4bis(difluoromethyl)quinoline $\mathbf{1 2}$ and $10 \%$ of the tris(quinoline8-ylmethyl)amine $\mathbf{1 1}$ were isolated (Scheme 11).

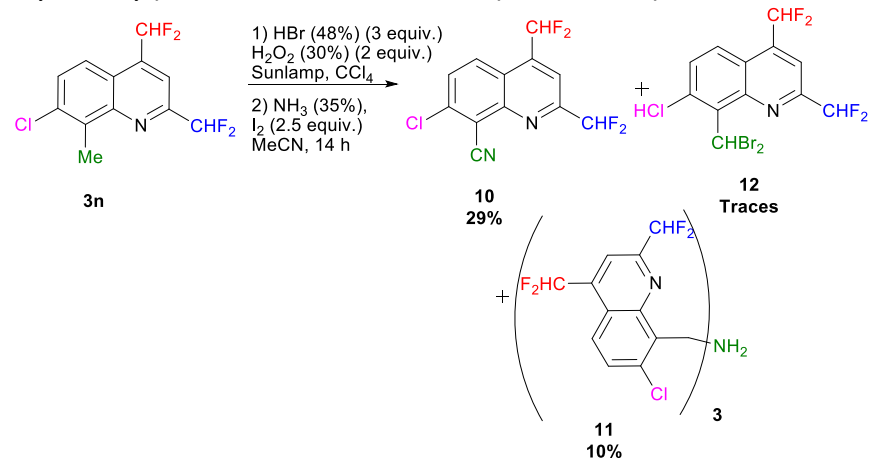

Scheme 11: One-pot transformation of 8-methylquinoline $3 n$ into the corresponding quinoline-8-carbonitrile 10 
Then, oxidation of the 2,4-bis(difluoromethyl)-8(phenylsulfanyl)quinoline 3t was performed following the procedure of Kim et al. using iron(III) chloride and periodic acid. ${ }^{73}$ The desired sulfoxide $\mathbf{1 3}$ was obtained in $\mathbf{7 9 \%}$ yield in racemic form. The diastereotopic fluorine atoms observed in ${ }^{19} \mathrm{~F}$ NMR confirm the presence of the stereocenter on the molecule (Scheme 12).<smiles>FC(F)c1cc(C(F)F)c2cccc(Sc3ccccc3)c2n1</smiles>

$3 t$

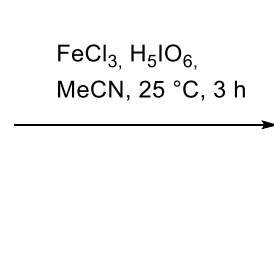

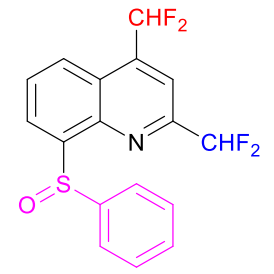

13
Scheme 12: Oxidation of $\mathbf{3 t}$ into sulfoxide 13

Several other oxidation protocols were attempted in order to functionalize the position 8 of quinolines 7 and 8 but all failed. The low electron-density of these bis(fluoroalkyl)quinolines render them difficult to oxidize, as attested by the need of really harsh conditions, even at high temperature, to afford the desired compounds in moderate yields only.

\section{Comparison between the use of FARs and carboxylic anhydrides or oxalate derivatives in the synthesis of quinolines}

Furthermore, we studied the reaction of ketimine 1aa with carboxylic anhydrides and acyl chloride. Indeed, various anhydrides or acyl chlorides are commercially available and could enlarge the scope of the approach. The purpose was to study the possibility to access to new quinoline derivatives by using this abundant starting material.

Our first attempt used acetic anhydride (1 equiv.) in either dichloromethane or acetonitrile, from -5 to $50{ }^{\circ} \mathrm{C}$, in presence of bases such as pyridine or DIPEA ( 1.5 to 2 equiv.) or without. At the end of the reaction, the $\mathrm{N}$-phenylacetamide 14 was always obtained after $N$-acylation and hydrolysis of the ketimine 1aa in 22 to $91 \%$ yields (Scheme 13).

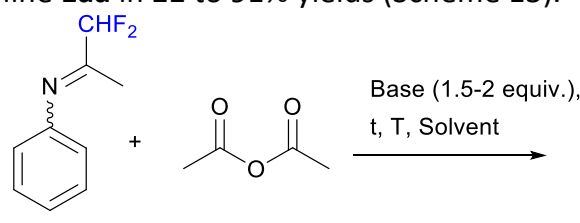

1 aa

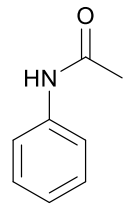

14
Scheme 13: Reaction between ketimine 1aa and acetic anhydride.

When ketimine 1aa was reacted with ethyl oxalyl monochloride (1 equiv.) under the same series of conditions as for acetic anhydride, the corresponding ethyl-2-oxo-2(phenylamino)acetate 15 was obtained in 65 to $87 \%$ yields (Scheme 14). However, when 1aa was dissolved in acetonitrile in presence of pyridine ( 1.5 equiv.) at room temperature, a small amount of vinamide $\mathbf{1 6}$ was obtained as a minor fraction in mixture with unidentified impurities.

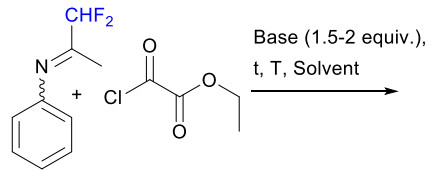

1 aa

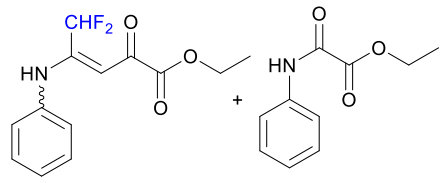

16

15
Scheme 14: Reaction between ketimine 1aa and ethyl oxalyl monochloride.

In a second part, we decided to study the reactivity of ketimine 1aa in presence of fluorinated acetic anhydrides (1 equiv.). First, when trifluoroacetic anhydride was used, the corresponding 2,2,2,-trifluoro- $N$-phenylacetamide 17 was obtained. In dichloromethane in presence of pyridine (1.5 equiv.) at -5 to $50{ }^{\circ} \mathrm{C}$ and in acetonitrile in presence of DIPEA (2 equiv.) at room temperature, only the compound $\mathbf{1 7}$ was formed in respectively 65 and 35\% yield. However, in acetonitrile in presence of pyridine (1.5 equiv.) or without base, at room temperature or at $50{ }^{\circ} \mathrm{C}$, the desired vinamide 18 was obtained in 20 to $49 \%$ yields (Scheme 15).

Next, difluoroacetic anhydride was reacted with ketimine 1aa under the same reaction conditions (Scheme 15). In dichloromethane in presence of pyridine ( 1.5 equiv.) at -5 to $50{ }^{\circ} \mathrm{C}, 45 \%$ yield of 2,2,-difluoro- $N$-phenylacetamide 19 was obtained. Vinamide $\mathbf{2 0}$ was observed in a small amount in mixture with degradation products and isolated in $29 \%$ yield after purification by column chromatography. DIPEA, used instead of pyridine also led to the formation of amide $\mathbf{1 9}$ as a major product in $47 \%$ yield and only traces of vinamide $\mathbf{2 0}$ were isolated.

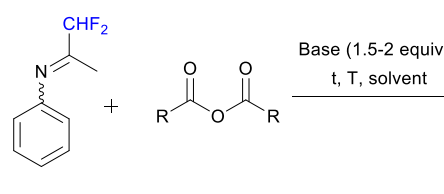

1 aa

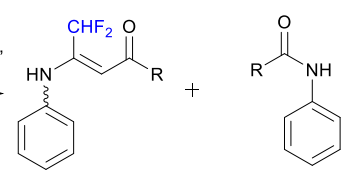

$\mathrm{R}=\mathrm{CF}_{3}: 20-49 \%(18), \quad \mathrm{R}=\mathrm{CF}_{3}: 15-65 \%(17)$, $\mathrm{R}=\mathrm{CHF}_{2}:$ traces-29\% (20) $\mathrm{R}=\mathrm{CHF}_{2}: 45-98 \%(19)$

Scheme 15: Reaction between ketimine 1aa and fluorinated anhydrides.
The vinamides $\mathbf{1 8}$ and $\mathbf{2 0}$ were finally involved in a cyclization reaction in presence of concentrated $\mathrm{H}_{2} \mathrm{SO}_{4}$ (10 equiv.) at $50{ }^{\circ} \mathrm{C}$ for $2 \mathrm{~h}$. The corresponding quinoline derivatives 3 ai and $\mathbf{2 3}$ were obtained in 64 and 90\% yield, respectively (Scheme 16).

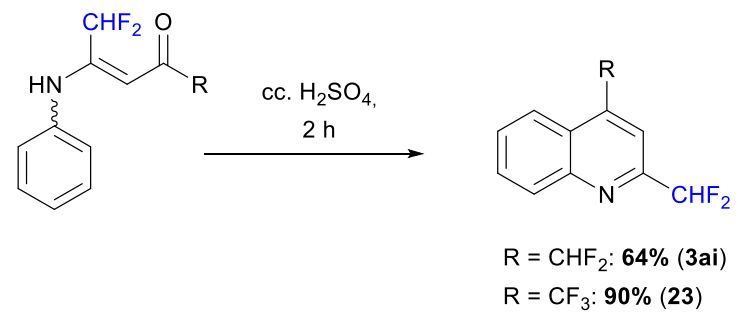

Scheme 16: Cyclization reaction of vinamide. 
These results confirm the superiority of FARs in the synthesis of quinoline derivatives in one step with overall good yields. Although the use of anhydrides or acyl chlorides was a synthetic asset, they have not allowed to access the desired quinoline derivatives in one step, as they afforded first the intermediate vinamides which had to be converted into the quinolines under harsh conditions.

\section{Conclusions}

In summary, this work describes a new and efficient way to access quinolines bearing two different or identical fluoroalkyl groups in positions 2 and 4. FARs proved to be highly useful tools in the synthesis of these fluorinated heterocycles and afforded them in moderate to high yield (14-88\%) under mild conditions.

\section{Experimental section}

Materials and methods.

All reactions were performed in flame-dried glassware using Schlenk techniques. Liquids and solutions were transferred with syringes. Air- and moisture- sensitive materials were stored and handled under an atmosphere of argon. Solvents were purified and dried following standard procedures: Dichloromethane (DCM) and Tetrahydrofuran (THF) were distilled from $\mathrm{CaH}_{2}$ or sodium + benzophenone prior to use.

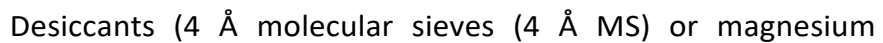
sulphate $\left(\mathrm{MgSO}_{4}\right)$ were previously activated in an oven. Technical grade solvents for extraction and chromatography (cyclohexane, dichloromethane, $n$-pentane, ether, toluene, and ethyl acetate) were used without purification. Starting materials, if commercial, were purchased from standard suppliers (Sigma-Aldrich, Acros, Alfa Aesar and Apollo scientific) and used as such, provided that adequate checks (NMR) had confirmed the claimed purity. Analytical thin-layer chromatography (TLC) was carried out on $0.25 \mathrm{~mm}$ Merck silica-gel (60-F254). Flash column chromatography was performed on silica gel 60 (40-63 $\mu \mathrm{m}, 230-400$ mesh, ASTM) by Merck using the indicated solvents. ${ }^{1} \mathrm{H},{ }^{13} \mathrm{C}$, and ${ }^{19} \mathrm{~F}-\mathrm{NMR}$ spectra were recorded in $\mathrm{CDCl}_{3}$ on Bruker AV 400 instruments $\left({ }^{1} \mathrm{H}: 400 \mathrm{MHz},{ }^{19} \mathrm{~F}: 376 \mathrm{MHz},{ }^{13} \mathrm{C}: 101 \mathrm{MHz}\right)$. Chemical shifts are reported in parts per million (ppm) and are referenced to the residual solvent resonance as the internal standard (chloroform $\left(\delta\left[{ }^{1} \mathrm{H}\right]=7.26\right.$ and accordingly $\delta\left[{ }^{13} \mathrm{C}\right]=77.16$ ppm). Data are reported as follows: chemical shift, multiplicity (br $\mathrm{s}=$ broad singlet, $\mathrm{s}=$ singlet, $\mathrm{d}=$ doublet, $\mathrm{t}=$ triplet, $\mathrm{q}=$ quartet, $\mathrm{m}=$ multiplet, $\mathrm{td}=$ triplet of doublets, $\mathrm{dd}=$ doublet of doublets), coupling constant $(\mathrm{Hz})$ and integration. The spectra were processed with the program MestReNova (Version 6.0.25475). Melting points (MP) were determined for crystalline compounds with a Büchi Melting Point Apparatus M-560 and are not corrected. IR spectra were measured with a Perkin Elmer Spectrum UATR two (diamond detection). HRMS analysis (measurement accuracy $\leq 15 \mathrm{ppm}$ ) and EA were performed by the analytical facility at the University of
Strasbourg. Crystal X-ray diffraction analysis was carried out by the Radiocrystallography Service of the University of Strasbourg.

\section{General procedure for the synthesis of ketimines derivatives 1}

Under argon atmosphere, an excess of cold perfluoroacetone ( 2 equiv.) was added to aniline derivatives (1 equiv.) in anhydrous DCM ( $2 \mathrm{~mL} / 1 \mathrm{mmol})$ in presence of desiccant (e.g. (4 $\AA \mathrm{MS}$ ) or $\mathrm{MgSO}_{4}$ ). Then the reaction mixture was stirred for the indicated time at room temperature. The desiccant was then filtered off on celite and washed with ether. The filtrate was concentrated under reduced pressure to provide the desired product. Ketimines cannot be purified due to their sensitivity to moisture or silica so the compound was used without any further purification.

$\mathbf{N}$-(1,1,1-Trifluoropropan-2-ylidene)aniline $\mathbf{1 a}$. The product was prepared according to the general procedure and starting from 1,1,1-trifluoroacetone (2 equiv., $1.92 \mathrm{~mL}, 21.4 \mathrm{mmol}$ ) and aniline ( 1 equiv., $0.98 \mathrm{~mL}, 10.7 \mathrm{mmol}$ ) in presence of (4 Å MS). The reaction mixture was stirred for $3 \mathrm{~h}$ at room temperature. $\mathrm{N}$-(1,1,1-Trifluoropropan-2-ylidene)aniline 1 a was provided as a brown oil (1.74 g, 87\%, estimated). ${ }^{1} \mathrm{H} N M R \delta H=7.38(2 \mathrm{H}, \mathrm{t}$, $\left.{ }^{3} J_{H-H}=7.9 \mathrm{~Hz}, \mathrm{C}(3,5) \mathrm{H}\right), 7.18\left(1 \mathrm{H}, \mathrm{t},{ }^{3} J_{\mathrm{H}-\mathrm{H}}=7.5 \mathrm{~Hz}, \mathrm{C}(4) \mathrm{H}\right), 6.82-$ $6.74(2 \mathrm{H}, \mathrm{m}, \mathrm{C}(2,6) \mathrm{H}), 2.02(3 \mathrm{H}, \mathrm{s}, \mathrm{Me}) \mathrm{ppm} .{ }^{19} \mathrm{~F} \mathrm{NMR} \delta \mathrm{F}=-$ $74.68\left(3 \mathrm{~F}, \mathrm{~s}, \mathrm{CF}_{3}\right) \mathrm{ppm} .{ }^{13} \mathrm{C} \mathrm{NMR} \delta \mathrm{C}=157.41\left(\mathrm{q},{ }^{2} J_{\mathrm{C}-\mathrm{F}}=33.9 \mathrm{~Hz}\right.$, $\mathrm{CCF}_{3}$ ), 147.72 (s, C-1), 129.33 (2 C, s, C-3,5), 125.29 (s, C-4), $119.87\left(\mathrm{q},{ }^{1} J_{C-F}=278.4 \mathrm{~Hz}, \mathrm{CF}_{3}\right) 118.87(2 \mathrm{C}, \mathrm{s}, \mathrm{C}-2,6), 14.50$ (s, $\mathrm{Me}) \mathrm{ppm}$.

2-Methoxy-N-(1,1,1-trifluoropropan-2-ylidene)aniline 1b. The product was prepared according to the general procedure and starting from 1,1,1-trifluoroacetone (2 equiv., $1.46 \mathrm{~mL}, 16.2$ $\mathrm{mmol}$ ) and 2-methoxyaniline (1 equiv., $0.92 \mathrm{~mL}, 8.12 \mathrm{mmol}$ ) in presence of ( $4 \AA \mathrm{MS})$. The reaction mixture was stirred for $21 \mathrm{~h}$ at room temperature. 2-Methoxy- $N$-(1,1,1-trifluoropropan-2ylidene)aniline $\mathbf{1 b}$ was provided as a brown oil (1.59 $\mathrm{g}, 90 \%)$. ${ }^{1} \mathrm{H}$ NMR $\delta \mathrm{H}=7.16-7.12(1 \mathrm{H}, \mathrm{m}, \mathrm{C}(4) \mathrm{H}), 7.00-6.90(2 \mathrm{H}, \mathrm{m}$, $\mathrm{C}(5,6) \mathrm{H}), 6.78-6.75(1 \mathrm{H}, \mathrm{m}, \mathrm{C}(3) \mathrm{H}), 3.78(3 \mathrm{H}, \mathrm{s}, \mathrm{OMe}), 1.95$ (3 $\mathrm{H}, \mathrm{s}, \mathrm{Me}) \mathrm{ppm} .{ }^{19} \mathrm{~F} N M R \delta \mathrm{F}=-74.33--74.42\left(3 \mathrm{~F}, \mathrm{~m}, \mathrm{CF}_{3}\right) \mathrm{ppm}$. ${ }^{13} \mathrm{C} N M R \delta C=158.86\left(q,{ }^{2} J_{C-F}=33.7 \mathrm{~Hz}, C_{C F}\right), 148.19(\mathrm{~s}, \mathrm{C}-2)$, 136.43 (s, C-1), 126.15 (s, C-4), 120.90 (s, C-5), 120.14 (s, C-3), $119.77\left(\mathrm{q},{ }^{1} J_{C-F}=278.4 \mathrm{~Hz}, \mathrm{CF}_{3}\right), 111.78(\mathrm{~s}, \mathrm{C}-6), 55.51$ (s, OMe), $14.90(\mathrm{~s}, \mathrm{Me}) \mathrm{ppm}$. HRMS (ESI +) for $\mathrm{C}_{10} \mathrm{H}_{11} \mathrm{~F}_{3} \mathrm{NO}[\mathrm{M}+\mathrm{H}]$ : calcd 218.0787, found 218.0773 .

3-Methoxy-N-(1,1,1-trifluoropropan-2-ylidene)aniline 1c. The product was prepared according to the general procedure and starting from 1,1,1-trifluoroacetone (2 equiv., $1.47 \mathrm{~mL}, 16.4$ $\mathrm{mmol}$ ) and 3-methoxyaniline (1 equiv., $0.91 \mathrm{~mL}, 8.12 \mathrm{mmol}$ ) in presence of ( $4 \AA \mathrm{MS})$. The reaction mixture was stirred for $24 \mathrm{~h}$ at room temperature. 3-Methoxy- $N$-(1,1,1-trifluoropropan-2ylidene)aniline 1c was provided as a brown oil (1.62 g, 92\%, estimated). ${ }^{1} \mathrm{H} N M R \delta \mathrm{H}=7.35-7.22(1 \mathrm{H}, \mathrm{m}, \mathrm{C}(5) \mathrm{H}), 6.79-$ $6.70(1 \mathrm{H}, \mathrm{m}, \mathrm{C}(4) \mathrm{H}), 6.44-6.32(2 \mathrm{H}, \mathrm{m}, \mathrm{C}(2,6) \mathrm{H}), 3.79(3 \mathrm{H}, \mathrm{s}$, $\mathrm{OMe}), 2.05(3 \mathrm{H}, \mathrm{s}, \mathrm{Me}) \mathrm{ppm} .{ }^{19} \mathrm{~F} \mathrm{NMR} \delta \mathrm{F}=-74.81\left(3 \mathrm{~F}, \mathrm{~s}, \mathrm{CF}_{3}\right)$, $74.91\left(3 \mathrm{~F}, \mathrm{~s}, \mathrm{CF}_{3}\right.$ ) ppm. ${ }^{13} \mathrm{C}$ NMR $\delta \mathrm{C}=160.50(\mathrm{~s}, \mathrm{C}-3), 157.56$ (q, $\left.{ }^{2} J_{C-F}=33.9 \mathrm{~Hz}, C C_{3}\right), 148.95(\mathrm{~s}, \mathrm{C}-1), 130.08(\mathrm{~s}, \mathrm{C}-5), 119.78$ (q, $\left.{ }^{1} J_{C-F}=278.2 \mathrm{~Hz}, C_{3}\right), 110.60(2 \mathrm{C}, \mathrm{s}, \mathrm{C}-4,6), 104.49(\mathrm{~s}, \mathrm{C}-2)$, 
54.91 (s, OMe), 14.01 (s, Me) ppm. HRMS (ESI +) for $\mathrm{C}_{10} \mathrm{H}_{11} \mathrm{~F}_{3} \mathrm{NO}[\mathrm{M}+\mathrm{H}]$ : calcd 218.0787, found 218.0818.

4-Methoxy- $N$-(1,1,1-trifluoropropan-2-ylidene)aniline $1 \mathrm{~d}$. The product was prepared according to the general procedure and starting from 1,1,1-trifluoroacetone (2 equiv., $1.47 \mathrm{~mL}, 16.4$ $\mathrm{mmol}$ ) and 4-methoxyaniline (1 equiv., $1.01 \mathrm{~g}, 8.2 \mathrm{mmol}$ ) in presence of ( $4 \AA \mathrm{MS})$. The reaction mixture was stirred for $20 \mathrm{~h}$ at room temperature. 4-Methoxy- $N$-(1,1,1-trifluoropropan-2ylidene)aniline 1d was provided as a brown oil (1.65 $\mathrm{g}, 93 \%)$. ${ }^{1} \mathrm{H} N M R \delta H=6.86\left(2 \mathrm{H}, \mathrm{m}, \mathbf{A A}^{\prime}, \mathrm{C}(2,6) \mathrm{H}\right), 6.73\left(2 \mathrm{H}, \mathrm{m}, \mathbf{B B}^{\prime}\right.$, $\mathrm{C}(3,5) \mathrm{H}), 3.75-3.71(3 \mathrm{H}, \mathrm{m}, \mathrm{OMe}), 2.03-2.00(3 \mathrm{H}, \mathrm{m}, \mathrm{Me})$ ppm. ${ }^{19} \mathrm{~F} N M R \delta F=-74.58--74.63\left(3 \mathrm{~F}, \mathrm{~m}, \mathrm{CF}_{3}\right) \mathrm{ppm} .{ }^{13} \mathrm{C} \mathrm{NMR}$ $\delta C=157.51(\mathrm{~s}, \mathrm{C}-4), 156.53\left(\mathrm{q},{ }^{2} J_{C-F}=33.5 \mathrm{~Hz}, C_{C F}\right), 140.36(\mathrm{~s}$, C-1), 120.85 (2 C, s, C-3,5), 119.96 (q, ${ }^{1} J_{C-F}=278.1 \mathrm{~Hz}, \mathrm{CF}_{3}$ ), 114.31 (2 C, s, C-2,6), 55.09 (s, OMe), 13.99 (s, Me) ppm. HRMS $\left(\mathrm{ESI}+\right.$ ) for $\mathrm{C}_{10} \mathrm{H}_{11} \mathrm{~F}_{3} \mathrm{NO}[\mathrm{M}+\mathrm{H}]$ : calcd 218.0787, found 218.0815.

2-Fluoro-N-(1,1,1-trifluoropropan-2-ylidene)aniline 1e. The product was prepared according to the general procedure and starting from 1,1,1-trifluoroacetone (2 equiv., $3.23 \mathrm{~mL}, 36$ $\mathrm{mmol}$ ) and 2-fluoroaniline (1 equiv., $1.74 \mathrm{~mL}, 18 \mathrm{mmol}$ ) in presence of ( $4 \AA \mathrm{MS})$. The reaction mixture was stirred for $16 \mathrm{~h}$ at room temperature. 2-Fluoro- $N$-(1,1,1-trifluoropropan-2ylidene)aniline $1 \mathrm{e}$ was provided as a brown oil $(2.58 \mathrm{~g}, 70 \%)$. ${ }^{1} \mathrm{H} N M R \delta H=7.21-7.07(3 \mathrm{H}, \mathrm{m}, \mathrm{C}(4,5,6) \mathrm{H}), 6.93-6.86(1 \mathrm{H}$, $\mathrm{m}, \mathrm{C}(3) \mathrm{H}), 2.02(3 \mathrm{H}, \mathrm{s}, \mathrm{Me}) \mathrm{ppm} .{ }^{19} \mathrm{~F} N M R \delta \mathrm{F}=-74.65(3 \mathrm{~F}, \mathrm{~s}$, $\left.\mathrm{CF}_{3}\right),-126.51--126.58(1 \mathrm{~F}, \mathrm{~m}, \mathrm{~F}) \mathrm{ppm} .{ }^{13} \mathrm{C}$ NMR $\delta \mathrm{C}=160.40$ (qd, $\left.{ }^{2} J_{C-F}=34.1,{ }^{4} J_{C-F}=0.5 \mathrm{~Hz}, C C F_{3}\right), 151.01\left(\mathrm{~d},{ }^{1} J_{C-F}=246.3 \mathrm{~Hz}\right.$, C-2), $134.91\left(\mathrm{~d},{ }^{2} J_{C-F}=12.8 \mathrm{~Hz}, \mathrm{C}-3\right), 126.57\left(\mathrm{~d},{ }^{3} J_{C-F}=7.3 \mathrm{~Hz}, \mathrm{C}-\right.$ 4), $124.59\left(\mathrm{~d},{ }^{4} J_{C-F}=3.8 \mathrm{~Hz}, \mathrm{C}-5\right), 121.68\left(\mathrm{~d},{ }^{3} J_{C-F}=1.1 \mathrm{~Hz}, \mathrm{C}-6\right)$, $119.53\left(\mathrm{q},{ }^{1} J_{C-F}=278.2 \mathrm{~Hz}, \mathrm{CF}_{3}\right), 116.18\left(\mathrm{~d},{ }^{2} J_{C-F}=19.7 \mathrm{~Hz}, \mathrm{C}-3\right)$, $15.01(\mathrm{~s}, \mathrm{Me})$ ppm. HRMS $\left(\mathrm{ESI}+\right.$ ) for $\mathrm{C}_{9} \mathrm{H}_{8} \mathrm{~F}_{4} \mathrm{~N}[\mathrm{M}+\mathrm{H}]$ : calcd 206.0587, found 206.0590.

3-Fluoro- $\mathbf{N - ( 1 , 1 , 1 - t r i f l u o r o p r o p a n - 2 - y l i d e n e ) a n i l i n e ~ 1 f . ~ T h e ~}$ product was prepared according to the general procedure and starting from 1,1,1-trifluoroacetone (2 equiv., $1.61 \mathrm{~mL}, 18$ $\mathrm{mmol}$ ) and 3-fluoroaniline (1 equiv., $0.87 \mathrm{~mL}, 9 \mathrm{mmol}$ ) in presence of ( $4 \AA \mathrm{MS})$. The reaction mixture was stirred for $48 \mathrm{~h}$ at room temperature. 3-Fluoro- $N$-(1,1,1-trifluoropropan-2ylidene)aniline $1 \mathrm{f}$ was provided as a brown oil $(1.14 \mathrm{~g}, 62 \%) .{ }^{1} \mathrm{H}$ $\mathrm{NMR} \delta \mathrm{H}=7.36-7.28(1 \mathrm{H}, \mathrm{m}, \mathrm{C}(5) \mathrm{H}), 6.90-6.82(1 \mathrm{H}, \mathrm{m}$, $\mathrm{C}(4) \mathrm{H}), 6.57-6.49(2 \mathrm{H}, \mathrm{m}, \mathrm{C}(2,6) \mathrm{H}), 2.02(3 \mathrm{H}, \mathrm{s}, \mathrm{Me}) \mathrm{ppm} .{ }^{19} \mathrm{~F}$ $N M R \delta F=-73.12--77.79\left(3 F, m, C F_{3}\right),-109.19--115.09(1 F$, $\mathrm{m}, F) \mathrm{ppm} .{ }^{13} \mathrm{C}$ NMR $\delta \mathrm{C}=163.44\left(\mathrm{~d},{ }^{1} J_{\mathrm{C}-F}=247.2 \mathrm{~Hz}, \mathrm{C}-3\right)$, $158.64\left(q,{ }^{2} J_{C-F}=34.3 \mathrm{~Hz}, C C_{3}\right), 149.33\left(\mathrm{~d},{ }^{3} J_{C-F}=9.1 \mathrm{~Hz}, \mathrm{C}-1\right)$, $130.70\left(\mathrm{~d},{ }^{3} J_{C-F}=9.2 \mathrm{~Hz}, \mathrm{C}-5\right), 119.56\left(\mathrm{q},{ }^{1} J_{C-F}=278.3 \mathrm{~Hz}, \mathrm{CF}_{3}\right)$, $114.45\left(\mathrm{~d},{ }^{4} J_{C-F}=2.9 \mathrm{~Hz}, \mathrm{C}-6\right), 112\left(\mathrm{~d},{ }^{2} J_{C-F}=21.3 \mathrm{~Hz}, \mathrm{C}-4\right)$, $106.38\left(\mathrm{~d},{ }^{2} J_{C-F}=24.1 \mathrm{~Hz}, \mathrm{C}-2\right), 14.36(\mathrm{~s}, \mathrm{Me}) \mathrm{ppm}$. HRMS (ESI +) for $\mathrm{C}_{9} \mathrm{H}_{8} \mathrm{~F}_{4} \mathrm{~N}[\mathrm{M}+\mathrm{H}]$ : calcd 206.0587, found 206.0572.

4-Fluoro-N-(1,1,1-trifluoropropan-2-ylidene)aniline 1g. The product was prepared according to the general procedure and starting from 1,1,1-trifluoroacetone (2 equiv., $1.87 \mathrm{~mL}, 20.8$ $\mathrm{mmol}$ ) and 4-fluoroaniline (1 equiv., $1 \mathrm{~mL}, 10.4 \mathrm{mmol}$ ) in presence of ( $4 \AA \mathrm{MS})$. The reaction mixture was stirred for $13 \mathrm{~h}$ at room temperature. 4-Fluoro- $N$-(1,1,1-trifluoropropan-2ylidene)aniline $1 \mathrm{~g}$ was provided as a brown oil (1.97 $\mathrm{g}, 93 \%)$. ${ }^{1} \mathrm{H} N M R \delta H=7.10-7.03(2 \mathrm{H}, \mathrm{m}, \mathrm{C}(3,5) \mathrm{H}), 6.79-6.73(2 \mathrm{H}, \mathrm{m}$,
$\mathrm{C}(2,6) \mathrm{H}), 2.03(3 \mathrm{H}, \mathrm{s}, \mathrm{Me}) \mathrm{ppm} .{ }^{19} \mathrm{~F} N \mathrm{NMR} \delta \mathrm{F}=-74.77--74.79$ $\left(3 \mathrm{~F}, \mathrm{~m}, \mathrm{CF}_{3}\right),-117.79--118.66(1 \mathrm{~F}, \mathrm{~m}, \mathrm{~F}) \mathrm{ppm} .{ }^{13} \mathrm{C} \mathrm{NMR} \delta \mathrm{C}=$ $160.39\left(\mathrm{~d},{ }^{1} J_{C-F}=244.1 \mathrm{~Hz}, \mathrm{C}-4\right), 158.01\left(\mathrm{qd},{ }^{2} J_{C-F}=33.9,6 J_{C-F}=\right.$ $\left.1.1 \mathrm{~Hz}, \mathrm{CCF}_{3}\right), 143.59\left(\mathrm{~d},{ }^{4} J_{C-F}=2.7 \mathrm{~Hz}, \mathrm{C}-1\right), 120.68\left(2 \mathrm{C}, \mathrm{d},{ }^{3} J_{C-F}\right.$ $=8.2 \mathrm{~Hz}, \mathrm{C}-2,6), 119.68\left(\mathrm{q},{ }^{1} J_{\mathrm{C}-F}=278.2 \mathrm{~Hz}, \mathrm{CF}_{3}\right), 115.98(2 \mathrm{C}, \mathrm{d}$, $\left.{ }^{2} J_{C-F}=22.8 \mathrm{~Hz}, \mathrm{C}-3,5\right), 14.38(\mathrm{~s}, \mathrm{Me}) \mathrm{ppm}$. LCMS (ESI -) for $\mathrm{C}_{9} \mathrm{H}_{6} \mathrm{~F}_{4} \mathrm{~N}[\mathrm{M}-\mathrm{H}] \mathrm{m} / \mathrm{z} 223(\mathrm{M}+\mathrm{H} 2 \mathrm{O}, 100 \%), 153$ (15).

3-(Trifluoromethoxy)- $\mathrm{N}$-(1,1,1-trifluoropropan-2-

ylidene)aniline $\mathbf{1 h}$. The product was prepared according to the general procedure and starting from 1,1,1-trifluoroacetone (2 equiv., $1.01 \mathrm{~mL}, 11.3 \mathrm{mmol}$ ) and 3-(trifluoromethoxy)aniline (1 equiv., $0.76 \mathrm{~mL}, 5.65 \mathrm{mmol}$ ) in presence of (4 $\AA \mathrm{MS})$. The reaction mixture was stirred for $48 \mathrm{~h}$ at room temperature. 3(Trifluoromethoxy)- $\mathrm{N}$-(1,1,1-trifluoropropan-2-ylidene)aniline 1h was provided as a brown oil $\left(1.25 \mathrm{~g}, 82 \%\right.$, estimated). ${ }^{1} \mathrm{H}$ NMR $\delta H=7.40\left(1 \mathrm{H}, \mathrm{t},{ }^{3} J_{H-H}=8.1 \mathrm{~Hz}, \mathrm{C}(5) \mathrm{H}\right), 7.04\left(1 \mathrm{H}, \mathrm{d},{ }^{3} J_{H-H}=\right.$ $8.3 \mathrm{~Hz}, \mathrm{C}(4) \mathrm{H}), 6.71\left(1 \mathrm{H}, \mathrm{d},{ }^{3} \mathrm{~J}_{\mathrm{H}-\mathrm{H}}=7.9 \mathrm{~Hz}, \mathrm{C}(6) \mathrm{H}\right), 6.68(1 \mathrm{H}, \mathrm{s}$, $\mathrm{C}(2) \mathrm{H}), 2.03(\mathrm{~s}, \mathrm{Me}) \mathrm{ppm} .{ }^{19} \mathrm{~F} \mathrm{NMR} \delta \mathrm{F}=-58.08\left(3 \mathrm{~F}, \mathrm{~s}, \mathrm{OCF}_{3}\right)$, $74.91\left(3 \mathrm{~F}, \mathrm{~s}, \mathrm{CF}_{3}\right) \mathrm{ppm} .{ }^{13} \mathrm{C}$ NMR $\delta \mathrm{C}=158.85\left(\mathrm{q},{ }^{2} \mathrm{~J}_{\mathrm{C}-\mathrm{F}}=34.3 \mathrm{~Hz}\right.$, $\left.C_{C C}\right), 150.05\left(q,{ }^{3} J_{C-F}=2.02 \mathrm{~Hz}, \mathrm{C}-3\right), 149.12(\mathrm{~s}, \mathrm{C}-1), 130.75(\mathrm{~s}$, C-5), $120.41\left(q,{ }^{1} J_{C-F}=258.2 \mathrm{~Hz}, \mathrm{OCF}_{3}\right), 119.49\left(\mathrm{q},{ }^{1} J_{C-F}=278.3\right.$ $\left.\mathrm{Hz}, \mathrm{CF}_{3},\right), 117.27$ (s, C-4), 116.96 (s, C-6), 111.58 (s, C-2), 14.52 (s, Me) ppm. HRMS (ESI +) for $\mathrm{C}_{10} \mathrm{H}_{8} \mathrm{~F}_{6} \mathrm{NO}[\mathrm{M}+\mathrm{H}]$ : calcd 272.0505 , found 272.0488 .

\section{4-(Trifluoromethoxy)- $\mathrm{N}$-(1,1,1-trifluoropropan-2-}

ylidene)aniline 1i. The product was prepared according to the general procedure and starting from 1,1,1-trifluoroacetone (2 equiv., $2.02 \mathrm{~mL}, 22.6 \mathrm{mmol}$ ) and 4-(trifluoromethoxy)aniline (1 equiv., $1.53 \mathrm{~mL}, 11.3 \mathrm{mmol}$ ) in presence of (4 $\AA \mathrm{MS})$. The reaction mixture was stirred for $48 \mathrm{~h}$ at room temperature. 4(Trifluoromethoxy)-N-(1,1,1-trifluoropropan-2-ylidene)aniline 1i was provided as a brown oil ( $2.85 \mathrm{~g}, 93 \%$, estimated), isomer ration 87:13. ${ }^{1} \mathrm{H} \mathrm{NMR} \delta \mathrm{H}=7.17\left(2 \mathrm{H}, \mathrm{d},{ }^{3} J_{\mathrm{H}-\mathrm{H}}=8.8 \mathrm{~Hz}, \mathrm{C}(2,6) \mathrm{H}\right)$, $6.95\left(2 \mathrm{H}, \mathrm{d},{ }^{3} \mathrm{~J}_{\mathrm{H}-\mathrm{H}}=8.8 \mathrm{~Hz}, \mathrm{C}\left(2^{\prime}, 6^{\prime}\right) \mathrm{H}\right), 6.73\left(2 \mathrm{H}, \mathrm{d},{ }^{3} J_{\mathrm{H}-\mathrm{H}}=8.8 \mathrm{~Hz}\right.$, $\mathrm{C}(3,5) \mathrm{H}), 6.61\left(2 \mathrm{H}, \mathrm{d}, 3^{3} \mathrm{H}_{\mathrm{H}}=8.8 \mathrm{~Hz}, \mathrm{C}\left(3^{\prime}, 5^{\prime}\right) \mathrm{H}\right), 1.96(3 \mathrm{H}, \mathrm{s}$, $\mathrm{Me}), 1.96\left(3 \mathrm{H}, \mathrm{s}, \mathrm{Me}^{\prime}\right)$ ppm. ${ }^{19} \mathrm{~F} N M R \delta \mathrm{F}=-58.40--58.67(3 \mathrm{~F}$, $\left.\mathrm{m}, \mathrm{OCF}_{3}\right),-74.89--75.12\left(3 \mathrm{~F}, \mathrm{~m}, \mathrm{CF}_{3}\right) \mathrm{ppm} .{ }^{13} \mathrm{C} \mathrm{NMR} \delta \mathrm{C}=$ $158.94\left(q,{ }^{2} J_{C-F}=34.3 \mathrm{~Hz}, C_{C F}\right), 146.84\left(q,{ }^{3} J_{C-F}=2.02 \mathrm{~Hz}, \mathrm{C}-4\right)$, 146.15 (s, C-1), 122.26 (2 C, s, C-2,6), 120.31 (2 C, s, C-3,5), $120.76\left(q,{ }^{1} J_{C-F}=256.8 \mathrm{~Hz}, \mathrm{OCF}_{3}\right), 119.79\left(\mathrm{q},{ }^{1} J_{C-F}=278.2 \mathrm{~Hz}\right.$, $\left.\mathrm{CF}_{3}\right), 14.46(\mathrm{~s}, \mathrm{Me}) \mathrm{ppm}$. HRMS (ESI + ) for $\mathrm{C}_{10} \mathrm{H}_{8} \mathrm{~F}_{6} \mathrm{NO}[\mathrm{M}+\mathrm{H}]$ : calcd 272.0505, found 272.0496 .

1-N,N-Dimethyl-4-N-(1,1,1-trifluoropropan-2-

ylidene)benzene-1,4-diamine $\mathbf{1} \mathbf{j}$. The product was prepared according to the general procedure and starting from 1,1,1trifluoroacetone (2 equiv., $1.32 \mathrm{~mL}, 14.8 \mathrm{mmol}$ ) and $\mathrm{N}, \mathrm{N}$ dimethyl-1,4-benzenediamine (1 equiv., $0.92 \mathrm{~mL}, 7.39 \mathrm{mmol}$ ) in presence of ( $4 \AA \mathrm{MS})$. The reaction mixture was stirred for $24 \mathrm{~h}$ at room temperature. 1- $N, N$-Dimethyl-4- $N-(1,1,1-$ trifluoropropan-2-ylidene)benzene-1,4-diamine $\mathbf{1 j}$ was provided as a brown oil (1.36 g, 80\%). ${ }^{1} \mathrm{H}$ NMR $\delta \mathrm{H}=6.83(2 \mathrm{H}$, m, AA', C(2,6)H), $6.74\left(2 \mathrm{H}, \mathrm{m}, \mathbf{B B}^{\prime}, \mathrm{C}(3,5) \mathrm{H}\right), 2.96(6 \mathrm{H}, \mathrm{s}$, $\left.\mathrm{NMe}_{2}\right), 2.11(3 \mathrm{H}, \mathrm{s}, \mathrm{Me}) \mathrm{ppm} .{ }^{19} \mathrm{~F} \mathrm{NMR} \delta \mathrm{F}=-74.19\left(3 \mathrm{~F}, \mathrm{~s}, \mathrm{CF}_{3}\right)$ ppm. ${ }^{13} \mathrm{C} N M R \delta C=154.86\left(q,{ }^{2} J_{C-F}=33.3 \mathrm{~Hz}, C C F_{3}\right), 148.94(\mathrm{~s}$, C-4), 136.65 (s, C-1), 121.94 (2 C, s, C-2,6), 120.15 (q, ${ }^{1} J_{C-F}=$ $278.0 \mathrm{~Hz}, \mathrm{CF}_{3}$ ), 112.86 (2 C, s, C-3,5), 40.84 (2 C, s, $\mathrm{NMe}_{2}$ ), 
14.67 (s, Me) ppm. HRMS (ESI +) for $\mathrm{C}_{11} \mathrm{H}_{14} \mathrm{~F}_{3} \mathrm{~N}_{2}[\mathrm{M}+\mathrm{H}]$ : calcd 231.1104, found 231.1125.

\section{3-Fluoro-2-methyl-N-(1,1,1-trifluoropropan-2-ylidene)aniline} 1k. The product was prepared according to the general procedure and starting from 1,1,1-trifluoroacetone ( 2 equiv., $2.87 \mathrm{~mL}, 32 \mathrm{mmol}$ ) and 3-fluoro-2-methylaniline (1 equiv., 1.82

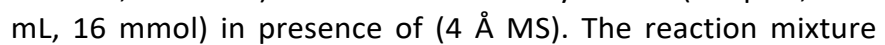
was stirred for $26 \mathrm{~h}$ at room temperature. 3-Fluoro-2-methyl$\mathrm{N}$-(1,1,1-trifluoropropan-2-ylidene)aniline $\mathbf{1} \mathbf{k}$ was provided as a brown oil ( $2.01 \mathrm{~g}, 57 \%$, estimated). ${ }^{1} \mathrm{H}$ NMR $\delta \mathrm{H}=7.17(1 \mathrm{H}, \mathrm{dd}$, $\left.{ }^{3} J_{H-F}=14.3,3 J_{H-H}=7.8 \mathrm{~Hz}, \mathrm{C}(4) \mathrm{H}\right), 6.97\left(1 \mathrm{H}, \mathrm{dd},{ }^{4} J_{H-F}=14.8,{ }^{3} J_{H-H}\right.$ $=7.8 \mathrm{~Hz}, \mathrm{C}(5) \mathrm{H}), 6.43\left(1 \mathrm{H}, \mathrm{d}, 3_{\mathrm{H}-\mathrm{H}}=7.9 \mathrm{~Hz}, \mathrm{C}(6) \mathrm{H}\right), 2.01(3 \mathrm{H}, \mathrm{s}$, $\mathrm{C}(2) \mathrm{HMe}), 1.99(3 \mathrm{H}, \mathrm{s}, \mathrm{Me}) \mathrm{ppm} .{ }^{19} \mathrm{~F} \mathrm{NMR} \delta \mathrm{F}=-74.56(3 \mathrm{~F}, \mathrm{~s}$, $\left.\mathrm{CF}_{3}\right),-115.94(1 \mathrm{~F}, \mathrm{~s}, \mathrm{~F})$ ppm. HRMS $(\mathrm{ESI}+)$ for $\mathrm{C}_{10} \mathrm{H}_{10} \mathrm{~F}_{4} \mathrm{~N}[\mathrm{M}+$ $\mathrm{H}]$ : calcd 220.0744, found 220.0746 .

3-Chloro-2-methyl-N-(1,1,1-trifluoropropan-2-ylidene)aniline 11. The product was prepared according to the general procedure and starting from 1,1,1-trifluoroacetone (2 equiv., $2.53 \mathrm{~mL}, 28.2 \mathrm{mmol}$ ) and 3-chloro-2-methylaniline (1 equiv.

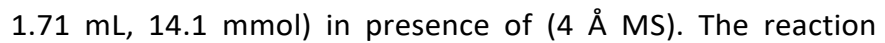
mixture was stirred for $26 \mathrm{~h}$ at room temperature. 3-Chloro-2methyl- $N$-(1,1,1-trifluoropropan-2-ylidene)aniline $\mathbf{1 l}$ was provided as a brown oil (1.67 g, 50\%, estimated). ${ }^{1} \mathrm{H}$ NMR $\delta \mathrm{H}=$ $7.22\left(1 \mathrm{H}, \mathrm{dd},{ }^{3} J_{H-H}=8.0,4 J_{H-H}=1.0 \mathrm{~Hz}, \mathrm{C}(4) \mathrm{H}\right), 7.14\left(1 \mathrm{H}, \mathrm{t},{ }^{3} \mathrm{~J}_{\mathrm{H}-\mathrm{H}}\right.$ $=7.9 \mathrm{~Hz}, \mathrm{C}(5) \mathrm{H}), 6.53\left(1 \mathrm{H}, \mathrm{dd},{ }^{3} J_{H-H}=7.8,{ }^{4} J_{H-H}=0.8 \mathrm{~Hz}, \mathrm{C}(6) \mathrm{H}\right)$, $2.14(3 \mathrm{H}, \mathrm{s}, \mathrm{C}(2) \mathrm{HMe}), 1.98(3 \mathrm{H}, \mathrm{s}, \mathrm{Me}) \mathrm{ppm} .{ }^{19} \mathrm{~F} N M R \delta \mathrm{F}=-$ $74.51\left(3 \mathrm{~F}, \mathrm{~s}, \mathrm{CF}_{3}\right)$ ppm. HRMS (ESI +) for $\mathrm{C}_{10} \mathrm{H}_{10} \mathrm{~F}_{3} \mathrm{NCl}[\mathrm{M}+\mathrm{H}]$ : calcd 236.0448, found 236.0462 .

$\mathbf{N}$-(1,1-Difluoropropan-2-ylidene)aniline 1aa. The product was prepared according to the general procedure and starting from 1,1-difluoroacetone (1.07 equiv., $5 \mathrm{~mL}, 58.2 \mathrm{mmol}$ ) and aniline (1 equiv., $5 \mathrm{~mL}, 54.6 \mathrm{mmol}$ ) in presence of (4 $\AA \mathrm{MS})$. The reaction mixture was stirred for $3 \mathrm{~h}$ at room temperature. $\mathrm{N}$ (1,1-Difluoropropan-2-ylidene)aniline 1aa was provided as a brown oil $(8.72 \mathrm{~g}, 96 \%) .{ }^{1} \mathrm{H} \mathrm{NMR} \delta \mathrm{H}=7.37\left(2 \mathrm{H}, \mathrm{t},{ }^{3} J_{\mathrm{H}-\mathrm{H}}=7.8\right.$ $\mathrm{Hz}, \mathrm{C}(3,5) \mathrm{H}), 7.16\left(1 \mathrm{H}, \mathrm{t}, 3 \mathrm{~J}_{\mathrm{H}-\mathrm{H}}=7.2 \mathrm{~Hz}, \mathrm{C}(4) \mathrm{H}\right), 6.78\left(2 \mathrm{H}, \mathrm{d}, 3^{3} \mathrm{H}_{-}\right.$ $H=7.4 \mathrm{~Hz}, \mathrm{C}(2,6) \mathrm{H}), 6.07\left(1 \mathrm{H}, \mathrm{t},{ }^{2} J_{H-F}=55.6 \mathrm{~Hz}, \mathrm{CHF}_{2}\right), 1.95(3$ $\mathrm{H}, \mathrm{s}, \mathrm{Me}) \mathrm{ppm} .{ }^{19} \mathrm{~F} N M R \delta \mathrm{F}=-121.27\left(2 \mathrm{~F}, \mathrm{~d},{ }^{2} \mathrm{~J}_{\mathrm{F}-\mathrm{H}}=55.5 \mathrm{~Hz}\right.$, $\left.\mathrm{CHF}_{2}\right) \mathrm{ppm} .{ }^{13} \mathrm{C} N M R \delta \mathrm{C}=163.54\left(\mathrm{t},{ }^{2} \mathrm{~J}_{\mathrm{C}-\mathrm{F}}=28.8 \mathrm{~Hz}, \mathrm{CCHF}_{2}\right)$, 148.47 (s, C-1), 129.12 (2 C, s, C-3,5), 124.86 (s, C-4), 119.01 (2 C, s, C-2,6), $114.96\left(\mathrm{t},{ }^{1} J_{\mathrm{C}-\mathrm{F}}=243.0 \mathrm{~Hz}, \mathrm{CHF}_{2}\right), 12.65(\mathrm{~s}, \mathrm{Me})$ ppm. HRMS $(E S I+)$ for $\mathrm{C}_{9} \mathrm{H}_{10} \mathrm{~F}_{2} \mathrm{~N}[\mathrm{M}+\mathrm{H}]$ : calcd 170.0774, found 170.0776 .

$\mathbf{N}$-(1,1-Difluoropropan-2-ylidene)-2-methoxyaniline $\mathbf{1 b b}$. The product was prepared according to the general procedure and starting from 1,1-difluoroacetone (2 equiv., $1.32 \mathrm{~mL}, 16.2$ $\mathrm{mmol}$ ) and 2-methoxyaniline (1 equiv., $0.92 \mathrm{~mL}, 8.12 \mathrm{mmol}$ ) in presence of ( $4 \AA \mathrm{MS})$. The reaction mixture was stirred for $5 \mathrm{~h}$ at room temperature. $N$-(1,1-Difluoropropan-2-ylidene)-2methoxyaniline $\mathbf{1 b b}$ was provided as a brown oil (1.59 g, 98\%). ${ }^{1} \mathrm{H} N M R \delta H=7.14\left(1 \mathrm{H}, \mathrm{td},{ }^{3} J_{H-H}=7.8,{ }^{4} J_{H-H}=1.6 \mathrm{~Hz}, \mathrm{C}(4) \mathrm{H}\right)$, $6.99-6.92(2 \mathrm{H}, \mathrm{m}, \mathrm{C}(5,6) \mathrm{H}), 6.74\left(1 \mathrm{H}, \mathrm{dd},{ }^{3} \mathrm{~J}_{\mathrm{H}-\mathrm{H}}=7.6,{ }^{4} \mathrm{~J}_{\mathrm{H}-\mathrm{H}}=\right.$ $1.6 \mathrm{~Hz}, \mathrm{C}(3) \mathrm{H}), 6.12\left(1 \mathrm{H}, \mathrm{t},{ }^{2} J_{\mathrm{H}-\mathrm{F}}=55.6 \mathrm{~Hz}, \mathrm{CHF}_{2}\right), 3.80(3 \mathrm{H}, \mathrm{s}$, OMe), $1.89(3 \mathrm{H}, \mathrm{s}, \mathrm{Me}) \mathrm{ppm} .{ }^{19} \mathrm{~F} N M R \delta \mathrm{F}=-120.86\left(2 \mathrm{~F}, \mathrm{~d},{ }^{2} J_{F-H}\right.$ $\left.=55.5 \mathrm{~Hz}, \mathrm{CHF}_{2}\right) \mathrm{ppm} .{ }^{13} \mathrm{C} \mathrm{NMR} \delta \mathrm{C}=164.88\left(\mathrm{t},{ }^{2} \mathrm{~J}_{\mathrm{C}-\mathrm{F}}=28.6 \mathrm{~Hz}\right.$, $\mathrm{CCHF}_{2}$ ), 148.64 (s, C-2), 137.30 (s, C-1), 125.75 (s, C-4), 120.87 (s, C-5), $120.17(\mathrm{~s}, \mathrm{C}-3), 114.83\left(\mathrm{t},{ }^{1} \mathrm{~J}_{\mathrm{C}-\mathrm{F}}=242.8 \mathrm{~Hz}, \mathrm{CHF}_{2}\right.$ ),
111.65 (s, C-6), 55.56 (s, OMe), 13.11 (s, Me) ppm. HRMS (ESI + ) for $\mathrm{C}_{10} \mathrm{H}_{12} \mathrm{~F}_{2} \mathrm{NO}[\mathrm{M}+\mathrm{H}]$ : calcd 200.0881, found 200.0875 .

$\mathbf{N}$-(1,1-Difluoropropan-2-ylidene)-3-methoxyaniline 1cc. The product was prepared according to the general procedure and starting from 1,1-difluoroacetone (2 equiv., $1.32 \mathrm{~mL}, 16.2$ $\mathrm{mmol}$ ) and 3-methoxyaniline (1 equiv., $0.91 \mathrm{~mL}, 8.12 \mathrm{mmol}$ ) in presence of ( $4 \AA \mathrm{MS})$. The reaction mixture was stirred for $4 \mathrm{~h}$ at room temperature. $\mathrm{N}$-(1,1-Difluoropropan-2-ylidene)-3methoxyaniline 1cc was provided as a brown oil (1.58 $\mathrm{g}, 98 \%)$. ${ }^{1} \mathrm{H}$ NMR $(\delta \mathrm{H}=7.30-7.26(1 \mathrm{H}, \mathrm{m}, \mathrm{C}(5) \mathrm{H}), 6.75-6.73(1 \mathrm{H}, \mathrm{m}$, $\mathrm{C}(4) \mathrm{H}), 6.39-6.38(2 \mathrm{H}, \mathrm{m}, \mathrm{C}(2,6) \mathrm{H}), 6.10\left(1 \mathrm{H}, \mathrm{t},{ }^{2} \mathrm{~J}_{\mathrm{H}-\mathrm{F}}=55.6\right.$ $\left.\mathrm{Hz}, \mathrm{CHF}_{2}\right), 3.83-3.80(3 \mathrm{H}, \mathrm{m}, \mathrm{OMe}), 1.99-1.97(3 \mathrm{H}, \mathrm{m}, \mathrm{Me})$ ppm. ${ }^{19} \mathrm{~F} N M R \delta F=-121.26--121.44\left(2 \mathrm{~F}, \mathrm{~m},{ }^{2} J_{F-H}=55.6 \mathrm{~Hz}\right.$, $\mathrm{CHF}_{2}$ ppm. ${ }^{13} \mathrm{C}$ NMR $\delta \mathrm{C}=163.41\left(\mathrm{t},{ }^{2} J_{\mathrm{C}-\mathrm{F}}=28.7 \mathrm{~Hz}, \mathrm{CCHF}_{2}\right)$, 160.41 (s, C-3), 149.81 (s, C-1), 129.95 (s, C-5), 114.89 (t, $1^{1} \mathrm{C}_{\mathrm{C}-\mathrm{F}}=$ $242.9 \mathrm{~Hz}, \mathrm{CHF}_{2}$ ), 110.92 (s, C-6), 110.25 (s, C-4), 104.65 (s, C-2), 55.01 (s, OMe), 12.35 (s, Me) ppm. HRMS (ESI +) for $\mathrm{C}_{10} \mathrm{H}_{12} \mathrm{~F}_{2} \mathrm{NO}[\mathrm{M}+\mathrm{H}]$ : calcd 200.0881, found 200.0884.

$\mathbf{N}$-(1,1-Difluoropropan-2-ylidene)-4-methoxyaniline 1dd. The product was prepared according to the general procedure and starting from 1,1-difluoroacetone (2 equiv., $1.44 \mathrm{~mL}, 17.8$ $\mathrm{mmol}$ ) and 4-methoxyaniline (1 equiv., $8.91 \mathrm{mmol}$ ) in presence of ( $4 \AA \mathrm{MS})$. The reaction mixture was stirred for $5 \mathrm{~h}$ at room temperature. $\quad \mathrm{N}$-(1,1-Difluoropropan-2-ylidene)-4methoxyaniline 1dd was provided as a brown oil $(1.74 \mathrm{~g}, 98 \%$, estimated). ${ }^{1} \mathrm{H} N M R \delta H=6.87\left(2 \mathrm{H}, \mathrm{m}, \mathbf{A A}^{\prime}, \mathrm{C}(2,6) \mathrm{H}\right), 6.73(2 \mathrm{H}$, $\left.\mathrm{m}, \mathrm{BB}^{\prime}, \mathrm{C}(3,5) \mathrm{H}\right), 6.02\left(1 \mathrm{H}, \mathrm{t},{ }^{2} \mathrm{~J}_{\mathrm{H}-\mathrm{F}}=55.7 \mathrm{~Hz}, \mathrm{CHF}_{2}\right), 3.77-3.69$ ( $3 \mathrm{H}, \mathrm{m}, \mathrm{OMe}), 1.94-1.93(3 \mathrm{H}, \mathrm{m}, \mathrm{Me}) \mathrm{ppm} .{ }^{19} \mathrm{~F} \mathrm{NMR} \delta \mathrm{F}=-$ $121.11\left(2 \mathrm{~F}, \mathrm{dd}, 2 J_{F-H}=55.8,4 J_{F-H}=4.0 \mathrm{~Hz}, \mathrm{CHF}_{2}\right) \mathrm{ppm} .{ }^{13} \mathrm{C} \mathrm{NMR}$ $\delta \mathrm{C}=162.77\left(\mathrm{t},{ }^{2} J_{C-F}=29.4 \mathrm{~Hz}, C_{C H F}\right), 157.11(\mathrm{~s}, \mathrm{C}-4), 141.22(\mathrm{~s}$, $\mathrm{C}-1), 120.80(2 \mathrm{C}, \mathrm{s}, \mathrm{C}-3,5), 115.23\left(\mathrm{t},{ }^{1} \mathrm{~J}_{\mathrm{C}-\mathrm{F}}=242.7 \mathrm{~Hz}, \mathrm{CHF}_{2}\right)$, 114.21 (2 C, s, C-2,6), 55.11 (s, OMe), 12.26 (s, Me) ppm. HRMS $(\mathrm{ESI}+)$ for $\mathrm{C}_{10} \mathrm{H}_{12} \mathrm{~F}_{2} \mathrm{NO}[\mathrm{M}+\mathrm{H}]$ : calcd 200.0881, found 200.0893.

$\boldsymbol{N}$-(1,1-Difluoropropan-2-ylidene)-2-fluoroaniline 1ee. The product was prepared according to the general procedure and starting from 1,1-difluoroacetone (2 equiv., $2.92 \mathrm{~mL}, 36 \mathrm{mmol}$ ) and 2-fluoroaniline (1 equiv., $1.74 \mathrm{~mL}, 18 \mathrm{mmol}$ ) in presence of (4 $\AA \mathrm{MS})$. The reaction mixture was stirred for $4 \mathrm{~h}$ at room temperature. $\mathrm{N}$-(1,1-Difluoropropan-2-ylidene)-2-fluoroaniline 1ee was provided as a brown oil $\left(2.62 \mathrm{~g}, 78 \%\right.$, estimated). ${ }^{1} \mathrm{H}$ NMR $\delta H=7.19-7.08(3 \mathrm{H}, \mathrm{m}, \mathrm{C}(4,5,6) \mathrm{H}), 6.90-6.82(1 \mathrm{H}, \mathrm{m}$, $\mathrm{C}(3) \mathrm{H}), 6.10\left(1 \mathrm{H}, \mathrm{t},{ }^{2} \mathrm{~J}_{\mathrm{H}-\mathrm{F}}=55.5 \mathrm{~Hz}, \mathrm{CHF}_{2}\right), 1.99-1.91(3 \mathrm{H}, \mathrm{m}$, Me) ppm. ${ }^{19} \mathrm{~F} N M R \delta F=-121.24\left(2 \mathrm{~F}, \mathrm{~d},{ }^{2} \mathrm{~J}_{\mathrm{F}-\mathrm{H}}=55.5 \mathrm{~Hz}, \mathrm{CHF}_{2}\right)$, $126.63(1 \mathrm{~F}, \mathrm{~s}, \mathrm{~F}) \mathrm{ppm} .{ }^{13} \mathrm{C} \mathrm{NMR} \delta \mathrm{C}=166.37\left(\mathrm{t},{ }^{2} J_{C-F}=28.9 \mathrm{~Hz}\right.$, $\left.C_{C H F}\right), 151.51\left(d,{ }^{1} J_{C-F}=245.8 \mathrm{~Hz}, \mathrm{C}-2\right), 135.82\left(\mathrm{~d},{ }^{2} J_{C-F}=13.0\right.$ $\mathrm{Hz}, \mathrm{C}-1), 126.07\left(\mathrm{~d},{ }^{3} J_{C-F}=7.3 \mathrm{~Hz}, \mathrm{C}-4\right), 124.48\left(\mathrm{~d},{ }^{4} J_{C-F}=3.8 \mathrm{~Hz}\right.$, C-5), $121.72(\mathrm{~s}, \mathrm{C}-6), 116.11$ (d, $\left.{ }^{2} J_{\mathrm{C}-F}=19.8 \mathrm{~Hz}, \mathrm{C}-3\right), 114.49$ (t, $\left.{ }^{1} J_{C-F}=243.0 \mathrm{~Hz}, \mathrm{CHF}_{2}\right), 13.14(\mathrm{~s}, \mathrm{Me})$ ppm. HRMS (ESI +) for $\mathrm{C}_{9} \mathrm{H}_{9} \mathrm{~F}_{3} \mathrm{~N}[\mathrm{M}+\mathrm{H}]$ : calcd 188.0682, found 188.0687 .

$\mathbf{N}$-(1,1-Difluoropropan-2-ylidene)-3-fluoroaniline 1ff. The product was prepared according to the general procedure and starting from 1,1-difluoroacetone (2 equiv., $1.46 \mathrm{~mL}, 18 \mathrm{mmol}$ ) and 3-fluoroaniline (1 equiv., $0.87 \mathrm{~mL}, 9 \mathrm{mmol}$ ) in presence of (4 $\AA \mathrm{MS})$. The reaction mixture was stirred for $5 \mathrm{~h}$ at room temperature. $\mathrm{N}$-(1,1-difluoropropan-2-ylidene)-3-fluoroaniline 1 ff was provided as a brown oil (1.53 g, 91\%). ${ }^{1} \mathrm{H} \mathrm{NMR} \delta \mathrm{H}=$ 
$7.33-7.28(1 \mathrm{H}, \mathrm{m}, \mathrm{C}(5) \mathrm{H}), 6.84\left(1 \mathrm{H}, \mathrm{td},{ }^{3} J_{H-H}=8.5,{ }^{4} J_{H-H}=2.5\right.$ $\mathrm{Hz}, \mathrm{C}(4) \mathrm{H}), 6.55-6.49(2 \mathrm{H}, \mathrm{m}, \mathrm{C}(2,6) \mathrm{H}), 6.03\left(1 \mathrm{H}, \mathrm{t},{ }^{2} J_{H-F}=\right.$ $\left.55.5 \mathrm{~Hz}, \mathrm{CHF}_{2}\right), 1.94(3 \mathrm{H}, \mathrm{s}, \mathrm{Me}) \mathrm{ppm} .{ }^{19} \mathrm{~F} N M R \delta \mathrm{F}=-111.96-$ $112.07(1 \mathrm{~F}, \mathrm{~m}, \mathrm{~F}),-121.51\left(2 \mathrm{~F}, \mathrm{~d},{ }^{2} J_{F-H}=55.4 \mathrm{~Hz}, \mathrm{CHF}_{2}\right) \mathrm{ppm}$. ${ }^{13} \mathrm{C} N M R \delta C=164.38\left(\mathrm{t},{ }^{2} J_{C-F}=28.9 \mathrm{~Hz}, C C \mathrm{CHF}_{2}\right), 163.35\left(\mathrm{~d},{ }^{1} J_{C-F}=\right.$ $247.45 \mathrm{~Hz}, \mathrm{C}-3), 150.28\left(\mathrm{~d},{ }^{3} J_{C-F}=9.2 \mathrm{~Hz}, \mathrm{C}-1\right), 130.63\left(\mathrm{~d},{ }^{3} J_{C-F}=\right.$ $9.2 \mathrm{~Hz}, \mathrm{C}-5), 114.57\left(\mathrm{t},{ }^{1} J_{C-F}=242.4 \mathrm{~Hz}, \mathrm{CHF}_{2}\right), 114.48\left(\mathrm{~d},{ }^{4} J_{C-F}=\right.$ $2.9 \mathrm{~Hz}, \mathrm{C}-6), 111.38\left(\mathrm{~d}, 2 J_{C-F}=21.3 \mathrm{~Hz}, \mathrm{C}-4\right), 106.36\left(\mathrm{~d},{ }^{2} J_{C-F}=\right.$ $23.7 \mathrm{~Hz}, \mathrm{C}-2), 12.65$ (s, Me) ppm. HRMS (ESI +) for $\mathrm{C}_{9} \mathrm{H}_{9} \mathrm{~F}_{3} \mathrm{~N}[\mathrm{M}$ $+\mathrm{H}]$ : calcd 188.0682, found 188.0672 .

$\mathbf{N}$-(1,1-Difluoropropan-2-ylidene)-4-fluoroaniline 1gg. The product was prepared according to the general procedure and starting from 1,1-difluoroacetone (2 equiv., $1.35 \mathrm{~mL}, 16.7$ $\mathrm{mmol}$ ) and 4-fluoroaniline (1 equiv., $0.8 \mathrm{~mL}, 8.33 \mathrm{mmol}$ ) in presence of ( $4 \AA \mathrm{MS})$. The reaction mixture was stirred for $3 \mathrm{~h}$ at room temperature. $\mathrm{N}$-(1,1-Difluoropropan-2-ylidene)-4fluoroaniline $1 \mathrm{gg}$ was provided as a brown oil (1.29 g, 83\%). ${ }^{1} \mathrm{H}$ $\mathrm{NMR} \delta \mathrm{H}=7.04\left(2 \mathrm{H}, \mathrm{t},{ }^{3} J_{\mathrm{H}-\mathrm{H}}=8.7 \mathrm{~Hz}, \mathrm{C}(2,6) \mathrm{H}\right), 6.75-6.72(2 \mathrm{H}$, $\mathrm{m}, \mathrm{C}(3,5) \mathrm{H}), 6.02\left(1 \mathrm{H}, \mathrm{t},{ }^{2} \mathrm{~J}_{\mathrm{H}-\mathrm{F}}=55.6 \mathrm{~Hz}, \mathrm{CHF}_{2}\right), 1.94(3 \mathrm{H}, \mathrm{s}, \mathrm{Me})$ ppm. ${ }^{19} \mathrm{~F} N M R \delta F=-118.69--119.06(1 \mathrm{~F}, \mathrm{~m}, \mathrm{~F}),-121.38(2 \mathrm{~F}$, $\left.\mathrm{dd},{ }^{2} J_{F-H}=55.6,4 J_{F-H}=2.6 \mathrm{~Hz}, \mathrm{CHF}_{2}\right) \mathrm{ppm} .{ }^{13} \mathrm{C} \mathrm{NMR} \delta \mathrm{C}=164.10$ $\left(\mathrm{t},{ }^{2} J_{\mathrm{C}-F}=28.2 \mathrm{~Hz}, C \mathrm{CHF}_{2}\right), 160.27\left(\mathrm{~d},{ }^{1} J_{\mathrm{C}-F}=243.3 \mathrm{~Hz}, \mathrm{C}-4\right)$, $144.44\left(\mathrm{~d},{ }^{4} J_{C-F}=1.2, \mathrm{C}-1\right), 120.60\left(2 \mathrm{C}, \mathrm{d},{ }^{3} J_{C-F}=8.1 \mathrm{~Hz}, \mathrm{C}-2,6\right)$, $115.86\left(2 \mathrm{C}, \mathrm{d},{ }^{2} J_{C-F}=22.6 \mathrm{~Hz}, \mathrm{C}-3,5\right), 114.82\left(\mathrm{t},{ }^{1} J_{C-F}=243.0 \mathrm{~Hz}\right.$, $\mathrm{CHF}_{2}$ ), 12.57 (s, Me) ppm. HRMS (ESI -) for $\mathrm{C}_{9} \mathrm{H}_{7} \mathrm{~F}_{3} \mathrm{~N}[\mathrm{M}-\mathrm{H}]$ : calcd 186.0525, found 186.0544 .

$\mathbf{N}$-(1,1-Difluoropropan-2-ylidene)-2-(trifluoromethoxy)aniline 1hh. The product was prepared according to the general procedure and starting from 1,1-difluoroacetone (2 equiv., $1.83 \mathrm{~mL}, 22.6 \mathrm{mmol}$ ) and 2-(trifluoromethoxy)aniline (1 equiv., $1.54 \mathrm{~mL}, 11.3 \mathrm{mmol}$ ) in presence of (4 $\AA \mathrm{MS})$. The reaction mixture was stirred for $5 \mathrm{~h}$ at room temperature. $\mathrm{N}-(1,1-$ Difluoropropan-2-ylidene)-2-(trifluoromethoxy)aniline $1 \mathrm{hh}$ was provided as a brown oil ( $2.13 \mathrm{~g}, 75 \%) .{ }^{1} \mathrm{H} \mathrm{NMR} \delta \mathrm{H}=7.27-7.18$ $(2 \mathrm{H}, \mathrm{m}, \mathrm{C}(4,6) \mathrm{H}), 7.12-7.06(1 \mathrm{H}, \mathrm{m}, \mathrm{C}(5) \mathrm{H}), 6.78-6.73(1 \mathrm{H}$, $\mathrm{m}, \mathrm{C}(3) \mathrm{H}), 6.03\left(1 \mathrm{H}, \mathrm{t}^{2} \mathrm{~J}_{\mathrm{H}-\mathrm{F}}=55.5 \mathrm{~Hz}, \mathrm{CHF}_{2}\right), 1.85(3 \mathrm{H}, \mathrm{s}, \mathrm{Me})$ ppm. ${ }^{19} \mathrm{~F} N M R \delta F=-58.46\left(3 \mathrm{~F}, \mathrm{~s}, \mathrm{OCF}_{3}\right),-121.95\left(2 \mathrm{~F}, \mathrm{~d},{ }^{2} J_{F-H}=\right.$ $\left.55.4 \mathrm{~Hz}, \mathrm{CHF}_{2}\right) \mathrm{ppm} .{ }^{13} \mathrm{C} \mathrm{NMR} \delta \mathrm{C}=166.44\left(\mathrm{t},{ }^{2} J_{\mathrm{C}-\mathrm{F}}=29.1 \mathrm{~Hz}\right.$, $\left.\mathrm{CCHF}_{2}\right), 141.52$ (s, C-2), 138.31 (s, C-1), 127.75 (s, C-4), 125.82 (s, C-5), 122.38 (s, C-6), $122.14\left(\mathrm{q},{ }^{1} J_{C-F}=234.3 \mathrm{~Hz}, \mathrm{OCF}_{3}\right.$ ), $120.96(\mathrm{~s}, \mathrm{C}-3), 114.62\left(\mathrm{t},{ }^{1} J_{\mathrm{C}-\mathrm{F}}=242.9 \mathrm{~Hz}, \mathrm{CHF}_{2}\right), 12.96(\mathrm{~s}, \mathrm{Me})$ ppm. HRMS (ESI positive) for $\mathrm{C}_{10} \mathrm{H}_{9} \mathrm{~F}_{5} \mathrm{NO}[\mathrm{M}+\mathrm{H}]$ : calcd 254.0599, found 254.0622.

$\mathbf{N}$-(1,1-Difluoropropan-2-ylidene)-3-(trifluoromethoxy)aniline 1ii. The product was prepared according to the general procedure and starting from 1,1-difluoroacetone (2 equiv., $0.92 \mathrm{~mL}, 11.3 \mathrm{mmol}$ ) and 3-(trifluoromethoxy)aniline (1 equiv., $0.76 \mathrm{~mL}, 5.65 \mathrm{mmol}$ ) in presence of (4 $\AA \mathrm{MS})$. The reaction mixture was stirred for $7 \mathrm{~h}$ at room temperature. $\mathrm{N}$-(1,1Difluoropropan-2-ylidene)-3-(trifluoromethoxy)aniline 1ii was provided as a brown oil (1.32 g, 93\%). ${ }^{1} \mathrm{H} \mathrm{NMR} \delta \mathrm{H}=7.37(1 \mathrm{H}$, $\left.\mathrm{t},{ }^{3} \mathrm{~J}_{\mathrm{H}-\mathrm{H}}=8.1 \mathrm{~Hz}, \mathrm{C}(5) \mathrm{H}\right), 7.04-6.99(1 \mathrm{H}, \mathrm{m}, \mathrm{C}(4) \mathrm{H}), 6.69(1 \mathrm{H}, \mathrm{d}$, $\left.3^{3} J_{H-H}=7.9 \mathrm{~Hz}, \mathrm{C}(6) \mathrm{H}\right), 6.66(1 \mathrm{H}, \mathrm{s}, \mathrm{C}(2) \mathrm{H}), 6.03\left(1 \mathrm{H}, \mathrm{t}, 2 J_{\mathrm{H}-\mathrm{F}}=\right.$ $\left.55.5 \mathrm{~Hz}, \mathrm{CHF}_{2}\right), 1.94(3 \mathrm{H}, \mathrm{s}, \mathrm{Me}) \mathrm{ppm} .{ }^{19} \mathrm{~F} \mathrm{NMR} \delta \mathrm{F}=-58.10(3 \mathrm{~F}$, $\left.\mathrm{s}, \mathrm{OCF}_{3}\right),-121.65\left(2 \mathrm{~F}, \mathrm{~d},{ }^{2} \mathrm{~J}_{F-H}=55.6 \mathrm{~Hz}, \mathrm{CHF}_{2}\right) .{ }^{13} \mathrm{C} \mathrm{NMR} \delta \mathrm{C}=$ $164.80\left(\mathrm{t},{ }^{2} J_{C-F}=29.0 \mathrm{~Hz}, \mathrm{CCHF}_{2}\right), 150.07-150.02(2 \mathrm{C}, \mathrm{m}, \mathrm{C}-$ 1,3), $130.57(\mathrm{~s}, \mathrm{C}-5), 121.72\left(\mathrm{q},{ }^{1} J_{C-F}=258.6 \mathrm{~Hz}, \mathrm{OCF}_{3}\right), 117.37$ (s, C-4), $117.00(\mathrm{~s}, \mathrm{C}-6), 114.68\left(\mathrm{t},{ }^{1} \mathrm{~J}_{\mathrm{C}-\mathrm{F}}=243.2 \mathrm{~Hz}, \mathrm{CHF}_{2}\right)$, $111.92(\mathrm{~s}, \mathrm{C}-2), 12.74(\mathrm{~s}, \mathrm{Me}) \mathrm{ppm}$. HRMS (ESI +) for $\mathrm{C}_{10} \mathrm{H}_{9} \mathrm{~F}_{5} \mathrm{NO}$ $[\mathrm{M}+\mathrm{H}]$ : calcd 254.0599, found 254.0583.

$\mathbf{N}$-(1,1-Difluoropropan-2-ylidene)-4-(trifluoromethoxy)aniline $\mathbf{1 j j}$. The product was prepared according to the general procedure and starting from 1,1-difluoroacetone (2 equiv., $1.83 \mathrm{~mL}, 22.6 \mathrm{mmol}$ ) and 4-(trifluoromethoxy)aniline (1 equiv., $1.53 \mathrm{~mL}, 11.3 \mathrm{mmol}$ ) in presence of (4 $\AA \mathrm{MS})$. The reaction mixture was stirred for $3 \mathrm{~h}$ at room temperature. $\mathrm{N}$-(1,1Difluoropropan-2-ylidene)-4-(trifluoromethoxy)aniline $\mathbf{1} \mathbf{j j}$ was provided as a brown oil (2.59 g, 91\%). ${ }^{1} \mathrm{H} \mathrm{NMR} \delta \mathrm{H}=7.22(2 \mathrm{H}$, $\left.\mathrm{d}, 3^{3} \mathrm{H}_{-H}=8.6 \mathrm{~Hz}, \mathrm{C}(2,6) \mathrm{H}\right), 6.78\left(2 \mathrm{H}, \mathrm{d}, 3 \mathrm{~J}_{\mathrm{H}-\mathrm{H}}=8.8 \mathrm{~Hz}, \mathrm{C}(3,5) \mathrm{H}\right)$, $6.03\left(1 \mathrm{H}, \mathrm{t},{ }^{2} J_{\mathrm{H}-\mathrm{F}}=55.6 \mathrm{~Hz}, \mathrm{CHF}_{2}\right), 1.94(3 \mathrm{H}, \mathrm{s}, \mathrm{Me}) \mathrm{ppm} .{ }^{19} \mathrm{~F}$ $\mathrm{NMR} \delta \mathrm{F}=-58.44\left(3 \mathrm{~F}, \mathrm{~s}, \mathrm{OCF}_{3}\right),-121.67\left(2 \mathrm{~F}, \mathrm{dd},{ }^{2} J_{F-H}=55.6,{ }^{4} J_{F-}\right.$ $\left.H_{H}=2 \mathrm{~Hz}, \mathrm{CHF}_{2}\right) \mathrm{ppm} .{ }^{13} \mathrm{C} \mathrm{NMR} \delta \mathrm{C}=164.54\left(\mathrm{t},{ }^{2} J_{\mathrm{C}-F}=28.9 \mathrm{~Hz}\right.$, $\left.\mathrm{CCHF}_{2}\right), 147.21(\mathrm{~s}, \mathrm{C}-1), 146.39\left(\mathrm{q},{ }^{3} \mathrm{~J}_{\mathrm{C}-F}=2 \mathrm{~Hz}, \mathrm{C}-4\right), 122.10(2 \mathrm{C}$, $\mathrm{s}, \mathrm{C}-2,6), 120.76\left(\mathrm{q},{ }^{1} \mathrm{~J}_{\mathrm{C}-F}=256.7 \mathrm{~Hz}, \mathrm{OCF}_{3}\right), 120.34(2 \mathrm{C}, \mathrm{s}, \mathrm{C}-$ 3,5), $114.84\left(\mathrm{t},{ }^{1} \mathrm{~J}_{\mathrm{C}-\mathrm{F}}=244.4 \mathrm{~Hz}, \mathrm{CHF}_{2}\right), 12.38(\mathrm{~s}, \mathrm{Me}) \mathrm{ppm}$. HRMS $(E S I+)$ for $\mathrm{C}_{10} \mathrm{H}_{9} \mathrm{~F}_{5} \mathrm{NO}[\mathrm{M}+\mathrm{H}]$ : calcd 254.0599, found 254.0622 .

3-N-(1,1-Difluoropropan-2-ylidene)-1- $\mathrm{N}, \mathrm{N}$-dimethylbenzene-

1,3-diamine 1kk. Under argon atmosphere, an excess of cold 1,1-difluoroacetone (2 equiv., $0.83 \mathrm{~mL}, 10.2 \mathrm{mmol}$ ) was added to $1-N, N$-dimethyl-1,3-phenylenediamine dihydrochloride (1 equiv., $695 \mathrm{mg}, 5.1 \mathrm{mmol}$ ) and sodium hydride (2.1 equiv., $257.2 \mathrm{mg}, 10.72 \mathrm{mmol})$ in anhydrous DCM $(15 \mathrm{~mL})$ in presence of ( $4 \AA \mathrm{MS})$. Then the reaction mixture was stirred for $24 \mathrm{~h}$ at room temperature. ( $4 \AA \mathrm{MS}$ ) was then filtered off on celite and washed with ether. The filtrate was concentrated under reduced pressure to give the 3-N-(1,1-Difluoropropan-2ylidene)-1- $N, N$-dimethylbenzene-1,3-diamine $1 \mathrm{kk}$ as a black paste $(1.26 \mathrm{~g}) .{ }^{1} \mathrm{H} N M R \delta \mathrm{H}=7.21\left(1 \mathrm{H}, \mathrm{t},{ }^{3} \mathrm{~J}_{\mathrm{H}-\mathrm{H}}=8.2 \mathrm{~Hz}, \mathrm{C}(5) \mathrm{H}\right)$, $6.56-6.51(1 \mathrm{H}, \mathrm{m}, \mathrm{C}(4) \mathrm{H}), 6.13-6.08(2 \mathrm{H}, \mathrm{m}, \mathrm{C}(2,6) \mathrm{H}), 6.06$ $\left(1 \mathrm{H}, \mathrm{t},{ }^{2} \mathrm{~J}_{\mathrm{H}-\mathrm{F}}=55.5 \mathrm{~Hz}, \mathrm{CHF}_{2}\right), 2.95\left(6 \mathrm{H}, \mathrm{s}, \mathrm{NMe}_{2}\right), 1.96(3 \mathrm{H}, \mathrm{s}$, Me) ppm. ${ }^{19} \mathrm{~F} \mathrm{NMR} \delta \mathrm{F}=-121.31\left(2 \mathrm{~F}, \mathrm{~d},{ }^{2} \mathrm{~J}_{\mathrm{F}-\mathrm{H}}=55.5 \mathrm{~Hz}, \mathrm{CHF}_{2}\right)$ ppm. HRMS $(E S I+)$ for $\mathrm{C}_{11} \mathrm{H}_{15} \mathrm{~F}_{2} \mathrm{~N}_{2}[\mathrm{M}+\mathrm{H}]$ : calcd 213.1198, found 213.1209.

4-N-(1,1-Difluoropropan-2-ylidene)-1- $\mathrm{N}, \mathrm{N}$-dimethylbenzene-

1,4-diamine 1Il. The product was prepared according to the general procedure and starting from 1,1-difluoroacetone (2 equiv., $1.19 \mathrm{~mL}, 14.7 \mathrm{mmol}$ ) and $\mathrm{N}, \mathrm{N}$-dimethyl-1,4benzenediamine (1 equiv., $0.92 \mathrm{~mL}, 7.35 \mathrm{mmol}$ ) in presence of (4 $\AA \mathrm{MS})$. The reaction mixture was stirred for $20 \mathrm{~h}$ at room temperature. 4-N-(1,1-Difluoropropan-2-ylidene)-1- $N, N$ dimethylbenzene-1,4-diamine 1 ll was provided as a yellow solid (1.46 g, 94\%). ${ }^{1} \mathrm{H}$ NMR $\delta \mathrm{H}=6.77\left(4 \mathrm{H}, \mathbf{A}_{2} \mathbf{B}_{2},{ }^{3} J_{\mathrm{H}-\mathrm{H}}=8.9 \mathrm{~Hz}\right.$, $\Delta v=27.76 \mathrm{~Hz}, \mathrm{C}(2,6 / 3,5) \mathrm{H}), 6.05\left(1 \mathrm{H}, \mathrm{t},{ }^{2} J_{\mathrm{H}-\mathrm{F}}=55.9 \mathrm{~Hz}, \mathrm{CHF}_{2}\right)$, $2.96\left(6 \mathrm{H}, \mathrm{s}, \mathrm{NMe}_{2}\right), 2.03(3 \mathrm{H}, \mathrm{s}, \mathrm{Me}) \mathrm{ppm} .{ }^{19} \mathrm{~F} \mathrm{NMR} \delta \mathrm{F}=-$ $120.62\left(2 \mathrm{~F}, \mathrm{~d},{ }^{2} J_{F-H}=55.9 \mathrm{~Hz}, \mathrm{CHF}_{2}\right) \mathrm{ppm} .{ }^{13} \mathrm{C} \mathrm{NMR} \delta \mathrm{C}=161.26$ $\left(\mathrm{t},{ }^{2} J_{\mathrm{C}-\mathrm{F}}=28.4 \mathrm{~Hz}, \mathrm{CCHF}_{2}\right), 148.61(\mathrm{~s}, \mathrm{C}-2), 137.60(\mathrm{~s}, \mathrm{C}-1)$, $121.72(2 \mathrm{C}, \mathrm{s}, \mathrm{C}-2,6), 115.70\left(\mathrm{t},{ }^{1} \mathrm{~J}_{\mathrm{C}-\mathrm{F}}=242.6 \mathrm{~Hz}, \mathrm{CHF}_{2}\right.$ ), 112.84 (2 C, s, C-3,5), 40.83 (2 C, s, NMe $)_{2}, 12.74$ (s, Me) ppm. HRMS $(\mathrm{ESI}+)$ for $\mathrm{C}_{11} \mathrm{H}_{15} \mathrm{~F}_{2} \mathrm{~N}_{2}[\mathrm{M}+\mathrm{H}]$ : calcd 213.1198, found 213.1214. $\mathrm{C}_{11} \mathrm{H}_{14} \mathrm{~F}_{2} \mathrm{~N}_{2}$ (212): calcd (\%) $\mathrm{N} 13.19, \mathrm{C} 62.19, \mathrm{H} 6.60$, found $\mathrm{N}$ 13.22, C 61.99, H 6.68. MP : $80.5-82^{\circ} \mathrm{C}$.

$\boldsymbol{N}$-(1,1-Difluoropropan-2-ylidene)-3-fluoro-2-methylaniline

$1 \mathbf{m m}$. The product was prepared according to the general 
procedure and starting from 1,1-difluoroacetone (2 equiv., $2.59 \mathrm{~mL}, 32 \mathrm{mmol}$ ) and 3-fluoro-2-methylaniline (1 equiv., 1.82 $\mathrm{mL}, 16 \mathrm{mmol}$ ) in presence of (4 $\AA \mathrm{MS})$. The reaction mixture was stirred for $18 \mathrm{~h}$ at room temperature. $\mathrm{N}-(1,1-$ Difluoropropan-2-ylidene)-3-fluoro-2-methylaniline $1 \mathrm{~mm}$ was provided as a brown oil ( $2.88 \mathrm{~g}, 90 \%$, estimated). ${ }^{1} \mathrm{H} \mathrm{NMR} \delta \mathrm{H}=$ $7.16-7.13(1 \mathrm{H}, \mathrm{m}, \mathrm{C}(4) \mathrm{H}), 6.83\left(1 \mathrm{H}, \mathrm{t},{ }^{3} \mathrm{~J}_{\mathrm{H}-\mathrm{H}}=8.8 \mathrm{~Hz}, \mathrm{C}(5) \mathrm{H}\right)$, $6.41\left(1 \mathrm{H}, \mathrm{d}, 3 \mathrm{~J}_{\mathrm{H}-\mathrm{H}}=7.9 \mathrm{~Hz}, \mathrm{C}(6) \mathrm{H}\right), 6.10\left(1 \mathrm{H}, \mathrm{t},{ }^{2} \mathrm{~J}_{\mathrm{H}-\mathrm{F}}=55.5 \mathrm{~Hz}\right.$, $\left.\mathrm{CHF}_{2}\right), 1.99\left(3 \mathrm{H}, \mathrm{d},{ }^{4} J_{H-F}=2.0 \mathrm{~Hz}, \mathrm{C}(2 \mathrm{Me}) \mathrm{H}\right), 1.89(3 \mathrm{H}, \mathrm{s}, \mathrm{Me})$ ppm. ${ }^{19} \mathrm{~F} N M R \delta F=-116.18--116.24(1 \mathrm{~F}, \mathrm{~m}, \mathrm{~F}),-121.21(2 \mathrm{~F}$, $\left.\mathrm{dd},{ }^{2} J_{F-H}=55.5,4 J_{F-H}=2.4 \mathrm{~Hz}, \mathrm{CHF}_{2}\right) \mathrm{ppm} .{ }^{13} \mathrm{C} \mathrm{NMR} \delta \mathrm{C}=164.50$ $\left(\mathrm{t},{ }^{2} J_{C-F}=29.0 \mathrm{~Hz}, C C F_{2}\right), 161.79\left(\mathrm{~d},{ }^{1} J_{C-F}=244.3 \mathrm{~Hz}, \mathrm{C}-3\right)$, $148.91\left(\mathrm{~d},{ }^{3} J_{C-F}=7.8 \mathrm{~Hz}, \mathrm{C}-1\right), 126.98\left(\mathrm{~d},{ }^{2} J_{C-F}=9.8 \mathrm{~Hz}, \mathrm{C}-4\right)$, $114.68(\mathrm{~s}, \mathrm{C}-2), 114.55\left(\mathrm{t},{ }^{1} \mathrm{~J}_{\mathrm{C}-F}=243.0 \mathrm{~Hz}, \mathrm{CHF}_{2}\right), 113.50\left(\mathrm{~d},{ }^{4} J_{C-F}\right.$ $=3.1 \mathrm{~Hz}, \mathrm{C}-6), 111.17$ (d, $\left.{ }^{3} \mathrm{~J}_{\mathrm{C}-F}=23.0 \mathrm{~Hz}, \mathrm{C}-5\right), 12.83(\mathrm{~s}, \mathrm{Me})$, $9.02\left(\mathrm{~d},{ }^{3} J_{C-F}=4.8 \mathrm{~Hz}, \mathrm{C}(2) \mathrm{Me}\right) \mathrm{ppm}$. HRMS $(\mathrm{ESI}+)$ for $\mathrm{C}_{10} \mathrm{H}_{11} \mathrm{~F}_{3} \mathrm{~N}$ $[\mathrm{M}+\mathrm{H}]$ : calcd 202.0838, found 202.0866.

\section{3-Chloro- $\mathbf{N}$-(1,1-difluoropropan-2-ylidene)-2-methylaniline}

1nn. The product was prepared according to the general procedure and starting from 1,1-difluoroacetone (2 equiv., $2.29 \mathrm{~mL}, 28.2 \mathrm{mmol}$ ) and 3-chloro-2-methylaniline (1 equiv., $1.71 \mathrm{~mL}, 14.1 \mathrm{mmol}$ ) in presence of (4 $\AA \mathrm{MS})$. The reaction mixture was stirred for $18 \mathrm{~h}$ at room temperature. 3-Chloro- $\mathrm{N}$ (1,1-difluoropropan-2-ylidene)-2-methylaniline 1nn was provided as a brown oil ( $2.79 \mathrm{~g}, 91 \%$, estimated). ${ }^{1} \mathrm{H} \mathrm{NMR} \delta \mathrm{H}=$ $7.17\left(1 \mathrm{H}, \mathrm{dd},{ }^{3} \mathrm{~J}_{\mathrm{H}-\mathrm{H}}=8.0,{ }^{4} \mathrm{~J}_{\mathrm{H}-\mathrm{H}}=1.1 \mathrm{~Hz}, \mathrm{C}(4) \mathrm{H}\right), 7.10\left(1 \mathrm{H}, \mathrm{t},{ }^{3} \mathrm{~J}_{\mathrm{H}-\mathrm{H}}\right.$ $=7.7 \mathrm{~Hz}, \mathrm{C}(5) \mathrm{H}), 6.52\left(1 \mathrm{H}, \mathrm{dd}, 3^{3} \mathrm{H}_{-H}=7.7,{ }^{4} J_{\mathrm{H}-\mathrm{H}}=0.9 \mathrm{~Hz}, \mathrm{C}(6) \mathrm{H}\right)$, $6.11\left(1 \mathrm{H}, \mathrm{t},{ }^{2} \mathrm{~J}_{\mathrm{H}-\mathrm{F}}=55.5 \mathrm{~Hz}, \mathrm{CHF}_{2}\right), 2.12(3 \mathrm{H}, \mathrm{s}, \mathrm{C}(2 \mathrm{Me}) \mathrm{H}), 1.88$ $(3 \mathrm{H}, \mathrm{s}, \mathrm{Me}) \mathrm{ppm} .{ }^{19} \mathrm{~F} \mathrm{NMR} \delta \mathrm{F}=-121.15\left(2 \mathrm{~F}, \mathrm{~d},{ }^{2} \mathrm{~J}_{\mathrm{F}-\mathrm{H}}=55.5 \mathrm{~Hz}\right.$, $\left.\mathrm{CHF}_{2}\right) \mathrm{ppm} .{ }^{13} \mathrm{C} N M R \delta \mathrm{C}=164.43\left(\mathrm{t},{ }^{2} \mathrm{~J}_{\mathrm{C}-\mathrm{F}}=28.9 \mathrm{~Hz}, \mathrm{CCHF}_{2}\right)$, 148.44 (s, C-1), 135.57 (s, C-3), 127.06 (s, C-5), 125.48 (s, C-4), $116.39(\mathrm{~s}, \mathrm{C}-6), 114.63\left(\mathrm{t},{ }^{1} \mathrm{~J}_{\mathrm{C}-F}=243.1 \mathrm{~Hz}, \mathrm{CHF}_{2}\right.$ ), 113.56 (s, C2), 14.26 (s, C(2)Me, 12.89 (s, Me) ppm. HRMS (ESI +) for $\mathrm{C}_{10} \mathrm{H}_{11} \mathrm{~F}_{2} \mathrm{NCl}[\mathrm{M}+\mathrm{H}]$ : calcd 218.0543, found 218.0557 .

3-Chloro- $\mathbf{N}$-(1,1-difluoropropan-2-ylidene)aniline 100 . The product was prepared according to the general procedure and starting from 1,1-difluoroacetone (2 equiv., $5.08 \mathrm{~mL}, 62.7$ mmol) and 3-chloroaniline (1 equiv., $3.33 \mathrm{~mL}, 31.4 \mathrm{mmol}$ ) in presence of ( $4 \AA \mathrm{MS})$. The reaction mixture was stirred for $18 \mathrm{~h}$ at room temperature. 3-Chloro- $N$-(1,1-difluoropropan-2ylidene)-aniline 100 was provided as a brown oil $(9.68 \mathrm{~g}) .{ }^{1} \mathrm{H}$ $\mathrm{NMR} \delta \mathrm{H}=7.31\left(1 \mathrm{H}, \mathrm{t},{ }^{3} \mathrm{~J}_{\mathrm{H}-\mathrm{H}}=8.0 \mathrm{~Hz}, \mathrm{C}(5) \mathrm{H}\right), 7.16\left(1 \mathrm{H}, \mathrm{d},{ }^{3} \mathrm{~J}_{\mathrm{H}-\mathrm{H}}=\right.$ $8.1 \mathrm{~Hz}, \mathrm{C}(4) \mathrm{H}), 6.81\left(1 \mathrm{H}, \mathrm{t},{ }^{4} J_{\mathrm{H}-H}=1.9 \mathrm{~Hz}, \mathrm{C}(2) \mathrm{H}\right), 6.68(1 \mathrm{H}, \mathrm{d}$, $\left.{ }^{3} J_{H-H}=7.9 \mathrm{~Hz}, \mathrm{C}(6) \mathrm{H}\right), 6.06\left(1 \mathrm{H}, \mathrm{t},{ }^{2} J_{H-F}=55.4 \mathrm{~Hz}, \mathrm{CHF}_{2}\right), 1.97$ (3 $\mathrm{H}, \mathrm{s}, \mathrm{Me}) \mathrm{ppm} .{ }^{19} \mathrm{~F}$ NMR $\delta \mathrm{F}=-121.46\left(2 \mathrm{~F}, \mathrm{~d},{ }^{2} J_{F-H}=55.4 \mathrm{~Hz}\right.$, $\left.\mathrm{CHF}_{2}\right) \mathrm{ppm} .{ }^{13} \mathrm{C} N M R \delta \mathrm{C}=164.76\left(\mathrm{t},{ }^{2} \mathrm{~J}_{\mathrm{C}-\mathrm{F}}=29.0 \mathrm{~Hz}, \mathrm{CCHF}_{2}\right)$, 149.64 (s, C-1), 134.99 (s, C-3), 130.44 (s, C-5), 124.96 (s, C-4), $119.18(\mathrm{~s}, \mathrm{C}-2), 117.24(\mathrm{~s}, \mathrm{C}-6), 114.55\left(\mathrm{t},{ }^{1} \mathrm{~J}_{\mathrm{C}-\mathrm{F}}=243.2 \mathrm{~Hz}\right.$, $\mathrm{CHF}_{2}$ ), 12.87 (s, Me) ppm. HRMS (ESI +) for $\mathrm{C}_{9} \mathrm{H}_{9} \mathrm{~F}_{2} \mathrm{NCl}[\mathrm{M}+\mathrm{H}]$ : calcd 204.0386, found 204.0367.

\section{$\mathbf{N}$-(1,1-Difluoropropan-2-ylidene)-4-(trifluoromethyl)aniline}

1pp. The product was prepared according to the general procedure and starting from 1,1-difluoroacetone (2 equiv., $0.503 \mathrm{~mL}, 6.21 \mathrm{mmol}$ ) and 4-(trifluoromethyl)aniline (1 equiv., $0.386 \mathrm{~mL}, 3.1 \mathrm{mmol}$ ) in presence of anhydrous $\mathrm{MgSO}_{4}$. The reaction mixture was stirred for $16 \mathrm{~h}$ at room temperature. $\mathrm{N}$ (1,1-Difluoropropan-2-ylidene)-4-(trifluoromethyl)aniline 1pp was provided as a brown oil (212 $\mathrm{mg}, 29 \%$, estimated). ${ }^{1} \mathrm{H} \delta \mathrm{H}=$ $7.63\left(2 \mathrm{H}, \mathrm{d},{ }^{3} J_{\mathrm{H}-\mathrm{H}}=8.3 \mathrm{~Hz}, \mathrm{C}(3,5) \mathrm{H}\right), 6.85\left(2 \mathrm{H}, \mathrm{d},{ }^{3} J_{\mathrm{H}-\mathrm{H}}=8.2 \mathrm{~Hz}\right.$, $\mathrm{C}(2,6) \mathrm{H}), 6.05\left(1 \mathrm{H}, \mathrm{t},{ }^{2} J_{\mathrm{H}-\mathrm{F}}=55.4 \mathrm{~Hz}, \mathrm{CHF}_{2}\right), 1.93(3 \mathrm{H}, \mathrm{s}, \mathrm{Me})$ ppm. ${ }^{19} \mathrm{~F} \mathrm{NMR} \delta \mathrm{F}=-62.13\left(3 \mathrm{~F}, \mathrm{~s}, \mathrm{CF}_{3}\right),-121.34--121.59(2 \mathrm{~F}$, $\mathrm{m}, \mathrm{CHF}_{2}$ ) $)$ ppm. ${ }^{13} \mathrm{C} \mathrm{NMR} \delta \mathrm{C}=164.59\left(\mathrm{t},{ }^{2} \mathrm{~J}_{\mathrm{C}-\mathrm{F}}=29.0 \mathrm{~Hz}, \mathrm{CCHF}_{2}\right)$, 151.64 (s, C-1), $126.64\left(2 \mathrm{C}, \mathrm{q},{ }^{3} J_{C-F}=3.8 \mathrm{~Hz}, \mathrm{C}-3,5\right), 124.30$ (q, $\left.{ }^{1} J_{C-F}=271.6 \mathrm{~Hz}, \mathrm{CF}_{3}\right), 120.30\left(\mathrm{q},{ }^{2} J_{C-F}=32.6 \mathrm{~Hz}, \mathrm{C}-4\right), 119.03(2$ C, s, C-2,6), $114.49\left(\mathrm{t},{ }^{1} J_{C-F}=243.3 \mathrm{~Hz}, \mathrm{CHF}_{2}\right), 12.97$ (s, Me) ppm. HRMS $(E S I+)$ for $\mathrm{C}_{10} \mathrm{H}_{9} \mathrm{~F}_{5} \mathrm{~N}[\mathrm{M}+\mathrm{H}]$ : calcd 238.0650, found 238.0658 .

\section{$\mathbf{N}$-(1,1-Difluoropropan-2-ylidene)-3-(trifluoromethyl)aniline}

1qq. The product was prepared according to the general procedure and starting from 1,1-difluoroacetone (2 equiv., $1.01 \mathrm{~mL}, 12.4 \mathrm{mmol}$ ) and 3-(trifluoromethyl)aniline (1 equiv., $0.767 \mathrm{~mL}, 6.21 \mathrm{mmol}$ ) in presence of (4 ̊ MS). The reaction mixture was stirred for $5 \mathrm{~h}$ at room temperature. $\mathrm{N}$-(1,1Difluoropropan-2-ylidene)-3-(trifluoromethyl)aniline 1qq was provided as a brown oil $\left(1.47 \mathrm{~g}, 93 \%\right.$, estimated). ${ }^{1} \mathrm{H} \mathrm{NMR} \delta \mathrm{H}=$ $7.49\left(1 \mathrm{H}, \mathrm{t},{ }^{3} \mathrm{~J}_{\mathrm{H}-\mathrm{H}}=7.8 \mathrm{~Hz}, \mathrm{C}(5) \mathrm{H}\right), 7.42\left(1 \mathrm{H}, \mathrm{d},{ }^{3} \mathrm{~J}_{\mathrm{H}-\mathrm{H}}=7.8 \mathrm{~Hz}\right.$, $\mathrm{C}(4) \mathrm{H}), 7.03(1 \mathrm{H}, \mathrm{s}, \mathrm{C}(2) \mathrm{H}), 6.94\left(1 \mathrm{H}, \mathrm{d}, 3^{3} \mathrm{H}_{-H}=7.2 \mathrm{~Hz}, \mathrm{C}(4) \mathrm{H}\right)$, $6.05\left(1 \mathrm{H}, \mathrm{t},{ }^{2} \mathrm{~J}_{\mathrm{H}-\mathrm{F}}=55.5 \mathrm{~Hz}, \mathrm{CHF}_{2}\right), 1.95(3 \mathrm{H}, \mathrm{s}, \mathrm{Me}) \mathrm{ppm} .{ }^{19} \mathrm{~F}$ NMR $\delta F=-62.80\left(3 \mathrm{~F}, \mathrm{~s}, \mathrm{CF}_{3}\right),-121.44\left(2 \mathrm{~F}, \mathrm{~d}^{2} \mathrm{~J}_{F-H}=55.4 \mathrm{~Hz}\right.$, $\left.\mathrm{CHF}_{2}\right) \mathrm{ppm} .{ }^{13} \mathrm{C} \mathrm{NMR} \delta \mathrm{C}=165.21\left(\mathrm{t},{ }^{2} \mathrm{~J}_{\mathrm{C}-\mathrm{F}}=28.9 \mathrm{~Hz},\left(\mathrm{CCHF}_{2}\right)\right.$, $148.91(\mathrm{~s}, \mathrm{C}-1), 131.84$ (q, $\left.{ }^{2} J_{C-F}=32.5 \mathrm{~Hz}, \mathrm{C}-3\right), 129.96(\mathrm{~s}, \mathrm{C}-5)$, $124.03\left(\mathrm{q},{ }^{1} J_{C-F}=272.3 \mathrm{~Hz}, \mathrm{CF}_{3}\right), 122.38(\mathrm{~s}, \mathrm{C}-6), 121.68\left(\mathrm{q},{ }^{3} J_{C-F}\right.$ $=3.8 \mathrm{~Hz}, \mathrm{C}-4), 116.01\left(\mathrm{q},{ }^{3} \mathrm{~J}_{\mathrm{C}-\mathrm{F}}=4.0 \mathrm{~Hz}, \mathrm{C}-2\right), 114.53\left(\mathrm{t},{ }^{1} J_{\mathrm{C}-F}=\right.$ $\left.243.2 \mathrm{~Hz}, \mathrm{CHF}_{2}\right), 12.78(\mathrm{~s}, \mathrm{Me}) \mathrm{ppm}$. HRMS (ESI +) for $\mathrm{C}_{10} \mathrm{H}_{9} \mathrm{~F}_{5} \mathrm{~N}$ $[\mathrm{M}+\mathrm{H}]$ : calcd 238.0650, found 238.0660.

$\mathbf{N}$-(1,1-Difluoropropan-2-ylidene)-2-(trifluoromethyl)aniline

1rr. The product was prepared according to the general procedure and starting from 1,1-difluoroacetone (2 equiv., $0.503 \mathrm{~mL}, 6.21 \mathrm{mmol}$ ) and 2-(trifluoromethyl)aniline (1 equiv., $0.386 \mathrm{~mL}, 3.1 \mathrm{mmol}$ ) in presence of anhydrous $\mathrm{MgSO}_{4}$. The reaction mixture was stirred for $16 \mathrm{~h}$ at room temperature. $\mathrm{N}$ (1,1-Difluoropropan-2-ylidene)-2-(trifluoromethyl)aniline $\mathbf{1 r r}$ was provided as a brown oil (291.5 mg, 40\%, estimated). ${ }^{1} \mathrm{H}$ NMR $\delta \mathrm{H}=7.67\left(1 \mathrm{H}, \mathrm{d},{ }^{3} \mathrm{~J}_{\mathrm{H}-\mathrm{H}}=7.9 \mathrm{~Hz}, \mathrm{C}(3) \mathrm{H}\right), 7.52\left(1 \mathrm{H}, \mathrm{t},{ }^{3} \mathrm{~J}_{\mathrm{H}-\mathrm{H}}=\right.$ $7.7 \mathrm{~Hz}, \mathrm{C}(5) \mathrm{H}), 7.23\left(1 \mathrm{H}, \mathrm{t},{ }^{3} \mathrm{~J}_{\mathrm{H}-\mathrm{H}}=7.7 \mathrm{~Hz}, \mathrm{C}(5) \mathrm{H}\right), 6.72(1 \mathrm{H}, \mathrm{d}$, $\left.{ }^{3} J_{H-H}=7.9 \mathrm{~Hz}, \mathrm{C}(6) \mathrm{H}\right), 6.08\left(1 \mathrm{H}, \mathrm{t},{ }^{2} \mathrm{~J}_{\mathrm{H}-\mathrm{F}}=55.4 \mathrm{~Hz}, \mathrm{CHF}_{2}\right), 1.90(3$ $\mathrm{H}, \mathrm{s}, \mathrm{Me}) \mathrm{ppm} .{ }^{19} \mathrm{~F} N M R \delta \mathrm{F}=-61.85\left(3 \mathrm{~F}, \mathrm{~s}, \mathrm{CF}_{3}\right),-121.82(2 \mathrm{~F}, \mathrm{~d}$, $\left.{ }^{2} J_{F-H}=55.4 \mathrm{~Hz}, \mathrm{CHF}_{2}\right) \mathrm{ppm} .{ }^{13} \mathrm{C} \mathrm{NMR} \delta \mathrm{C}=165.58\left(\mathrm{t},{ }^{2} \mathrm{~J}_{\mathrm{C}-\mathrm{F}}=29.4\right.$ $\left.\mathrm{Hz}, \mathrm{CCHF}_{2}\right), 146.98\left(\mathrm{q},{ }^{3} \mathrm{~J}_{\mathrm{C}-F}=2.02 \mathrm{~Hz}, \mathrm{C}-1\right), 132.87(\mathrm{~s}, \mathrm{C}-5)$, $126.77\left(q,{ }^{3} J_{C-F}=5.05 \mathrm{~Hz}, \mathrm{C}-3\right), 124.50(\mathrm{~s}, \mathrm{C}-4), 123.67\left(\mathrm{q},{ }^{1} J_{C-F}=\right.$ $\left.273.0 \mathrm{~Hz}, \mathrm{CF}_{3}\right), 119.65\left(\mathrm{q},{ }^{2} \mathrm{~J}_{\mathrm{C}-\mathrm{F}}=31.3 \mathrm{~Hz}, \mathrm{C}-2\right), 119.18$ (s, C-6), $114.27\left(\mathrm{t},{ }^{1} J_{C-F}=243.3 \mathrm{~Hz}, \mathrm{CHF}_{2}\right), 13.42(\mathrm{~s}, \mathrm{Me}) \mathrm{ppm}$. HRMS (ESI +) for $\mathrm{C}_{10} \mathrm{H}_{9} \mathrm{~F}_{5} \mathrm{~N}[\mathrm{M}+\mathrm{H}]$ : calcd 238.0650, found 238.0654 .

$\mathbf{N}$-(1,1-Difluoropropan-2-ylidene)-2-phenylaniline 1ss. The product was prepared according to the general procedure and starting from 1,1-difluoroacetone (2 equiv., $0.485 \mathrm{~mL}, 5.98$ $\mathrm{mmol}$ ) and 2-aminodiphenyl (1 equiv., $505 \mathrm{mg}, 2.99 \mathrm{mmol}$ ) in presence of anhydrous $\mathrm{MgSO}_{4}$. The reaction mixture was stirred for $14 \mathrm{~h}$ at room temperature. $\mathrm{N}$-(1,1-Difluoropropan-2ylidene)-2-phenylaniline 1ss was provided as a brown oil $(953.7 \mathrm{mg}) .{ }^{1} \mathrm{H} \quad \mathrm{NMR} \delta \mathrm{H}=7.42-7.23(8 \mathrm{H}, \mathrm{m}$, $\left.\mathrm{C}\left(3,4,5,2^{\prime}, 3^{\prime}, 4^{\prime}, 5^{\prime}, 6^{\prime}\right) \mathrm{H}\right), 6.71\left(1 \mathrm{H}, \mathrm{dd},{ }^{3} J_{H-H}=7.8,{ }^{4} J_{H-H}=1.0 \mathrm{~Hz}\right.$, $\mathrm{C}(6) \mathrm{H}), 5.88\left(1 \mathrm{H}, \mathrm{t},{ }^{2} \mathrm{~J}_{\mathrm{H}-\mathrm{F}}=55.6 \mathrm{~Hz}, \mathrm{CHF}_{2}\right), 1.64(3 \mathrm{H}, \mathrm{s}, \mathrm{Me})$ ppm. ${ }^{19} \mathrm{~F} N M R \delta F=-121.61\left(2 \mathrm{~F}, \mathrm{~d},{ }^{2} J_{F-H}=55.5 \mathrm{~Hz}, \mathrm{CHF}_{2}\right) \mathrm{ppm}$. 
${ }^{13} \mathrm{C} N M R \delta C=163.53\left(\mathrm{t},{ }^{2} \mathrm{~J}_{\mathrm{C}-\mathrm{F}}=28.8 \mathrm{~Hz}, \mathrm{CCHF}_{2}\right), 146.07(\mathrm{~s}, \mathrm{C}-1)$, 139.00 (s, C-1'), 132.02 (s, C-2), $131.44-123.83$ (8 C, m, C3,4,5, 2', 3', 4',5',6'), $119.16(\mathrm{~s}, \mathrm{C}-6), 114.67\left(\mathrm{t},{ }^{1} J_{C-F}=243.2 \mathrm{~Hz}\right.$, $\mathrm{CHF}_{2}$ ), 13.40 (s, Me) ppm. HRMS (ESI positive) for $\mathrm{C}_{15} \mathrm{H}_{14} \mathrm{~F}_{2} \mathrm{~N}[\mathrm{M}$ $+\mathrm{H}$ ]: calcd 246.1089, found 246.1112 .

\section{$\boldsymbol{N}$-(1,1-Difluoropropan-2-ylidene)-2-(phenylsulfanyl)aniline}

1tt. The product was prepared according to the general procedure and starting from 1,1-difluoroacetone (2 equiv., $0.422 \mathrm{~mL}, 5.2 \mathrm{mmol}$ ) and 2-(phenylsulfanyl)aniline (1 equiv., $523 \mathrm{mg}, 2.6 \mathrm{mmol}$ ) in presence of anhydrous $\mathrm{MgSO}_{4}$. The reaction mixture was stirred for $12 \mathrm{~h}$ at room temperature. $\mathrm{N}$ (1,1-Difluoropropan-2-ylidene)-2-(phenylsulfanyl)aniline 1tt was provided as a brown oil (796.1 mg). ${ }^{1} \mathrm{H} \mathrm{NMR} \delta \mathrm{H}=7.34-$ $7.20\left(6 \mathrm{H}, \mathrm{m}, \mathrm{C}\left(3,2^{\prime}, 3^{\prime}, 4^{\prime}, 5^{\prime}, 6^{\prime}\right) \mathrm{H}\right), 7.09\left(2 \mathrm{H}, \mathrm{td},{ }^{3} J_{H-H}=7.65,{ }^{4} J_{H-H}\right.$ $=1.3 \mathrm{~Hz}, \mathrm{C}(4,5) \mathrm{H}), 6.69\left(1 \mathrm{H}, \mathrm{dd},{ }^{3} J_{\mathrm{H}-\mathrm{H}}=7.8,4 J_{H-H}=1.1 \mathrm{~Hz}\right.$, $\mathrm{C}(6) \mathrm{H}), 6.01\left(1 \mathrm{H}, \mathrm{t},{ }^{2} \mathrm{~J}_{\mathrm{H}-\mathrm{F}}=55.5 \mathrm{~Hz}, \mathrm{CHF}_{2}\right), 1.78(3 \mathrm{H}, \mathrm{s}, \mathrm{Me})$ ppm. ${ }^{19} \mathrm{~F} N M R \delta F=-121.06\left(2 \mathrm{~F}, \mathrm{~d},{ }^{2} J_{F-H}=55.4 \mathrm{~Hz}, \mathrm{CHF}_{2}\right) \mathrm{ppm}$. ${ }^{13} \mathrm{C} \mathrm{NMR} \delta \mathrm{C}=164.78\left(\mathrm{t},{ }^{2} \mathrm{~J}_{\mathrm{C}-\mathrm{F}}=28.9 \mathrm{~Hz}, \mathrm{CCHF}_{2}\right), 148.85(\mathrm{~s}, \mathrm{C}-1)$, $136.88\left(\mathrm{~s}, \mathrm{C}-1^{\prime}\right), 134.64(\mathrm{~s}, \mathrm{C}-2), 132.37-127.42$ (6 C, m, C3,2', 3', 4', 5', 6'), 126.57 (s, C-5), 125.43 (s, C-4), 118.87 (s, C-6), $114.45\left(\mathrm{t},{ }^{1} J_{C-F}=243.3 \mathrm{~Hz}, \mathrm{CHF}_{2}\right.$ ), 13.04 (s, Me) ppm. HRMS (ESI + ) for $\mathrm{C}_{15} \mathrm{H}_{14} \mathrm{~F}_{2} \mathrm{NS}[\mathrm{M}+\mathrm{H}]$ : calcd 278.0810, found 278.0781.

5-[(1,1-Difluoropropan-2-ylidene)amino]-1-naphthol 1uu. The product was prepared according to the general procedure and starting from 1,1-difluoroacetone (2 equiv., $0.509 \mathrm{~mL}, 6.28$ $\mathrm{mmol}$ ) and 5-amino-1-naphthol (1 equiv., $500 \mathrm{mg}, 3.14 \mathrm{mmol}$ ) in presence of anhydrous $\mathrm{MgSO}_{4}$. The reaction mixture was stirred for $14 \mathrm{~h}$ at room temperature. 5-[(1,1-Difluoropropan2-ylidene)amino]-1-naphthol 1uu was provided as a purple solid (670 mg, 91\%, estimated). ${ }^{1} \mathrm{H} N M R \delta H=8.01\left(1 \mathrm{H}, \mathrm{d}^{3}{ }^{3} \mathrm{H}-\mathrm{H}\right.$ $=8.5 \mathrm{~Hz}, \mathrm{C}(8) \mathrm{H}), 7.45\left(1 \mathrm{H}, \mathrm{t},{ }^{3} \mathrm{~J}_{\mathrm{H}-\mathrm{H}}=8.0 \mathrm{~Hz}, \mathrm{C}(7) \mathrm{H}\right), 7.35-7.17$ $(2 \mathrm{H}, \mathrm{m}, \mathrm{C}(3,4) \mathrm{H}), 6.84\left(1 \mathrm{H}, \mathrm{d},{ }^{3} J_{\mathrm{H}-\mathrm{H}}=7.3 \mathrm{~Hz}, \mathrm{C}(2) \mathrm{H}\right), 6.78(1 \mathrm{H}$, d, $\left.{ }^{3} J_{H-H}=7.2 \mathrm{~Hz}, \mathrm{C}(6) \mathrm{H}\right), 6.24\left(1 \mathrm{H}, \mathrm{t},{ }^{2} J_{H-F}=55.6 \mathrm{~Hz}, \mathrm{CHF}_{2}\right), 5.45$ $(1 \mathrm{H}, \mathrm{br} \mathrm{s}, \mathrm{OH}), 1.91(3 \mathrm{H}, \mathrm{s}, \mathrm{Me}) \mathrm{ppm} .{ }^{19} \mathrm{~F} \mathrm{NMR} \delta \mathrm{F}=-120.84(2$ $\left.\mathrm{F}, \mathrm{d},{ }^{2} J_{F-H}=55.5 \mathrm{~Hz}, \mathrm{CHF}_{2}\right) \mathrm{ppm} .{ }^{13} \mathrm{C} \mathrm{NMR} \delta \mathrm{C}=165.18\left(\mathrm{t},{ }^{2} J_{\mathrm{C}-F}=\right.$ $28.9 \mathrm{~Hz}, \mathrm{CCHF}_{2}$ ), 151.81 (s, C-1), 144.46 (s, C-5), 126.46 (s, C-1C-C-8), 126.24 (s, C-3), 125.23 (s, C-4-C-C-5), 125.00 (s, C-7), 118.80 (s, C-8), 115.48 (s, C-4), $114.83\left(\mathrm{t},{ }^{1} \mathrm{~J}_{\mathrm{C}-\mathrm{F}}=243.3 \mathrm{~Hz}\right.$, $\mathrm{CHF}_{2}$ ), 114.07 (s, C-6), 109.46 (s, C-2), 13.18 (s, Me) ppm. HRMS $\left(\mathrm{ESI}+\right.$ ) for $\mathrm{C}_{13} \mathrm{H}_{12} \mathrm{~F}_{2} \mathrm{NO}[\mathrm{M}+\mathrm{H}]$ : calcd 236.0881, found 236.0909.

General procedure for the synthesis of quinoline derivatives 2 and 3

Under argon atmosphere, a solution of the desired FAR (1,1,2,2-tetrafluoro- $N, N$-dimethylethan-1-amine (4a; TFEDMA), 2-chloro- $N, N$-diethyl-1,1,2-trifluoroethan-1-amine

(4b;

Yarovenko reagent), and $N, N$-diethyl-1,1,2,3,3,3hexafluoropropan-1-amine (4c; Ishikawa reagent)) (1.2 equiv.) was activated by adding boron trifluoride diethyl etherate $\left(\mathrm{BF}_{3} \bullet \mathrm{Et}_{2} \mathrm{O}\right)$ (1.2 equiv.) in dry acetonitrile $(3.6 \mathrm{mmol} / 5 \mathrm{~mL})$ and stirred for $15 \mathrm{~min}$. Then a solution of the desired ketimine derivative 1 (1 equiv.) in dry acetonitrile $(3 \mathrm{mmol} / 5 \mathrm{~mL}$ ) was slowly added to this mixture via syringe. After $15 \mathrm{~min}$ at room temperature, the mixture was heated at $50{ }^{\circ} \mathrm{C}$ for $19 \mathrm{~h}$. Acetonitrile was removed under reduced pressure and the reaction mixture was purified by flash chromatography using a gradient of ethyl acetate in pentane to provide the final compound $\mathbf{2}$ or $\mathbf{3}$.

4-(Difluoromethyl)-2-(trifluoromethyl)quinoline 2ai. The product was prepared according to the general procedure and starting from an activated solution of TFEDMA 4a (1.2 equiv., $0.42 \mathrm{~mL}, 3.61 \mathrm{mmol}$ ) by $\left(\mathrm{BF}_{3} \bullet \mathrm{Et}_{2} \mathrm{O}\right)$ (1.2 equiv., $0.46 \mathrm{~mL}, 3.61$ $\mathrm{mmol})$ and $\mathrm{N}$-(1,1,1-trifluoropropan-2-ylidene)aniline 1 (1 equiv., $562 \mathrm{mg}, 3.01 \mathrm{mmol}$ ) in presence of anhydrous acetonitrile. 4-(Difluoromethyl)-2-(trifluoromethyl)quinoline 2ai was provided after purification using a gradient of ethyl acetate in pentane (0-5\%) as a yellow solid (458 mg, 62\%). ${ }^{1} \mathrm{H}$ $\mathrm{NMR} \delta \mathrm{H}=8.33\left(1 \mathrm{H}, \mathrm{d},{ }^{3} \mathrm{~J}_{\mathrm{H}-\mathrm{H}}=8.5 \mathrm{~Hz}, \mathrm{C}(8) \mathrm{H}\right), 8.16\left(1 \mathrm{H}, \mathrm{d}, 3^{3} \mathrm{~J}_{\mathrm{H}-\mathrm{H}}\right.$ $=8.5 \mathrm{~Hz}, \mathrm{C}(5) \mathrm{H}), 7.93(1 \mathrm{H}, \mathrm{s}, \mathrm{C}(3) \mathrm{H}), 7.92-7.87(1 \mathrm{H}, \mathrm{m}$, $\mathrm{C}(7) \mathrm{H}), 7.80\left(1 \mathrm{H}, \mathrm{t}, 3_{\mathrm{H}-\mathrm{H}}=7.7 \mathrm{~Hz}, \mathrm{C}(6) \mathrm{H}\right), 7.22\left(1 \mathrm{H}, \mathrm{t},{ }^{2} J_{\mathrm{H}-\mathrm{F}}=\right.$ $\left.54.2 \mathrm{~Hz}, \mathrm{C}\left(4-\mathrm{CHF}_{2}\right) \mathrm{H}\right) \mathrm{ppm} .{ }^{19} \mathrm{~F} \mathrm{NMR} \delta \mathrm{F}=-67.66\left(3 \mathrm{~F}, \mathrm{~s}, \mathrm{CF}_{3}\right)$, $115.53\left(2 \mathrm{~F}, \mathrm{~d},{ }^{2} J_{F-H}=54.1 \mathrm{~Hz}, \mathrm{CHF}_{2}\right) \mathrm{ppm} .{ }^{13} \mathrm{C} \mathrm{NMR} \delta \mathrm{C}=147.94$ $\left(q,{ }^{2} J_{C-F}=35.3 \mathrm{~Hz}, \mathrm{C}-2\right), 147.87(\mathrm{t}, \mathrm{C}-8-\mathrm{C}-\mathrm{N}), 140.33\left(\mathrm{t},{ }^{2} J_{C-F}=\right.$ $22.3 \mathrm{~Hz}, \mathrm{C}-4), 131.39$ (s, C-7), 131.29 (s, C-8), 130.06 (s, C-6), 125.09 (s, C-5-C-C-4), 123.44 (s, C-5), 121.33 (q, ${ }^{1} J_{C-F}=275.73 \mathrm{~Hz}$, $\left.\mathrm{C}(2) \mathrm{CF}_{3}\right), 114.14\left(\mathrm{td},{ }^{3} \mathrm{~J}_{C-F}=7.9,{ }^{3} \mathrm{~J}_{C-F}=2.1 \mathrm{~Hz}, \mathrm{C}-3\right), 112.74\left(\mathrm{t},{ }^{1} \mathrm{~J}_{C-}\right.$ $\left.F=241.5 \mathrm{~Hz}, \mathrm{C}(4) \mathrm{CHF}_{2}\right)$ ppm. HRMS $\left(\mathrm{ESI}+\right.$ ) for $\mathrm{C}_{11} \mathrm{H}_{7} \mathrm{~F}_{5} \mathrm{~N}[\mathrm{M}+$ $\mathrm{H}$ ]: calcd 248.0493, found 248.0520. $\mathrm{C}_{11} \mathrm{H}_{6} \mathrm{~F}_{5} \mathrm{~N}$ (247): calcd (\%) N 5.66, C 53.40, H 2.43, found N 5.73, C 53.83, H 2.58. MP: 64 $-65.1^{\circ} \mathrm{C}$.

4-[Chloro(fluoro)methyl]-2-(trifluoromethyl)quinoline 2aii. The product was prepared according to the general procedure and starting from an activated solution of Yarovenko's reagent 4b (1.2 equiv., $0.73 \mathrm{~mL}, 3.21 \mathrm{mmol})$ by $\left(\mathrm{BF}_{3} \bullet \mathrm{Et}_{2} \mathrm{O}\right)$ ( 1.2 equiv., $0.41 \mathrm{~mL}, \quad 3.21 \mathrm{mmol})$ and $\mathrm{N}$-(1,1,1-trifluoropropan-2ylidene)aniline 1a (1 equiv., $500 \mathrm{mg}, 2.67 \mathrm{mmol}$ ) in presence of anhydrous acetonitrile. 4-[Chloro(fluoro)methyl]-2(trifluoromethyl)quinoline 2aii was provided after purification using a gradient of ethyl acetate in pentane (0-5\%) as a light yellow solid (402 mg, 57\%). ${ }^{1} \mathrm{H} \mathrm{NMR} \delta \mathrm{H}=8.32\left(1 \mathrm{H}, \mathrm{d},{ }^{3} \mathrm{~J}_{\mathrm{H}-\mathrm{H}}=\right.$ $8.4 \mathrm{~Hz}, \mathrm{C}(8) \mathrm{H}), 8.13\left(1 \mathrm{H}, \mathrm{d}, 3^{3} \mathrm{H}_{-H}=8.5 \mathrm{~Hz}, \mathrm{C}(5) \mathrm{H}\right), 7.95(1 \mathrm{H}, \mathrm{s}$, $\mathrm{C}(3) \mathrm{H}), 7.92-7.88(1 \mathrm{H}, \mathrm{m}, \mathrm{C}(7) \mathrm{H}), 7.81-7.77(1 \mathrm{H}, \mathrm{m}, \mathrm{C}(3) \mathrm{H})$, $7.66\left(1 \mathrm{H}, \mathrm{d},{ }^{2} J_{H-\mathrm{F}}=48.9 \mathrm{~Hz}, \mathrm{C}(4-\mathrm{CHFCl}) \mathrm{H}\right) \mathrm{ppm} .{ }^{19} \mathrm{~F} \mathrm{NMR} \delta \mathrm{F}=-$ $67.65\left(3 \mathrm{~F}, \mathrm{~s}, \mathrm{CF}_{3}\right),-138.75\left(1 \mathrm{~F}, \mathrm{~d}, 2 \mathrm{~J}_{F-H}=48.9 \mathrm{~Hz}, \mathrm{C} 4-\mathrm{CHFCl}\right)$ ppm. ${ }^{13} \mathrm{C}$ NMR $\delta \mathrm{C}=148.02\left(\mathrm{q},{ }^{2} J_{C-F}=35.4 \mathrm{~Hz}, \mathrm{C}-2\right), 147.88(\mathrm{~s}, \mathrm{C}-$ 8-C-N), $143.82\left(\mathrm{~d},{ }^{2} J_{C-F}=21.1 \mathrm{~Hz}, \mathrm{C}-4\right), 131.40(\mathrm{~s}, \mathrm{C}-7), 131.31$ (s, C-8), $129.82(\mathrm{~s}, \mathrm{C}-6), 124.03\left(\mathrm{~d},{ }^{2} J_{C-F}=3.6 \mathrm{~Hz}, \mathrm{C}-5-\mathrm{C}-\mathrm{C}-4\right)$, 123.08 (s, C-5), $121.34\left(\mathrm{q},{ }^{1} J_{C-F}=275.4 \mathrm{~Hz}, \mathrm{C}(2) C F_{3}\right), 113.04$ (dq, $\left.{ }^{3} J_{C-F}=9.9,{ }^{3} J_{C-F}=2.0 \mathrm{~Hz}, C-3\right), 96.75\left(d,{ }^{1} J_{C-F}=244.6 \mathrm{~Hz}\right.$, $\mathrm{C}(4) \mathrm{CHFCl}$ ) ppm. $\mathrm{C}_{11} \mathrm{H}_{6} \mathrm{~F}_{4} \mathrm{NCl}$ (263): calcd (\%) N 5.31, C 50.07, H 2.27, found $\mathrm{N}$ 5.11, C 50.19, H 2.65. HRMS (ESI +) for $\mathrm{C}_{11} \mathrm{H}_{7} \mathrm{~F}_{4} \mathrm{NCl}[\mathrm{M}+\mathrm{H}]$ : calcd 264.0198, found 264.0231. MP: 49.4 $-50.4{ }^{\circ} \mathrm{C}$.

4-(1,2,2,2-Tetrafluoroethyl)-2-(trifluoromethyl)quinoline 2aiii. The product was prepared according to the general procedure and starting from an activated solution of Ishikawa's reagent 4c (1.2 equiv., $0.95 \mathrm{~mL}, 3.26 \mathrm{mmol}$ ) by $\left(\mathrm{BF}_{3} \bullet \mathrm{Et}_{2} \mathrm{O}\right)$ (1.2 equiv., $0.41 \mathrm{~mL}, 3.26 \mathrm{mmol})$ and $\mathrm{N}$-(1,1,1-trifluoropropan-2ylidene)aniline 1a (1 equiv., $508 \mathrm{mg}, 2.71 \mathrm{mmol}$ ) in presence of anhydrous acetonitrile. 4-(1,2,2,2-Tetrafluoroethyl)-2(trifluoromethyl)quinoline 2aiii was provided after purification using a gradient of ethyl acetate in pentane (0-5\%) as a light brown solid (112 mg, 14\%, estimated). ${ }^{1} \mathrm{H} N M R \delta \mathrm{H}=8.32(1 \mathrm{H}$, 
d, $\left.{ }^{3} J_{H-H}=8.4 \mathrm{~Hz}, \mathrm{C}(8) \mathrm{H}\right), 7.99-7.97(2 \mathrm{H}, \mathrm{m}, \mathrm{C}(3,5) \mathrm{H}), 7.89(1 \mathrm{H}$, $\left.\mathrm{t}, 3 \mathrm{~J}_{\mathrm{H}-\mathrm{H}}=8.4 \mathrm{~Hz}, \mathrm{C}(7) \mathrm{H}\right), 7.78\left(1 \mathrm{H}, \mathrm{t}, 3 \mathrm{~J}_{\mathrm{H}-\mathrm{H}}=8.3 \mathrm{~Hz}, \mathrm{C}(6) \mathrm{H}\right), 6.46$ $\left(\mathrm{dq},{ }^{2} J_{\mathrm{H}-\mathrm{F}}=43.9,{ }^{3} J_{\mathrm{H}-\mathrm{F}}=5.5 \mathrm{~Hz}, \mathrm{C}\left(4-\mathrm{CHFCF}_{3}\right) \mathrm{H}\right) \mathrm{ppm} .{ }^{19} \mathrm{~F} N \mathrm{NMR} \delta \mathrm{F}$ $=-67.72\left(3 \mathrm{~F}, \mathrm{~s}, \mathrm{C} 2-\mathrm{CF}_{3}\right),-77.25\left(3 \mathrm{~F}, \mathrm{dd},{ }^{3} \mathrm{~J}_{F-F}=13.1,{ }^{3} \mathrm{~J}_{F-H}=5.8\right.$ $\left.\mathrm{Hz}, \mathrm{C} 4-\mathrm{CHFCF}_{3}\right),-198.87\left(1 \mathrm{~F}, \mathrm{dq},{ }^{2} \mathrm{~J}_{F-H}=44.3,3 J_{F-F}=12.8 \mathrm{~Hz}, \mathrm{C} 4-\right.$ ${\left.\mathrm{CH} F C F_{3}\right) . p p m .}^{13} \mathrm{C}$ NMR $\delta \mathrm{C}=147.72\left(\mathrm{q},{ }^{2} J_{C-F}=35.4 \mathrm{~Hz}, \mathrm{C}-2\right)$, 147.51 (s, C-8-C-N), $137.94\left(\mathrm{~d},{ }^{2} J_{C-F}=19.1 \mathrm{~Hz}, \mathrm{C}-4\right), 131.34$ (s, C7), 131.16 (s, C-8), 130.06 (s, C-6), 125.89 (d, C-5-C-C-4, ${ }^{2} J_{C-F}=$ $3.7 \mathrm{~Hz}$ ), $122.72(\mathrm{~s}, \mathrm{C}-5), 121.91\left(\mathrm{q},{ }^{1} \mathrm{~J}_{\mathrm{C}-F}=282.6 \mathrm{~Hz}, \mathrm{C}(4) \mathrm{CHCF}_{3}\right)$, $121.68\left(\mathrm{q},{ }^{1} J_{C-F}=282.6 \mathrm{~Hz}, \mathrm{C}(2) C F_{3}\right), 115.77\left(\mathrm{~d},{ }^{3} J_{C-F}=10.4 \mathrm{~Hz}, \mathrm{C}-\right.$ 3), $85.41\left(\mathrm{dq},{ }^{1} J_{C-F}=189.9,{ }^{2} J_{C-F}=36.1 \mathrm{~Hz}, C(4) C H F C F_{3}\right) \mathrm{ppm}$. $\mathrm{C}_{12} \mathrm{H}_{6} \mathrm{~F}_{7} \mathrm{~N}$ (297): calcd (\%) N 4.71, C 48.45, H 2.02, found N 4.71, C 48.80, H 2.32. HRMS (ESI +) for $\mathrm{C}_{11} \mathrm{H}_{7} \mathrm{~F}_{7} \mathrm{~N}[\mathrm{M}+\mathrm{H}]$ : calcd 298.0461, found 298.0462. MP: $58-58.8^{\circ} \mathrm{C}$.

\section{4-(Difluoromethyl)-8-methoxy-2-(trifluoromethyl)quinoline}

2b. The product was prepared according to the general procedure and starting from an activated solution of TFEDMA 4a (1.2 equiv., $0.33 \mathrm{~mL}, 2.82 \mathrm{mmol}$ ) by $\left(\mathrm{BF}_{3} \bullet \mathrm{Et}_{2} \mathrm{O}\right.$ ) (1.2 equiv., $0.357 \mathrm{~mL}, 2.82 \mathrm{mmol})$ and 2-methoxy- $N-(1,1,1-$ trifluoropropan-2-ylidene)aniline 1b (1 equiv., $510 \mathrm{mg}, 2.35$ mmol) in presence of anhydrous acetonitrile. 4(Difluoromethyl)-8-methoxy-2-(trifluoromethyl)quinoline $\mathbf{2 b}$ was provided after purification using a gradient of ethyl acetate in pentane (10-25\%) as a yellow solid $(575 \mathrm{mg}, 88 \%)$. ${ }^{1} \mathrm{H} N M R \delta H=7.97(1 \mathrm{H}, \mathrm{s}, \mathrm{C}(5) \mathrm{H}), 7.74-7.63(2 \mathrm{H}, \mathrm{m}, \mathrm{C}(6,7) \mathrm{H})$, $7.21\left(1 \mathrm{H}, \mathrm{d},{ }^{4} J_{H-F}=7.8 \mathrm{~Hz}, \mathrm{C}(3) \mathrm{H}\right), 7.20\left(1 \mathrm{H}, \mathrm{t},{ }^{2} J_{H-F}=54.3 \mathrm{~Hz}\right.$, $\left.\mathrm{C}\left(4-\mathrm{CHF}_{2}\right) \mathrm{H}\right), 4.12(3 \mathrm{H}, \mathrm{s}, \mathrm{Me}) \mathrm{ppm} .{ }^{19} \mathrm{~F} \mathrm{NMR} \delta \mathrm{F}=-67.16(3 \mathrm{~F}, \mathrm{~s}$, $\left.\mathrm{CF}_{3}\right),-116.17\left(2 \mathrm{~F}, \mathrm{~d}, 2 J_{F-H}=54.4 \mathrm{~Hz}, \mathrm{CHF}_{2}\right) \mathrm{ppm} .{ }^{13} \mathrm{C} \mathrm{NMR} \delta \mathrm{C}=$ $156.58(\mathrm{~s}, \mathrm{C}-8), 146.50\left(\mathrm{q},{ }^{2} J_{C-F}=35.7 \mathrm{~Hz}, \mathrm{C}-2\right), 140.11\left(\mathrm{t},{ }^{2} J_{C-F}=\right.$ $22.2 \mathrm{~Hz}, \mathrm{C}-4), 139.95$ (s, C-8-C-N), 130.74 (s, C-6), 126.43 (s, C5-C-C-4), $121.40\left(\mathrm{q},{ }^{1} J_{C-F}=275.2 \mathrm{~Hz}, \mathrm{C}(2) C F_{3}\right), 114.70-114.16$ (2 C, m, C-5, 7), 112.55 (t, $\left.{ }^{1} J_{C-F}=241.5 \mathrm{~Hz}, \mathrm{C}(4) C H F_{2}\right), 109.56$ (s, C-3), 56.58 (s, Me) ppm. $\mathrm{C}_{12} \mathrm{H}_{8} \mathrm{~F}_{5} \mathrm{NO}$ (277): calcd (\%) N 5.05, C 51.95, H 2.88, found N 5.16, C 51.92, H 2.74. MP: $72.5-76.8$ ${ }^{\circ} \mathrm{C}$.

4-(Difluoromethyl)-7-methoxy-2-(trifluoromethyl)quinoline

2c. The product was prepared according to the general procedure and starting from an activated solution of TFEDMA 4a (1.2 equiv., $0.324 \mathrm{~mL}, 2.77 \mathrm{mmol})$ by $\left(\mathrm{BF}_{3} \bullet \mathrm{Et}_{2} \mathrm{O}\right)$ (1.2 equiv., $0.35 \mathrm{~mL}, 2.77 \mathrm{mmol}$ ) and 3-methoxy- $\mathrm{N}$-(1,1,1-trifluoropropan2-ylidene)aniline 1c (1 equiv., $500 \mathrm{mg}, 2.3 \mathrm{mmol}$ ) in presence of anhydrous acetonitrile. 4-(Difluoromethyl)-7-methoxy-2(trifluoromethyl)quinoline 2c was provided after purification using a gradient of ethyl acetate in pentane (10-25\%) as a light brown solid (407 mg, 64\%). ${ }^{1} \mathrm{H} N M R \delta H=8.00\left(1 \mathrm{H}, \mathrm{d}^{3} \mathrm{~J}_{\mathrm{H}-\mathrm{H}}=\right.$ $9.3 \mathrm{~Hz}, \mathrm{C}(5) \mathrm{H}), 7.76(1 \mathrm{H}, \mathrm{s}, \mathrm{C}(3) \mathrm{H}), 7.57\left(1 \mathrm{H}, \mathrm{d},{ }^{4} J_{H-H}=2.6 \mathrm{~Hz}\right.$, $\mathrm{C}(8) \mathrm{H}), 7.39\left(1 \mathrm{H}, \mathrm{dd}, 3^{3} \mathrm{H}_{\mathrm{H}-\mathrm{H}}=9.3,{ }^{4} \mathrm{~J}_{\mathrm{H}-\mathrm{H}}=2.6 \mathrm{~Hz}, \mathrm{C}(6) \mathrm{H}\right), 7.14(1 \mathrm{H}$, $\left.\left.\mathrm{t},{ }^{2} \mathrm{~J}_{\mathrm{H}-\mathrm{F}}=54.3 \mathrm{~Hz}, \mathrm{C}\left(4-\mathrm{CHF}_{2}\right) \mathrm{H}\right)\right), 3.97(3 \mathrm{H}, \mathrm{s}, \mathrm{Me}) \mathrm{ppm} .{ }^{19} \mathrm{~F}$ NMR $\delta \mathrm{F}=-67.74\left(3 \mathrm{~F}, \mathrm{~s}, \mathrm{CF}_{3}\right),-115.04\left(2 \mathrm{~F}, \mathrm{~d},{ }^{2} \mathrm{~J}_{\mathrm{F}-\mathrm{H}}=54.4 \mathrm{~Hz}, \mathrm{C} 4-\mathrm{CHF}_{2}\right)$ ppm. ${ }^{13} \mathrm{C}$ NMR $\delta \mathrm{C}=161.92$ (s, C-7), 150.09 (s, C-8-C-N), 148.04 $\left(q,{ }^{2} J_{C-F}=35.2 \mathrm{~Hz}, \mathrm{C}-2\right), 140.01\left(\mathrm{t},{ }^{2} J_{C-F}=22.4 \mathrm{~Hz}, \mathrm{C}-4\right), 124.36(\mathrm{~s}$, $\mathrm{C}-5), 123.61$ (s, C-6), $121.26\left(\mathrm{q},{ }^{1} J_{C-F}=275.2 \mathrm{~Hz}, \mathrm{C}(2) C F_{3}\right)$, $120.32\left(\mathrm{t},{ }^{3} J_{C-F}=2.8 \mathrm{~Hz}, \mathrm{C}-5-C-C-4\right), 112.86\left(\mathrm{t},{ }^{1} J_{C-F}=241.4 \mathrm{~Hz}\right.$, $\left.\mathrm{C}(4) C H F_{2}\right), 111.81\left(\mathrm{td},{ }^{3} J_{C-F}=8.0,{ }^{3} J_{C-F}=2.3 \mathrm{~Hz}, \mathrm{C}-3\right), 108.56(\mathrm{~s}$, $\mathrm{C}-8$ ), 55.91 (s, Me) ppm. $\mathrm{C}_{12} \mathrm{H}_{8} \mathrm{~F}_{5} \mathrm{NO}$ (277): calcd (\%) N 5.05, C
51.95, H 2.88, found N 5.14, C 51.71, H 2.87. MP: $83.4-87.2$ ${ }^{\circ} \mathrm{C}$.

4-(Difluoromethyl)-6-methoxy-2-(trifluoromethyl)quinoline

2d. The product was prepared according to the general procedure and starting from an activated solution of TFEDMA 4a (1.2 equiv., $0.325 \mathrm{~mL}, 2.78 \mathrm{mmol}$ ) by $\left(\mathrm{BF}_{3} \bullet \mathrm{Et}_{2} \mathrm{O}\right)$ (1.2 equiv., $0.352 \mathrm{~mL}, 2.78 \mathrm{mmol})$ and 4-methoxy- $N$ - $(1,1,1-$ trifluoropropan-2-ylidene)aniline 1d (1 equiv., 502 mg, 2.31 $\mathrm{mmol}$ ) in presence of anhydrous acetonitrile. 4(Difluoromethyl)-6-methoxy-2-(trifluoromethyl)quinoline $\mathbf{2 d}$ was provided after purification using a gradient of ethyl acetate in pentane (10-25\%) as a brown solid (543 $\mathrm{mg}, 85 \%)$. ${ }^{1} \mathrm{H} N M R \delta \mathrm{H}=8.16\left(1 \mathrm{H}, \mathrm{d},{ }^{3} J_{\mathrm{H}-\mathrm{H}}=9.3 \mathrm{~Hz}, \mathrm{C}(8) \mathrm{H}\right), 7.85(1 \mathrm{H}, \mathrm{s}$, $\mathrm{C}(3) \mathrm{H}), 7.50\left(1 \mathrm{H}, \mathrm{dd},{ }^{3} J_{H-H}=9.3,{ }^{4} J_{H-H}=2.6 \mathrm{~Hz}, \mathrm{C}(7) \mathrm{H}\right), 7.29(1$ $\left.\mathrm{H}, \mathrm{s}, \mathrm{C}(5) \mathrm{H}), 7.11\left(1 \mathrm{H}, \mathrm{t},{ }^{2} \mathrm{~J}_{\mathrm{H}-\mathrm{F}}=54.3 \mathrm{~Hz}, \mathrm{C}\left(4-\mathrm{CHF}_{2}\right) \mathrm{H}\right)\right), 3.97(3 \mathrm{H}$, $\mathrm{s}, \mathrm{Me}) \mathrm{ppm} .{ }^{19} \mathrm{~F} N M R \delta \mathrm{F}=-67.30\left(3 \mathrm{~F}, \mathrm{~s}, \mathrm{CF}_{3}\right),-115.95(2 \mathrm{~F}, \mathrm{~d}$, $\left.{ }^{2} J_{F-H}=54.4 \mathrm{~Hz}, \mathrm{C} 4-\mathrm{CHF}_{2}\right) \mathrm{ppm} .{ }^{13} \mathrm{C} \mathrm{NMR} \delta \mathrm{C}=160.35(\mathrm{~s}, \mathrm{C}-6)$, $144.95\left(\mathrm{q},{ }^{2} \mathrm{~J}_{\mathrm{C}-\mathrm{F}}=35.4 \mathrm{~Hz}, \mathrm{C}-2\right), 144.11$ (s, C-8-C-N), 138.21 (t, $\left.{ }^{2} J_{C-F}=22.1 \mathrm{~Hz}, \mathrm{C}-4\right), 132.56(\mathrm{~s}, \mathrm{C}-8), 126.63$ (s, C-5-C-C-4), 124.40 (s, C-7), $121.43\left(\mathrm{q},{ }^{1} \mathrm{C}_{\mathrm{C}-F}=274.7 \mathrm{~Hz}, \mathrm{C}(2) C F_{3}\right), 114.59$ (td, $\left.{ }^{3} J_{C-F}=8.1,{ }^{3} J_{C-F}=2.3 \mathrm{~Hz}, \mathrm{C}-3\right), 113.17\left(\mathrm{t},{ }^{1} J_{C-F}=241.1 \mathrm{~Hz}\right.$, $\mathrm{C}(4) \mathrm{CHF}_{2}$ ), 101.06 (s, C-3), 55.88 (s, Me) ppm. $\mathrm{C}_{12} \mathrm{H}_{8} \mathrm{~F}_{5} \mathrm{NO}$ (277): calcd (\%) N 5.05, C 51.95, H 2.88, found N 5.03, C 51.64, H 2.80. MP: $105.9-108.2^{\circ} \mathrm{C}$.

4-(Difluoromethyl)-8-fluoro-2-(trifluoromethyl)quinoline 2 e. The product was prepared according to the general procedure and starting from an activated solution of TFEDMA 4a (1.2 equiv., $0.347 \mathrm{~mL}, 2.97 \mathrm{mmol})$ by $\left(\mathrm{BF}_{3} \bullet \mathrm{Et}_{2} \mathrm{O}\right)$ (1.2 equiv., 0.376 $\mathrm{mL}, 2.97 \mathrm{mmol})$ and 2-fluoro- $N$-(1,1,1- trifluoropropan-2ylidene)aniline 1e (1 equiv., $507 \mathrm{mg}, 2.47 \mathrm{mmol}$ ) in presence of anhydrous acetonitrile. 4-(Difluoromethyl)-8-fluoro-2(trifluoromethyl)quinoline $\mathbf{2 e}$ was provided after purification using a gradient of ethyl acetate in pentane $(0-5 \%)$ as an orange solid (466 mg, 70\%). ${ }^{1} \mathrm{H} \mathrm{NMR} \delta \mathrm{H}=7.99(1 \mathrm{H}, \mathrm{s}, \mathrm{C}(3) \mathrm{H})$, $7.94\left(1 \mathrm{H}, \mathrm{d},{ }^{3} J_{\mathrm{H}-\mathrm{H}}=8.6 \mathrm{~Hz}, \mathrm{C}(5) \mathrm{H}\right), 7.76\left(1 \mathrm{H}, \mathrm{td},{ }^{3} \mathrm{~J}_{\mathrm{H}-\mathrm{H}}=8.2,{ }^{4} J_{H-F}\right.$ $=5.0 \mathrm{~Hz}, \mathrm{C}(6) \mathrm{H}), 7.63-7.55(1 \mathrm{H}, \mathrm{m}, \mathrm{C}(7) \mathrm{H}), 7.20\left(1 \mathrm{H}, \mathrm{t},{ }^{2} J_{\mathrm{H}-\mathrm{F}}=\right.$ $\left.\left.54.1 \mathrm{~Hz}, \mathrm{C}\left(4-\mathrm{CHF}_{2}\right) \mathrm{H}\right)\right)$ ppm. ${ }^{19} \mathrm{~F} \mathrm{NMR} \delta \mathrm{F}=-67.64\left(3 \mathrm{~F}, \mathrm{~s}, \mathrm{CF}_{3}\right)$, $115.74\left(2 \mathrm{~F}, \mathrm{~d}, 2 J_{F-H}=54.2 \mathrm{~Hz}, \mathrm{C} 4-\mathrm{CHF}_{2}\right),-119.97\left(1 \mathrm{~F}, \mathrm{dd},{ }^{3} J_{F-H}=\right.$ $\left.10.5,{ }^{4} J_{F-H}=5.4 \mathrm{~Hz}, \mathrm{~F}\right) \mathrm{ppm} .{ }^{13} \mathrm{C} \mathrm{NMR} \delta \mathrm{C}=158.51\left(\mathrm{~d},{ }^{1} J_{C-F}=\right.$ $261.9 \mathrm{~Hz}, \mathrm{C}-8), 147.96\left(\mathrm{qd},{ }^{2} J_{C-F}=34.4 \mathrm{~Hz},{ }^{4} J_{C-F}=1.7 \mathrm{~Hz}, \mathrm{C}-2\right)$, $140.33\left(\mathrm{td},{ }^{2} J_{C-F}=22.6,{ }^{4} J_{C-F}=2.9 \mathrm{~Hz}, \mathrm{C}-4\right), 138.40\left(\mathrm{~d},{ }^{2} J_{C-F}=12.2\right.$ $\mathrm{Hz}, \mathrm{C}-8-\mathrm{C}-\mathrm{N}$ ), 130.35 (d, $\left.{ }^{3} J_{C-F}=8.1 \mathrm{~Hz}, \mathrm{C}-6\right), 126.50$ (s, C-5-C-C4), $120.95\left(\mathrm{q},{ }^{1} J_{C-F}=275.5 \mathrm{~Hz}, \mathrm{C}(2) C F_{3}\right), 119.25\left(\mathrm{~d},{ }^{4} J_{C-F}=5.3 \mathrm{~Hz}\right.$, C-5), $115.90\left(\mathrm{~d},{ }^{2} J_{C-F}=18.6 \mathrm{~Hz}, \mathrm{C}-7\right), 115.20\left(\mathrm{t},{ }^{3} J_{C-F}=7.5 \mathrm{~Hz}, \mathrm{C}-\right.$ 3), $112.48\left(\mathrm{t},{ }^{1} J_{C-F}=241.9 \mathrm{~Hz}, \mathrm{C}(4) \mathrm{CHF}_{2}\right) \mathrm{ppm} . \mathrm{C}_{11} \mathrm{H}_{5} \mathrm{~F}_{6} \mathrm{~N}(265)$ : calcd (\%) N 5.28, C 49.78, H 1.88, found N 5.44, C 50.00, H 1.95. MP: $49.7-51.3^{\circ} \mathrm{C}$.

4-(Difluoromethyl)-7-fluoro-2-(trifluoromethyl)quinoline $2 f$. The product was prepared according to the general procedure and starting from an activated solution of TFEDMA 4a (1.2 equiv., $0.349 \mathrm{~mL}, 2.98 \mathrm{mmol}$ ) by $\left(\mathrm{BF}_{3} \bullet \mathrm{Et}_{2} \mathrm{O}\right)$ (1.2 equiv., 0.378 $\mathrm{mL}, 2.98 \mathrm{mmol})$ and 3-fluoro- $N$-(1,1,1-trifluoropropan-2ylidene)aniline $1 \mathrm{f}$ (1 equiv., $509 \mathrm{mg}, 2.48 \mathrm{mmol}$ ) in presence of anhydrous acetonitrile. 4-(Difluoromethyl)-7-fluoro-2(trifluoromethyl)quinoline $\mathbf{2} \mathbf{f}$ was provided after purification using a gradient of ethyl acetate in pentane (0-5\%) as a yellow solid $(405 \mathrm{mg}, 62 \%){ }^{1} \mathrm{H}$ NMR $\delta=8.19\left(1 \mathrm{H}, \mathrm{dd},{ }^{4} J_{\mathrm{H}-\mathrm{H}}=9.2,{ }^{3} J_{\mathrm{H}-\mathrm{F}}=\right.$ 
$5.7 \mathrm{~Hz}, \mathrm{C}(8) \mathrm{H}), 7.93\left(1 \mathrm{H}, \mathrm{dd},{ }^{3} J_{\mathrm{H}-\mathrm{H}}=9.3,{ }^{4} J_{\mathrm{H}-\mathrm{F}}=2.3 \mathrm{~Hz}, \mathrm{C}(5) \mathrm{H}\right)$, $7.88(1 \mathrm{H}, \mathrm{s}, \mathrm{C}(3) \mathrm{H}), 7.61-7.54(1 \mathrm{H}, \mathrm{m}, \mathrm{C}(6) \mathrm{H}), 7.17\left(1 \mathrm{H},{ }^{2} \mathrm{~J}_{\mathrm{H}-\mathrm{F}}=\right.$ $\left.\left.54.1 \mathrm{~Hz}, \mathrm{C}\left(4-\mathrm{CHF}_{2}\right) \mathrm{H}\right)\right)$ ppm. ${ }^{19} \mathrm{~F} N \mathrm{NMR} \delta \mathrm{F}=-67.89\left(3 \mathrm{~F}, \mathrm{~s}, \mathrm{CF}_{3}\right)$, $105.57--105.66(1 \mathrm{~F}, \mathrm{~m}, \mathrm{~F}),-114.89\left(2 \mathrm{~F}, \mathrm{~d},{ }^{2} J_{F-H}=54.0 \mathrm{~Hz}, \mathrm{C} 4-\right.$ $\left.\mathrm{CHF}_{2}\right) \mathrm{ppm} .{ }^{13} \mathrm{C}$ NMR $\delta \mathrm{C}=163.84\left(\mathrm{~d},{ }^{1} J_{C-F}=254.8 \mathrm{~Hz}, \mathrm{C}-7\right)$, $149.36\left(\mathrm{~d},{ }^{3} J_{C-F}=13.1 \mathrm{~Hz}, \mathrm{C} 8-C-\mathrm{N}\right), 149.09\left(\mathrm{q},{ }^{2} J_{C-F}=35.6 \mathrm{~Hz}, \mathrm{C}-\right.$ 2), $140.60\left(\mathrm{td},{ }^{2} J_{C-F}=22.5,5 J_{C-F}=1.4 \mathrm{~Hz}, \mathrm{C}-4\right), 125.92\left(\mathrm{~d},{ }^{3} J_{C-F}=\right.$ $9.8 \mathrm{~Hz}, \mathrm{C}-5), 122.08(\mathrm{~s}, \mathrm{C}-5-C-\mathrm{C}-4), 121.04\left(\mathrm{q},{ }^{1} J_{C-F}=275.4 \mathrm{~Hz}\right.$, $\left.\mathrm{C}(2) C F_{3}\right), 120.77\left(\mathrm{~d},{ }^{2} J_{C-F}=25.6 \mathrm{~Hz}, \mathrm{C}-6\right), 114.89\left(\mathrm{~d},{ }^{2} J_{C-F}=20.7\right.$ $\mathrm{Hz}, \mathrm{C}-8), 113.92-113.16(\mathrm{~m}, \mathrm{C}-3), 112.81$ (t, ${ }^{1} J_{C-F}=241.8 \mathrm{~Hz}$, $\mathrm{C}(4) \mathrm{CHF}_{2}$ ) ppm. $\mathrm{C}_{11} \mathrm{H}_{5} \mathrm{~F}_{6} \mathrm{~N}$ (265): calcd (\%) $\mathrm{N} \mathrm{5.28,} \mathrm{C} \mathrm{49.78,} \mathrm{H}$ 1.88 , found N 5.36, C 49.88, H 1.77. MP: $61.6-62.8^{\circ} \mathrm{C}$.

4-(Difluoromethyl)-6-fluoro-2-(trifluoromethyl)quinoline 2gi. The product was prepared according to the general procedure and starting from an activated solution of TFEDMA 4a (1.2 equiv., $0.346 \mathrm{~mL}, 2.96 \mathrm{mmol}$ ) by $\left(\mathrm{BF}_{3} \bullet \mathrm{Et}_{2} \mathrm{O}\right)$ (1.2 equiv., 0.374 $\mathrm{mL}, 2.96 \mathrm{mmol})$ and 4-fluoro- $\mathrm{N}$-(1,1,1-trifluoropropan-2ylidene)aniline $1 \mathrm{~g}$ (1 equiv., $505 \mathrm{mg}, 2.46 \mathrm{mmol}$ ) in presence of anhydrous acetonitrile. 4-(Difluoromethyl)-6-fluoro-2(trifluoromethyl)quinoline 2gi was provided after purification using a gradient of ethyl acetate in pentane (0-5\%) as a yellow solid (434 mg, 67\%). ${ }^{1} \mathrm{H} N M R \delta H=8.32\left(1 \mathrm{H}, \mathrm{dd},{ }^{3} \mathrm{~J}_{\mathrm{H}-\mathrm{H}}=9.3,{ }^{4} J_{\mathrm{H}-\mathrm{F}}\right.$ $=5.5 \mathrm{~Hz}, \mathrm{C}(8) \mathrm{H}), 7.93(1 \mathrm{H}, \mathrm{s}, \mathrm{C}(3) \mathrm{H}), 7.81-7.73(1 \mathrm{H}, \mathrm{m}$, $\mathrm{C}(5) \mathrm{H}), 7.67-7.66(1 \mathrm{H}, \mathrm{m}, \mathrm{C}(7) \mathrm{H}), 7.12\left(1 \mathrm{H}, \mathrm{t},{ }^{2} \mathrm{~J}_{\mathrm{H}-\mathrm{F}}=54.1 \mathrm{~Hz}\right.$, $\left.\left.\mathrm{C}\left(4-\mathrm{CHF}_{2}\right) \mathrm{H}\right)\right)$ ppm. ${ }^{19} \mathrm{~F} N M R \delta \mathrm{F}=-67.69\left(3 \mathrm{~F}, \mathrm{~s}, \mathrm{CF}_{3}\right),-105.84--$ $105.77(1 \mathrm{~F}, \mathrm{~m}, \mathrm{~F}),-115.61\left(2 \mathrm{~F}, \mathrm{~d},{ }^{2} J_{F-H}=54.1 \mathrm{~Hz}, \mathrm{C} 4-\mathrm{CHF}_{2}\right.$ ) ppm. ${ }^{13} \mathrm{C}$ NMR $\delta C=162.40\left(\mathrm{~d},{ }^{1} J_{C-F}=254.3 \mathrm{~Hz}, \mathrm{C}-6\right), 147.31$ (qd, $\left.{ }^{2} J_{C-F}=35.7,{ }^{6} J_{C-F}=3.2 \mathrm{~Hz}, \mathrm{C}-2\right), 145.05(\mathrm{~s}, \mathrm{C}-8-\mathrm{C}-\mathrm{N}), 139.92(\mathrm{td}$, $\left.{ }^{2} J_{C-F}=22.5,{ }^{4} J_{C-F}=6.2 \mathrm{~Hz}, C-4\right), 133.96\left(d, 3 J_{C-F}=9.8 \mathrm{~Hz}, \mathrm{C}-8\right)$, $126.06\left(\mathrm{~d},{ }^{3} J_{C-F}=10.7 \mathrm{~Hz}, \mathrm{C}-5-C-C-4\right), 122.01\left(\mathrm{~d},{ }^{2} J_{C-F}=26.0 \mathrm{~Hz}\right.$, $\mathrm{C}-7), 121.24\left(\mathrm{q},{ }^{1} J_{C-F}=275.1 \mathrm{~Hz}, \mathrm{C}(2) C F_{3}\right), 115.17-115.02(\mathrm{~m}$, $\mathrm{C}-3), 112.74\left(\mathrm{t},{ }^{1} J_{C-F}=237.4 \mathrm{~Hz}, \mathrm{C}(4) C H F_{2}\right), 107.68\left(\mathrm{~d},{ }^{2} J_{C-F}=24.1\right.$ $\mathrm{Hz}, \mathrm{C}-5)$ ppm. HRMS (ESI +) for $\mathrm{C}_{11} \mathrm{H}_{6} \mathrm{~F}_{6} \mathrm{~N}[\mathrm{M}+\mathrm{H}]$ : calcd 266.0399, found 266.0387. MP: $68.2-69.8^{\circ} \mathrm{C}$.

\section{4-[Chloro(fluoro)methyl]-6-fluoro-2-}

(trifluoromethyl)quinoline 2gii. The product was prepared according to the general procedure and starting from an activated solution of Yarovenko's reagent 4b (1.2 equiv., 0.675 $\mathrm{mL}, 2.97 \mathrm{mmol})$ by $\left(\mathrm{BF}_{3} \bullet \mathrm{Et}_{2} \mathrm{O}\right)(1.2$ equiv., $0.376 \mathrm{~mL}, 2.97$ $\mathrm{mmol}$ ) and 4-fluoro- $N$-(1,1,1-trifluoropropan-2-ylidene)aniline $1 \mathrm{~g}$ (1 equiv., $507 \mathrm{mg}, 2.47 \mathrm{mmol}$ ) in presence of anhydrous acetonitrile.

4-[Chloro(fluoro)methyl]-6-fluoro-2(trifluoromethyl)quinoline 2gii was provided after purification using a gradient of ethyl acetate in pentane $(0-5 \%)$ in mixture with the non-cyclised compound, the 1-chloro-1,5,5,5tetrafluoro-4-((4-fluorophenyl)amino)pent-3-en-2-one (6'g) with a ratio of $7: 1$ (35\% by $\left.{ }^{19} \mathrm{~F} N M R\right) .{ }^{1} \mathrm{H} \delta \mathrm{H}=8.32\left(1 \mathrm{H}, \mathrm{dd},{ }^{3} \mathrm{~J}_{\mathrm{H}}\right.$ $\left.H=9.3,{ }^{4} J_{H-F}=5.5 \mathrm{~Hz}, \mathrm{C}(8) \mathrm{H}\right), 7.94(1 \mathrm{H}, \mathrm{s}, \mathrm{C}(3) \mathrm{H}), 7.75(1 \mathrm{H}, \mathrm{dd}$, $\left.{ }^{3} J_{H-F}=9.4,{ }^{4} J_{H-H}=2.7 \mathrm{~Hz}, C(5) \mathrm{H}\right), 7.65\left(1 \mathrm{H}, \mathrm{ddd},{ }^{3} J_{H-F}=9.4,{ }^{4} J_{H-F}\right.$ $\left.=7.9,{ }^{4} J_{H-H}=2.7 \mathrm{~Hz}, \mathrm{C}(7) \mathrm{H}\right), 7.54\left(1 \mathrm{H}, \mathrm{d},{ }^{2} J_{H-F}=48.7 \mathrm{~Hz}, \mathrm{C}(4-\right.$ (HFCl)H) ppm.

\section{6-Fluoro-4-(1,2,2,2-tetrafluoroethyl)-2-}

(trifluoromethyl)quinoline 2giii. The product was prepared according to the general procedure and starting from an activated solution of Ishikawa's reagent 4c (1.2 equiv., 0.854 $\mathrm{mL}, 2.92 \mathrm{mmol})$ by $\left(\mathrm{BF}_{3} \bullet \mathrm{Et}_{2} \mathrm{O}\right)$ (1.21 equiv., $0.372 \mathrm{~mL}, 2.93$ $\mathrm{mmol}$ ) and 4-fluoro- $N$-(1,1,1-trifluoropropan-2-ylidene)aniline
$1 \mathrm{~g}$ (1 equiv., $498 \mathrm{mg}, 2.43 \mathrm{mmol}$ ) in presence of anhydrous acetonitrile. 6-Fluoro-4-(1,2,2,2-tetrafluoroethyl)-2(trifluoromethyl)quinoline 2giii was provided after purification using a gradient of ethyl acetate in pentane (0-5\%) in mixture with the non-cyclized compound, the $1,1,1,2,6,6,6$ heptafluoro-5-((4-fluorophenyl)amino)hex-4-en-3-one (6'giii) with a ratio of 1:3. as a brown oil $(64.7 \mathrm{mg}) .{ }^{1} \mathrm{H} \mathrm{NMR} \delta \mathrm{H}=8.35$ $\left(1 \mathrm{H}, \mathrm{dd},{ }^{3} J_{\mathrm{H}-\mathrm{H}}=9.3,{ }^{4} \mathrm{~J}_{\mathrm{H}-\mathrm{F}}=5.5 \mathrm{~Hz}, \mathrm{C}(8) \mathrm{H}\right), 7.97(1 \mathrm{H}, \mathrm{s}, \mathrm{C}(3) \mathrm{H})$, $7.72-7.65(1 \mathrm{H}, \mathrm{m}, \mathrm{C}(5) \mathrm{H}), 7.64-7.62(1 \mathrm{H}, \mathrm{m}, \mathrm{C}(7) \mathrm{H}), 6.33(1$ $\left.\mathrm{H}, \mathrm{dq},{ }^{2} J_{\mathrm{H}-\mathrm{F}}=43.8,{ }^{3} J_{\mathrm{H}-\mathrm{F}}=5.4 \mathrm{~Hz}, \mathrm{C}\left(4-\mathrm{CHFCF}_{3}\right) \mathrm{H}\right)$ ppm. HRMS (ESI + ) for $\mathrm{C}_{12} \mathrm{H}_{6} \mathrm{~F}_{8} \mathrm{~N}[\mathrm{M}+\mathrm{H}]$ : calcd 316.0367, found 316.0375 .

\section{4-(Difluoromethyl)-7-(trifluoromethoxy)-2-}

(trifluoromethyl)quinoline $\mathbf{2 h}$. The product was prepared according to the general procedure and starting from an activated solution of TFEDMA 4a (1.2 equiv., $0.259 \mathrm{~mL}, 2.21$ $\mathrm{mmol}$ ) by $\left(\mathrm{BF}_{3} \bullet \mathrm{Et}_{2} \mathrm{O}\right)$ (1.2 equiv., $0.28 \mathrm{~mL}, 2.21 \mathrm{mmol}$ ) and 3(trifluoromethoxy)- $\mathrm{N}$-(1,1,1-trifluoropropan-2-ylidene)aniline 1h (1 equiv., $500 \mathrm{mg}, 1.84 \mathrm{mmol}$ ) in presence of anhydrous acetonitrile. 4-(Difluoromethyl)-7-(trifluoromethoxy)-2(trifluoromethyl)quinoline $\mathbf{2} \mathbf{h}$ was provided after purification using a gradient of ethyl acetate in pentane (0-5\%) as a yellow solid $(219 \mathrm{mg}, 36 \%) .{ }^{1} \mathrm{H}$ NMR $\delta \mathrm{H}=8.24\left(1 \mathrm{H}, \mathrm{d},{ }^{3} \mathrm{~J}_{\mathrm{H}-\mathrm{H}}=9.3 \mathrm{~Hz}\right.$, $\mathrm{C}(5) \mathrm{H}), 8.16(1 \mathrm{H}, \mathrm{s}, \mathrm{C}(8) \mathrm{H}), 7.94(1 \mathrm{H}, \mathrm{s}, \mathrm{C}(3) \mathrm{H}), 7.65(1 \mathrm{H}, \mathrm{dd}$, $\left.{ }^{3} J_{H-H}=9.3,4 J_{H-H}=2.4 \mathrm{~Hz}, \mathrm{C}(6) \mathrm{H}\right), 7.18\left(1 \mathrm{H}, \mathrm{t},{ }^{2} J_{H-F}=54.1 \mathrm{~Hz}\right.$, $\left.\mathrm{C}\left(4-\mathrm{CHF}_{2}\right) \mathrm{H}\right) \mathrm{ppm} .{ }^{19} \mathrm{~F} \mathrm{NMR} \delta \mathrm{F}=-57.88\left(3 \mathrm{~F}, \mathrm{~s}, \mathrm{OCF}_{3}\right),-67.89(3$ $\left.\mathrm{F}, \mathrm{s}, \mathrm{CF}_{3}\right),-114.87\left(2 \mathrm{~F}, \mathrm{~d},{ }^{2} \mathrm{~J}_{F-H}=54.1 \mathrm{~Hz}, \mathrm{C} 4-\mathrm{CHF}_{2}\right)$ ppm. ${ }^{13} \mathrm{C}$ NMR $\delta C=151(\mathrm{~s}, \mathrm{C}-7), 149.37\left(\mathrm{q},{ }^{2} \mathrm{~J}_{\mathrm{C}-F}=35.8 \mathrm{~Hz}, \mathrm{C}-2\right), 148.63$ (s, C-8-C-N), $140.65\left(\mathrm{t},{ }^{2} J_{C-F}=22.6 \mathrm{~Hz}, \mathrm{C}-4\right), 125.80(\mathrm{~s}, \mathrm{C}-5)$, 123.99 (s, C-6), $123.23-123.19$ (m, C-5-C-C-4), 121.07 (q, ${ }^{1} J_{C-F}$ $\left.=276.7 \mathrm{~Hz}, \mathrm{C}(2) C F_{3}\right), 120.55\left(\mathrm{q},{ }^{1} J_{C-F}=260.0 \mathrm{~Hz}, \mathrm{OCF}_{3}\right), 120.25$ $(\mathrm{s}, \mathrm{C}-8), 114.60\left(\mathrm{td},{ }^{3} J_{C-F}=8.0,{ }^{3} J_{C-F}=2.0 \mathrm{~Hz}, \mathrm{C}-3\right), 112.72\left(\mathrm{t},{ }^{1} J_{C-F}\right.$ $=242.0 \mathrm{~Hz}, \mathrm{C}(4) \mathrm{CHF}_{2}$ ) ppm. $\mathrm{C}_{12} \mathrm{H}_{5} \mathrm{~F}_{8} \mathrm{NO}$ (331): calcd (\%) $\mathrm{N} 4.23$, C 43.48, H 1.51, found N 4.28, C 43.94, H 1.35. MP: $36.3-37.8$ ${ }^{\circ} \mathrm{C}$.

\section{4-(Difluoromethyl)-6-(trifluoromethoxy)-2-}

(trifluoromethyl)quinoline $\mathbf{2 i}$. The product was prepared according to the general procedure and starting from an activated solution of TFEDMA 4a (1.2 equiv., $0.278 \mathrm{~mL}, 2.38$ $\mathrm{mmol}$ ) by $\left(\mathrm{BF}_{3} \bullet \mathrm{Et}_{2} \mathrm{O}\right)$ (1.2 equiv., $0.282 \mathrm{~mL}, 2.22 \mathrm{mmol}$ ) and of 4-(trifluoromethoxy)- $N$-(1,1,1-trifluoropropan-2-ylidene)aniline 1i (1 equiv., $584 \mathrm{mg}, 1.85 \mathrm{mmol}$ ) in presence of anhydrous acetonitrile. 4-(Difluoromethyl)-6-(trifluoromethoxy)-2(trifluoromethyl)quinoline $\mathbf{2} \mathbf{i}$ was provided after purification using a gradient of ethyl acetate in pentane (0-5\%) as a yellow solid (300.3 mg, 49\%). ${ }^{1} \mathrm{H} N M R \delta H=8.39\left(1 \mathrm{H}, \mathrm{d},{ }^{3} J_{H-H}=9.3 \mathrm{~Hz}\right.$, $\mathrm{C}(8) \mathrm{H}), 7.97(2 \mathrm{H}, \mathrm{s}, \mathrm{C}(3,5) \mathrm{H}), 7.77\left(1 \mathrm{H}, \mathrm{dd},{ }^{3} J_{H-H}=9.3,{ }^{4} J_{H-H}=\right.$ $1.9 \mathrm{~Hz}, \mathrm{C}(7) \mathrm{H}), 7.15\left(1 \mathrm{H}, \mathrm{t},{ }^{2} \mathrm{~J}_{\mathrm{H}-\mathrm{F}}=54.0 \mathrm{~Hz}, \mathrm{C}\left(4-\mathrm{CHF}_{2}\right) \mathrm{H}\right) \mathrm{ppm} .{ }^{19} \mathrm{~F}$ $\mathrm{NMR} \delta \mathrm{F}=-57.72\left(3 \mathrm{~F}, \mathrm{~s}, \mathrm{OCF}_{3}\right),-67.79\left(3 \mathrm{~F}, \mathrm{~s}, \mathrm{CF}_{3}\right),-115.31(2 \mathrm{~F}$, d, $\left.{ }^{2} J_{F-H}=53.9 \mathrm{~Hz}, \mathrm{C} 4-\mathrm{CHF}_{2}\right)$ ppm. ${ }^{13} \mathrm{C} \mathrm{NMR} \delta \mathrm{C}=149.50(\mathrm{~s}, \mathrm{C}-6)$, $148.41\left(\mathrm{q},{ }^{2} J_{C-F}=35.9 \mathrm{~Hz}, \mathrm{C}-2\right), 146.02(\mathrm{~s}, \mathrm{C}-8-\mathrm{C}-\mathrm{N}), 140.46$ (t, $\left.2 J_{C-F}=22.6 \mathrm{~Hz}, \mathrm{C}-4\right), 133.66(\mathrm{~s}, \mathrm{C}-8), 125.52$ (s, C-5-C-C-4), 125.35 (s, C-7), $121.12\left(\mathrm{q},{ }^{1} J_{C-F}=276.4 \mathrm{~Hz}, \mathrm{C}(2) C F_{3}\right), 120.74$ (q, $\left.{ }^{1} J_{C-F}=260.6 \mathrm{~Hz}, \mathrm{OCF}_{3}\right), 115.36\left(\mathrm{td},{ }^{3} J_{C-F}=8.0,{ }^{3} J_{C-F}=2.1 \mathrm{~Hz}, \mathrm{C}-3\right)$, 113.97 (s, C-5), $112.67\left(\mathrm{t},{ }^{1} \mathrm{~J}_{\mathrm{C}-\mathrm{F}}=242.0 \mathrm{~Hz}, \mathrm{C}(4) \mathrm{CHF}_{2}\right) \mathrm{ppm}$. $\mathrm{C}_{12} \mathrm{H}_{5} \mathrm{~F}_{8} \mathrm{NO}$ (331): calcd (\%) N 4.23, C 43.48, H 1.51, found $\mathrm{N}$ 4.20, C 43.77, H 1.83. MP: $42.2-43.8^{\circ} \mathrm{C}$. 
4-(Difluoromethyl)-N,N-dimethyl-2-(trifluoromethyl)quinolin6-amine $\mathbf{2 j}$. The product was prepared according to the general procedure and starting from an activated solution of TFEDMA 4a (1.2 equiv., $0.305 \mathrm{~mL}, 2.61 \mathrm{mmol})$ by $\left(\mathrm{BF}_{3} \bullet \mathrm{Et}_{2} \mathrm{O}\right)$ (1.2 equiv., $0.33 \mathrm{~mL}, 2.61 \mathrm{mmol}$ ) and of $1-\mathrm{N}, \mathrm{N}$-dimethyl-4- $\mathrm{N}$ (1,1,1-trifluoropropan-2-ylidene)benzene-1,4-diamine $\quad \mathbf{1 j}$ (1 equiv., $500 \mathrm{mg}, 2.17 \mathrm{mmol}$ ) in presence of anhydrous acetonitrile. 4-(Difluoromethyl)- $N, N$-dimethyl-2(trifluoromethyl)quinolin-6-amine $\mathbf{2 j}$ was provided after purification using a gradient of ethyl acetate in pentane (0-5\%) as a yellow solid $(235 \mathrm{mg}, 37 \%)$. ${ }^{1} \mathrm{H} \mathrm{NMR} \delta \mathrm{H}=8.07\left(1 \mathrm{H}, \mathrm{d},{ }^{3} \mathrm{~J}_{\mathrm{H}-\mathrm{H}}\right.$ $=9.5 \mathrm{~Hz}, \mathrm{C}(8) \mathrm{H}), 7.75(1 \mathrm{H}, \mathrm{s}, \mathrm{C}(3) \mathrm{H}), 7.43\left(1 \mathrm{H}, \mathrm{dd}, 3_{\mathrm{H}-\mathrm{H}}=9.5\right.$, $\left.{ }^{4} J_{H-H}=2.8 \mathrm{~Hz}, \mathrm{C}(7) \mathrm{H}\right), 7.07\left(1 \mathrm{H}, \mathrm{t}^{2} \mathrm{~J}_{\mathrm{H}-\mathrm{F}}=54.5 \mathrm{~Hz}, \mathrm{C}\left(4-\mathrm{CHF}_{2}\right) \mathrm{H}\right)$, $6.84(1 \mathrm{H}, \mathrm{s}, \mathrm{C}(5) \mathrm{H}), 3.14\left(1 \mathrm{H}, \mathrm{s}, \mathrm{NMe}_{2}\right) \mathrm{ppm} .{ }^{19} \mathrm{~F} \mathrm{NMR} \delta \mathrm{F}=-$ $66.90\left(3 \mathrm{~F}, \mathrm{~s}, \mathrm{CF}_{3}\right),-117.13\left(2 \mathrm{~F}, \mathrm{~d},{ }^{2} \mathrm{~J}_{F-H}=54.7 \mathrm{~Hz}, \mathrm{C} 4-\mathrm{CHF}_{2}\right)$ ppm. ${ }^{13} \mathrm{C}$ NMR $\delta \mathrm{C}=150.28$ (s, C-6), $143.01-141.69$ (2 C, m, C2/C-8-C-N), $136.12\left(\mathrm{t},{ }^{2} J_{C-F}=21.6 \mathrm{~Hz}, \mathrm{C}-4\right), 131.80(\mathrm{~s}, \mathrm{C}-8)$, $127.25\left(\mathrm{t},{ }^{3} J_{C-F}=2.5 \mathrm{~Hz}, \mathrm{C}-5-C-\mathrm{C}-4\right), 121.98\left(\mathrm{q},{ }^{1} J_{C-F}=274.0 \mathrm{~Hz}\right.$, $\left.\mathrm{C}(2) C F_{3}\right), 120.46(\mathrm{~s}, \mathrm{C}-7), 114.48\left(\mathrm{td},{ }^{3} J_{C-F}=8.2,{ }^{3} J_{C-F}=2.3 \mathrm{~Hz}, \mathrm{C}-\right.$ 3), $113.29\left(\mathrm{t},{ }^{1} \mathrm{~J}_{\mathrm{C}-\mathrm{F}}=240.4 \mathrm{~Hz}, \mathrm{C}(4) \mathrm{CHF}_{2}\right), 98.92(\mathrm{~s}, \mathrm{C}-5), 40.46$ (2 C, s, $\mathrm{NMe}_{2}$ ) ppm. $\mathrm{C}_{13} \mathrm{H}_{11} \mathrm{~F}_{5} \mathrm{~N}_{2}$ (290): calcd (\%) N 9.65, C 53.75, H 3.79, found N 9.42, C 53.55, H 3.81. MP: $107.5-108.4{ }^{\circ} \mathrm{C}$.

4-(Difluoromethyl)-7-fluoro-8-methyl-2-

(trifluoromethyl)quinoline $\mathbf{2 k}$. The product was prepared according to the general procedure and starting from an activated solution of TFEDMA 4a (1.2 equiv., $0.644 \mathrm{~mL}, 5.5$ $\mathrm{mmol}$ ) by $\left(\mathrm{BF}_{3} \bullet \mathrm{Et}_{2} \mathrm{O}\right)$ (1.2 equiv., $0.698 \mathrm{~mL}, 5.5 \mathrm{mmol}$ ) and $3-$ fluoro-2-methyl- $N$-(1,1,1-trifluoropropan-2-ylidene)aniline $\mathbf{1 k}$ (1 equiv., $1.76 \mathrm{~g}, 4.59 \mathrm{mmol}$ ) in presence of anhydrous acetonitrile. 4-(Difluoromethyl)-7-fluoro-8-methyl-2(trifluoromethyl)quinoline $\mathbf{2 k}$ was provided after purification using a gradient of ethyl acetate in pentane (0-5\%) as a yellow solid (488.4 mg, 38\%). ${ }^{1} \mathrm{H} \mathrm{NMR} \delta \mathrm{H}=7.99\left(1 \mathrm{H}, \mathrm{dd},{ }^{3} \mathrm{~J}_{\mathrm{H}-\mathrm{H}}=9.0\right.$, $\left.{ }^{4} J_{H-F}=5.9 \mathrm{~Hz}, \mathrm{C}(5) \mathrm{H}\right), 7.88(1 \mathrm{H}, \mathrm{s}, \mathrm{C}(3) \mathrm{H}), 7.54\left(1 \mathrm{H}, \mathrm{t},{ }^{3} \mathrm{~J}_{\mathrm{H}-\mathrm{H}}=9.0\right.$ $\mathrm{Hz}, \mathrm{C}(6) \mathrm{H}), 7.17\left(1 \mathrm{H}, \mathrm{t},{ }^{2} J_{\mathrm{H}-\mathrm{F}}=54.2 \mathrm{~Hz}, \mathrm{C}\left(4-\mathrm{CHF}_{2}\right) \mathrm{H}\right), 2.75(3 \mathrm{H}$, $\left.\mathrm{d},{ }^{4} J_{H-F}=2.6 \mathrm{~Hz}, \mathrm{Me}\right) \mathrm{ppm} .{ }^{19} \mathrm{~F} N M R \delta \mathrm{F}=-67.78\left(3 \mathrm{~F}, \mathrm{~s}, \mathrm{CF}_{3}\right)$, $108.31--108.46(1 \mathrm{~F}, \mathrm{~m}, \mathrm{~F}),-114.94\left(2 \mathrm{~F}, \mathrm{~d},{ }^{2} J_{F-H}=54.2 \mathrm{~Hz}, \mathrm{C} 4-\right.$ $\mathrm{CHF}_{2}$ ) ppm. ${ }^{13} \mathrm{C}$ NMR $\delta \mathrm{C}=161.60\left(\mathrm{~d},{ }^{1} J_{C-F}=250.2 \mathrm{~Hz}, \mathrm{C}-7\right)$, $148.47-147.14$ (2 C, m, C-2/C-8-C-N), 140.54 (td, ${ }^{2} J_{C-F}=22.3$, $\left.{ }^{5} J_{C-F}=1.8 \mathrm{~Hz}, \mathrm{C}-4\right), 123.79\left(\mathrm{~d},{ }^{2} J_{C-F}=16.2 \mathrm{~Hz}, \mathrm{C}-8\right), 122.26(2 \mathrm{C}$, d, $\left.{ }^{3} J_{C-F}=10.3 \mathrm{~Hz}, C-5 / C-5-C-C-4\right), 121.32\left(q,{ }^{1} J_{C-F}=275.3 \mathrm{~Hz}\right.$, $\left.\mathrm{C}(2) C F_{3}\right), 120.25\left(\mathrm{~d},{ }^{2} J_{C-F}=27.2 \mathrm{~Hz}, \mathrm{C}-6\right), 113.49-112.94(\mathrm{~m}, \mathrm{C}-$ 3), $112.84\left(\mathrm{t},{ }^{1} J_{C-F}=241.6 \mathrm{~Hz}, \mathrm{C}(4) C H F_{2}\right), 9.38\left(\mathrm{~d},{ }^{3} J_{C-F}=3.7 \mathrm{~Hz}\right.$, $\mathrm{Me})$ ppm. HRMS $\left(\mathrm{ESI}+\right.$ ) for $\mathrm{C}_{12} \mathrm{H}_{8} \mathrm{~F}_{6} \mathrm{~N}[\mathrm{M}+\mathrm{H}]$ : calcd 280.0555, found 280.0570. MP: $78.7-79.4{ }^{\circ} \mathrm{C}$.

7-Chloro-4-(difluoromethyl)-8-methyl-2-

(trifluoromethyl)quinoline 2l. The product was prepared according to the general procedure and starting from an activated solution of TFEDMA 4 a (1.2 equiv., $0.599 \mathrm{~mL}, 5.12$ $\mathrm{mmol})$ by $\left(\mathrm{BF}_{3} \bullet \mathrm{Et}_{2} \mathrm{O}\right)$ (1.2 equiv., $0.648 \mathrm{~mL}, 5.12 \mathrm{mmol}$ ) and $3-$ chloro-2-methyl- $N$-(1,1,1-trifluoropropan-2-ylidene)aniline $\mathbf{1 l}$ (1 equiv., $2.01 \mathrm{~g}, 4.26 \mathrm{mmol}$ ) in presence of anhydrous acetonitrile. 7-Chloro-4-(difluoromethyl)-8-methyl-2(trifluoromethyl)quinoline $2 \mathbf{l}$ was provided after purification using a gradient of ethyl acetate in pentane $(0-5 \%)$ as a beige amorphous solid (485 mg, 38\%). ${ }^{1} \mathrm{H} \mathrm{NMR} \delta \mathrm{H}=7.93-7.91(2 \mathrm{H}$, $\mathrm{m}, \mathrm{C}(3,5) \mathrm{H}), 7.74\left(1 \mathrm{H}, \mathrm{d},{ }^{3} J_{H-H}=9.1 \mathrm{~Hz}, \mathrm{C}(6) \mathrm{H}\right), 7.17\left(1 \mathrm{H}, \mathrm{t},{ }^{2} J_{H-F}\right.$ $\left.=54.2 \mathrm{~Hz}, \mathrm{C}\left(4-\mathrm{CHF}_{2}\right) \mathrm{H}\right), 2.93(3 \mathrm{H}, \mathrm{s}, \mathrm{Me}) \mathrm{ppm} .{ }^{19} \mathrm{~F} \delta \mathrm{F}=-67.78(3$ $\left.\mathrm{F}, \mathrm{s}, \mathrm{CF}_{3}\right),-115.12\left(2 \mathrm{~F}, \mathrm{~d},{ }^{2} \mathrm{~J}_{F-H}=54.1 \mathrm{~Hz}, \mathrm{C} 4-\mathrm{CHF}_{2}\right) \mathrm{ppm} .{ }^{13} \mathrm{C}$ NMR $\delta C=147.53\left(q,{ }^{2} J_{C-F}=35.8 \mathrm{~Hz}, \mathrm{C}-2\right), 147.44(\mathrm{~s}, \mathrm{C}-8-\mathrm{C}-\mathrm{N})$, $140.63\left(\mathrm{t},{ }^{2} J_{C-F}=22.3 \mathrm{~Hz}, \mathrm{C}-4\right), 137.44(\mathrm{~s}, \mathrm{C}-7), 137.10(\mathrm{~s}, \mathrm{C}-8)$, 131.35 (s, C-6), 121.50 (s, C-5), 121.27 (q, ${ }^{1} \mathrm{~J}_{C-F}=275.3 \mathrm{~Hz}$, $\left.\mathrm{C}(2) \mathrm{CF}_{3}\right), 119.32$ (s, C-5-C-C-4), $113.93-113.73$ (m, C-3), $112.70\left(\mathrm{t},{ }^{1} J_{C-F}=241.7 \mathrm{~Hz}, \mathrm{C}(4) \mathrm{CHF}_{2}\right), 14.91$ (s, Me) ppm. $\mathrm{C}_{12} \mathrm{H}_{7} \mathrm{~F}_{5} \mathrm{NCl}$ (295): calcd (\%) $\mathrm{N} \mathrm{4.73,} \mathrm{C} \mathrm{48.70,} \mathrm{H} \mathrm{2.36,} \mathrm{found} \mathrm{N}$ 4.62, C 48.82, H 2.55 .

2,4-Bis(difluoromethyl)quinoline 3ai. The product was prepared according to the general procedure and starting from an activated solution of TFEDMA 4a (1.2 equiv., $0.427 \mathrm{~mL}, 3.65$ $\mathrm{mmol}$ ) by $\left(\mathrm{BF}_{3} \bullet \mathrm{Et}_{2} \mathrm{O}\right)$ (1.2 equiv., $0.463 \mathrm{~mL}, 3.65 \mathrm{mmol}$ ) and $\mathrm{N}$ (1,1-difluoropropan-2-ylidene)aniline 1aa (1 equiv., $515 \mathrm{mg}$, $3.04 \mathrm{mmol}$ ) in presence of anhydrous acetonitrile. 2,4Bis(difluoromethyl)quinoline $\mathbf{3 a i}$ was provided after purification using a gradient of ethyl acetate in pentane (0-5\%) as a yellow solid $(537 \mathrm{mg}, 77 \%) .{ }^{1} \mathrm{H} \mathrm{NMR} \delta \mathrm{H}=8.23\left(1 \mathrm{H}, \mathrm{d}^{3}{ }^{3} \mathrm{H}_{\mathrm{H}} \mathrm{H}\right.$ $=8.5 \mathrm{~Hz}, \mathrm{C}(8) \mathrm{H}), 8.14\left(1 \mathrm{H}, \mathrm{d},{ }^{3} \mathrm{~J}_{\mathrm{H}-\mathrm{H}}=8.5 \mathrm{~Hz}, \mathrm{C}(5) \mathrm{H}\right), 7.92(1 \mathrm{H}, \mathrm{s}$, $\mathrm{C}(3) \mathrm{H}), 7.86\left(1 \mathrm{H}, \mathrm{t}, 3^{3} \mathrm{H}_{\mathrm{H} H}=7.7 \mathrm{~Hz}, \mathrm{C}(7) \mathrm{H}\right), 7.74\left(1 \mathrm{H}, \mathrm{t},{ }^{3} \mathrm{~J}_{\mathrm{H}-\mathrm{H}}=7.7\right.$ $\mathrm{Hz}, \mathrm{C}(6) \mathrm{H}), 7.19\left(1 \mathrm{H}, \mathrm{t},{ }^{2} \mathrm{~J}_{\mathrm{H}-\mathrm{F}}=54.3 \mathrm{~Hz}, \mathrm{C}\left(4-\mathrm{CHF}_{2}\right) \mathrm{H}\right), 6.81(1 \mathrm{H}, \mathrm{t}$, $\left.{ }^{2} J_{H-F}=55.1 \mathrm{~Hz}, \mathrm{C}\left(2-\mathrm{CHF}_{2}\right) \mathrm{H}\right) \mathrm{ppm} .{ }^{19} \mathrm{~F} \mathrm{NMR} \delta \mathrm{F}=-114.46(2 \mathrm{~F}, \mathrm{~d}$, $\left.2 J_{F-H}=55.1 \mathrm{~Hz}, \mathrm{C} 2-C H F_{2}\right),-115.16\left(2 \mathrm{~F}, \mathrm{~d}, 2 J_{F-H}=54.3 \mathrm{~Hz}, \mathrm{C} 4-\right.$ $\left.\mathrm{CHF}_{2}\right) \mathrm{ppm} .{ }^{13} \mathrm{C}$ NMR $\delta \mathrm{C}=152.70\left(\mathrm{t},{ }^{2} \mathrm{~J}_{\mathrm{C}-\mathrm{F}}=27.1 \mathrm{~Hz}, \mathrm{C}-2\right), 147.80$ (s, C-8-C-N), $139.93\left(\mathrm{t},{ }^{2} J_{C-F}=22.2 \mathrm{~Hz}, \mathrm{C}-4\right), 130.91(\mathrm{~s}, \mathrm{C}-7)$, 130.83 (s, C-8), 129.33 (s, C-6), 124.84 (s, C-5-C-C-4), 123.55 (s, $\mathrm{C}-5), 114.37\left(\mathrm{t},{ }^{1} J_{C-F}=242.0 \mathrm{~Hz}, \mathrm{C}(4) C H F_{2}\right), 114.32-114.17(\mathrm{~m}$, $\mathrm{C}-3), 113.13\left(\mathrm{t},{ }^{1} J_{C-F}=241.2 \mathrm{~Hz}, \mathrm{C}(2) \mathrm{CHF}_{2}\right) \mathrm{ppm} . \mathrm{C}_{11} \mathrm{H}_{7} \mathrm{~F}_{4} \mathrm{~N}(229)$ : calcd (\%) N 6.10, C 57.60, H 3.05, found N 6.20, C 57.56, H 2.96. MP: $48.2-49.7^{\circ} \mathrm{C}$.

4-[Chloro(fluoro)methyl]-2-(difluoromethyl)quinoline 3aii. The product was prepared according to the general procedure and starting from an activated solution of Yarovenko's reagent 4b (1.2 equiv., $0.56 \mathrm{~mL}, 3.55 \mathrm{mmol})$ by $\left(\mathrm{BF}_{3} \bullet \mathrm{Et}_{2} \mathrm{O}\right)$ (1.2 equiv., $0.45 \mathrm{~mL}, \quad 3.55 \mathrm{mmol})$ and $\mathrm{N}$-(1,1-difluoropropan-2ylidene)aniline 1aa (1 equiv., $500 \mathrm{mg}, 2.96 \mathrm{mmol}$ ) in presence of anhydrous acetonitrile. 4-[Chloro(fluoro)methyl]-2(difluoromethyl)quinoline 3aii was provided after purification using a gradient of ethyl acetate in pentane (0-5\%) as a brown solid $(567 \mathrm{mg}, 78 \%) .{ }^{1} \mathrm{H}$ NMR $\delta \mathrm{H}=8.02\left(1 \mathrm{H}, \mathrm{d},{ }^{3} \mathrm{~J}_{\mathrm{H}-\mathrm{H}}=8.5 \mathrm{~Hz}\right.$, $\mathrm{C}(8) \mathrm{H}), 7.88\left(1 \mathrm{H}, \mathrm{d}, 3_{\mathrm{H}-\mathrm{H}}=8.5 \mathrm{~Hz}, \mathrm{C}(5) \mathrm{H}\right), 7.75(1 \mathrm{H}, \mathrm{s}, \mathrm{C}(3) \mathrm{H})$, $7.62\left(1 \mathrm{H}, \mathrm{t},{ }^{3} \mathrm{~J}_{\mathrm{H}-\mathrm{H}}=7.7 \mathrm{~Hz}, \mathrm{C}(7) \mathrm{H}\right), 7.51-7.42(2 \mathrm{H}, \mathrm{m}, \mathrm{C}(7) \mathrm{H} /$ $\mathrm{C}(4-\mathrm{CHFCl}) \mathrm{H}), 6.65\left(1 \mathrm{H}, \mathrm{t},{ }^{2} \mathrm{~J}_{\mathrm{H}-\mathrm{F}}=55.1 \mathrm{~Hz}, \mathrm{C}\left(2-\mathrm{CHF}_{2}\right) \mathrm{H}\right) \mathrm{ppm} .{ }^{19} \mathrm{~F}$ NMR $\delta F=-114.5\left(2 \mathrm{~F}, \mathrm{dd},{ }^{2} J_{F-H}=55.2 \mathrm{~Hz},{ }^{4} J_{F-H}=3 \mathrm{~Hz}, \mathrm{C} 2-\mathrm{CHF}_{2}\right)$, $-138.0\left(1 \mathrm{~F}, \mathrm{~d},{ }^{2} J_{F-H}=49 \mathrm{~Hz}, \mathrm{C} 4-\mathrm{CHFCl}\right) \mathrm{ppm} .{ }^{13} \mathrm{C} \mathrm{NMR} \delta \mathrm{C}=$ $152.63\left(\mathrm{t},{ }^{2} J_{C-F}=27 \mathrm{~Hz}, \mathrm{C}-2\right), 147.69(\mathrm{~s}, \mathrm{C}-8-C-\mathrm{N}), 143.25\left(\mathrm{~d},{ }^{2} J_{C-F}\right.$ $=21 \mathrm{~Hz}, \mathrm{C}-4), 130.80$ (s, C-7), 130.70 (s, C-8), 128.96 (s, C-6), $123.68\left(\mathrm{~d},{ }^{3} \mathrm{~J}_{\mathrm{C}-F}=3.6 \mathrm{~Hz}, \mathrm{C}-5-\mathrm{C}-\mathrm{C}-4\right), 123.06(\mathrm{~s}, \mathrm{C}-5), 114.33$ (t, $\left.{ }^{1} J_{C-F}=241.3 \mathrm{~Hz}, \mathrm{C}(2) \mathrm{CHF}_{2}\right), 113.0\left(\mathrm{dt},{ }^{3} \mathrm{~J}_{C-F}=9.6,{ }^{3} J_{C-F}=1.9 \mathrm{~Hz}, \mathrm{C}-\right.$ 3), $97.08\left(\mathrm{~d},{ }^{1} J_{C-F}=244.3 \mathrm{~Hz}, \mathrm{C}(4) \mathrm{CHFCl}\right) \mathrm{ppm}$. HRMS (ESI +) for $\mathrm{C}_{11} \mathrm{H}_{8} \mathrm{~F}_{3} \mathrm{NCl}[\mathrm{M}+\mathrm{H}]$ : calcd 246.0292, found 246.0312.

2,4-Bis(difluoromethyl)-8-methoxyquinoline $\mathbf{3 b}$. The product was prepared according to the general procedure and starting from an activated solution of TFEDMA $4 a$ (1.2 equiv., $0.354 \mathrm{~mL}$, $3.02 \mathrm{mmol}$ ) by $\left(\mathrm{BF}_{3} \bullet \mathrm{Et}_{2} \mathrm{O}\right)$ (1.2 equiv., $\left.0.383 \mathrm{~mL}, 3.02 \mathrm{mmol}\right)$ and $\mathrm{N}$-(1,1-difluoropropan-2-ylidene)-2-methoxyaniline $\mathbf{1 b b}$ (1 equiv., $502 \mathrm{mg}, 2.52 \mathrm{mmol}$ ) in presence of anhydrous 
acetonitrile. 2,4-Bis(difluoromethyl)-8-methoxyquinoline $\mathbf{3 b}$ was provided after purification using a gradient of ethyl acetate in pentane (10-25\%) as a yellow solid (473 $\mathrm{mg}, 72 \%$ ). ${ }^{1} \mathrm{H} N M R \delta H=7.94(1 \mathrm{H}, \mathrm{s}, \mathrm{C}(5) \mathrm{H}), 7.62\left(2 \mathrm{H}, \mathrm{d},{ }^{3} \mathrm{~J}_{\mathrm{H}-\mathrm{H}}=4.2 \mathrm{~Hz}\right.$, $\mathrm{C}(6,7) \mathrm{H}), 7.31-7.02\left(2 \mathrm{H}, \mathrm{m}, \mathrm{C}(3) \mathrm{H} / \mathrm{C}\left(4-\mathrm{CHF}_{2}\right) \mathrm{H}\right), 6.88(1 \mathrm{H}, \mathrm{t}$, $\left.{ }^{2} J_{H-F}=51.4 \mathrm{~Hz}, \mathrm{C}\left(2-\mathrm{CHF}_{2}\right) \mathrm{H}\right), 4.09(3 \mathrm{H}, \mathrm{s}, \mathrm{Me}) \mathrm{ppm} .{ }^{19} \mathrm{~F} N M R \delta \mathrm{F}$ $=-113.42\left(2 \mathrm{~F}, \mathrm{~d},{ }^{2} J_{F-H}=54.9 \mathrm{~Hz}, \mathrm{C} 2-C H F_{2}\right),-115.88\left(\mathrm{~d},{ }^{2} J_{F-H}=\right.$ $54.3 \mathrm{~Hz}, \mathrm{C} 4-\mathrm{CHF}_{2}$ ) ppm. ${ }^{13} \mathrm{C} \mathrm{NMR} \delta \mathrm{C}=156.17$ (s, C-8), 151.14 (t, $\left.{ }^{2} J_{C-F}=27.7 \mathrm{~Hz}, \mathrm{C}-2\right), 140.04-139.19(2 \mathrm{C}, \mathrm{m}, \mathrm{C}-4 / \mathrm{C}-8-\mathrm{C}-\mathrm{N})$, 129.84 (s, C-6), 126.13 (s, C-5-C-C-4), 114.92 (s, C-5), 114.72 (t, $J=7.9 \mathrm{~Hz}, \mathrm{C}-7), 114.48\left(\mathrm{t},{ }^{1} J_{C-F}=241.9 \mathrm{~Hz}, \mathrm{C}(2) C H F_{2}\right), 112.85(\mathrm{t}$, $\left.{ }^{1} J_{C-F}=241.1 \mathrm{~Hz}, \mathrm{C}(4) C H F_{2}\right), 109.17(\mathrm{~s}, \mathrm{C}-3), 56.41$ (s, Me) ppm. $\mathrm{C}_{12} \mathrm{H}_{9} \mathrm{~F}_{4} \mathrm{NO}$ (259): calcd (\%) N 5.40, C 55.55, H 3.47, found $\mathrm{N}$ 5.35, C 55.30, H 3.51. MP: $96.8-98.6^{\circ} \mathrm{C}$.

2,4-Bis(difluoromethyl)-7-methoxyquinoline 3c. The product was prepared according to the general procedure and starting from an activated solution of TFEDMA 4a (1.2 equiv., $0.356 \mathrm{~mL}$, $3.04 \mathrm{mmol}$ ) by $\left(\mathrm{BF}_{3} \bullet \mathrm{Et}_{2} \mathrm{O}\right)$ (1.2 equiv., $0.386 \mathrm{~mL}, 3.04 \mathrm{mmol}$ ) and $\mathrm{N}$-(1,1-difluoropropan-2-ylidene)-3-methoxyaniline 1cc (1 equiv., $505 \mathrm{mg}, 2.54 \mathrm{mmol}$ ) in presence of anhydrous acetonitrile. 2,4-Bis(difluoromethyl)-7-methoxyquinoline $3 c$ was provided after purification using a gradient of ethyl acetate in pentane (10-25\%) as a yellow solid $(515 \mathrm{mg}, 78 \%)$. $1 \mathrm{H} N M R \delta \mathrm{H}=7.79\left(1 \mathrm{H}, \mathrm{d},{ }^{3} J_{\mathrm{H}-\mathrm{H}}=9.3 \mathrm{~Hz}, \mathrm{C}(5) \mathrm{H}\right), 7.57(1 \mathrm{H}, \mathrm{s}$, $\mathrm{C}(3) \mathrm{H}), 7.30\left(1 \mathrm{H}, \mathrm{d},{ }^{4} J_{H-H}=2.6 \mathrm{~Hz}, \mathrm{C}(8) \mathrm{H}\right), 7.14\left(1 \mathrm{H}, \mathrm{dd},{ }^{3} J_{H-H}=\right.$ 9.3, $\left.{ }^{4} J_{H-H}=2.6 \mathrm{~Hz}, \mathrm{C}(6) \mathrm{H}\right), 6.94\left(1 \mathrm{H}, \mathrm{t},{ }^{2} J_{\mathrm{H}-\mathrm{F}}=54.4 \mathrm{~Hz}, \mathrm{C}(4-\right.$ $\left.\left.\mathrm{CHF}_{2}\right) \mathrm{H}\right), 6.62\left(1 \mathrm{H}, \mathrm{t}^{2}{ }^{2} \mathrm{H}_{-F}=55.2 \mathrm{~Hz}, \mathrm{C}\left(2-\mathrm{CHF}_{2}\right) \mathrm{H}\right), 3.79(3 \mathrm{H}, \mathrm{s}$, Me) ppm. ${ }^{19} \mathrm{~F} N M R \delta F=-114.79\left(2 \mathrm{~F}, \mathrm{~d},{ }^{2} J_{F-H}=54.4 \mathrm{~Hz}, \mathrm{C} 2-\right.$ $\left.\mathrm{CHF}_{2}\right),-114.95\left(2 \mathrm{~F}, \mathrm{~d},{ }^{2} \mathrm{~J}_{F-H}=55.3 \mathrm{~Hz}, \mathrm{C} 4-\mathrm{CHF}_{2}\right) \mathrm{ppm} .{ }^{13} \mathrm{C} \mathrm{NMR}$ $\delta \mathrm{C}=161.45(\mathrm{~s}, \mathrm{C}-7), 152.86\left(\mathrm{t},{ }^{2} J_{\mathrm{C}-F}=26.7 \mathrm{~Hz}, \mathrm{C}-2\right), 149.83(\mathrm{~s}, \mathrm{C}-$ 8-C-N), $139.56\left(\mathrm{t},{ }^{2} J_{C-F}=22.2 \mathrm{~Hz}, \mathrm{C}-4\right), 124.33(\mathrm{~s}, \mathrm{C}-5), 122.45$ (s, $\mathrm{C}-6), 119.77\left(\mathrm{t},{ }^{3} \mathrm{~J}_{\mathrm{C}-\mathrm{F}}=2.8 \mathrm{~Hz}, \mathrm{C}-5-\mathrm{C}-\mathrm{C}-4\right), 114.25\left(\mathrm{t},{ }^{1} \mathrm{~J}_{\mathrm{C}-F}=242.4\right.$ $\left.\mathrm{Hz}, \mathrm{C}(2) C H F_{2}\right), 113.18\left(\mathrm{t},{ }^{1} \mathrm{~J}_{\mathrm{C}-F}=241.0 \mathrm{~Hz}, \mathrm{C}(4) \mathrm{CHF}_{2}\right), 111.89-$ 111.70 (m, C-3), 108.24 (s, C-8), 55.60 (s, Me) ppm. $\mathrm{C}_{12} \mathrm{H}_{9} \mathrm{~F}_{4} \mathrm{NO}$ (259): calcd (\%) N 5.40, C 55.55, H 3.47, found N 5.55, C 55.57, H 3.67. MP: $55.4-56.9^{\circ} \mathrm{C}$.

2,4-Bis(difluoromethyl)-6-methoxyquinoline 3d. The product was prepared according to the general procedure and starting from an activated solution of TFEDMA 4a (1.2 equiv., $0.357 \mathrm{~mL}$, $3.05 \mathrm{mmol})$ by $\left(\mathrm{BF}_{3} \bullet \mathrm{Et}_{2} \mathrm{O}\right)$ (1.2 equiv., $0.386 \mathrm{~mL}, 3.05 \mathrm{mmol}$ ) and $\mathrm{N}$-(1,1-difluoropropan-2-ylidene)-4-methoxyaniline 1dd (1 equiv., $506 \mathrm{mg}, 2.54 \mathrm{mmol}$ ) in presence of anhydrous acetonitrile. 2,4-Bis(difluoromethyl)-6-methoxyquinoline 3d was provided after purification using a gradient of ethyl acetate in pentane (10-25\%) as an orange solid (468 $\mathrm{mg}, 71 \%$ ). ${ }^{1} \mathrm{H} N M R \delta \mathrm{H}=8.15\left(1 \mathrm{H}, \mathrm{d},{ }^{3} \mathrm{~J}_{\mathrm{H}-\mathrm{H}}=9.3 \mathrm{~Hz}, \mathrm{C}(8) \mathrm{H}\right), 7.90(1 \mathrm{H}, \mathrm{s}$, $\mathrm{C}(3) \mathrm{H}), 7.53\left(1 \mathrm{H}, \mathrm{dd},{ }^{3} J_{H-H}=9.3,{ }^{4} J_{H-H}=2.5 \mathrm{~Hz}, \mathrm{C}(7) \mathrm{H}\right), 7.36(1$ $\mathrm{H}, \mathrm{s}, \mathrm{C}(5) \mathrm{H}), 7.16\left(1 \mathrm{H}, \mathrm{t},{ }^{2} \mathrm{~J}_{\mathrm{H}-\mathrm{F}}=54.4 \mathrm{~Hz}, \mathrm{C}\left(4-\mathrm{CHF}_{2}\right) \mathrm{H}\right), 6.85(1 \mathrm{H}$, $\left.\mathrm{t},{ }^{2} \mathrm{~J}_{\mathrm{H}-\mathrm{F}}=55.2 \mathrm{~Hz}, \mathrm{C}\left(2-\mathrm{CHF}_{2}\right) \mathrm{H}\right), 4.03(3 \mathrm{H}, \mathrm{s}, \mathrm{Me}) \mathrm{ppm} .{ }^{19} \mathrm{~F} \mathrm{NMR}$ $\delta \mathrm{F}=-113.99\left(2 \mathrm{~F}, \mathrm{~d}, 2 J_{F-H}=55.3 \mathrm{~Hz}, \mathrm{C} 2-\mathrm{CHF}_{2}\right),-115.52(2 \mathrm{~F}, \mathrm{~d}$, $\left.2 J_{F-H}=54.4 \mathrm{~Hz}, \mathrm{C} 4-\mathrm{CHF}_{2}\right) \mathrm{ppm} .{ }^{13} \mathrm{C} \mathrm{NMR} \delta \mathrm{C}=159.77(\mathrm{~s}, \mathrm{C}-6)$, $149.93\left(\mathrm{t},{ }^{2} J_{C-F}=27.0 \mathrm{~Hz}, \mathrm{C}-2\right), 143.966(\mathrm{~s}, \mathrm{C}-8-\mathrm{C}-\mathrm{N}), 138.02(\mathrm{t}$, $\left.{ }^{2} J_{C-F}=22.0 \mathrm{~Hz}, \mathrm{C}-4\right), 132.11(\mathrm{~s}, \mathrm{C}-8), 126.21$ (s, C-5-C-C-4), $123.71(\mathrm{~s}, \mathrm{C}-7), 114.70\left(\mathrm{tt}, 3^{3} \mathrm{C}_{\mathrm{F} F}=8.0,{ }^{3} \mathrm{~J}_{\mathrm{C}-F}=1.8 \mathrm{~Hz}, \mathrm{C}-3\right), 114.51$ (t, C(2)CHF $\left.{ }^{1} J_{C-F}=241.9 \mathrm{~Hz}\right), 113.57\left(\mathrm{t}, \mathrm{C}(4) \mathrm{CHF}_{2},{ }^{1} J_{C-F}=240.8\right.$ $\mathrm{Hz}$ ), 101.35 (s, C-5), 55.79 (s, Me) ppm. $\mathrm{C}_{12} \mathrm{H}_{9} \mathrm{~F}_{4} \mathrm{NO}$ (259): calcd
(\%) N 5.40, C 55.55, H 3.47, found N 5.43, C 55.24, H 3.30. MP: $93.5-97.2^{\circ} \mathrm{C}$.

2,4-Bis(difluoromethyl)-8-fluoroquinoline $3 e$. The product was prepared according to the general procedure and starting from an activated solution of TFEDMA $4 a$ (1.2 equiv., $0.377 \mathrm{~mL}$, $3.22 \mathrm{mmol})$ by $\left(\mathrm{BF}_{3} \bullet \mathrm{Et}_{2} \mathrm{O}\right)$ (1.2 equiv., $0.408 \mathrm{~mL}, 3.22 \mathrm{mmol}$ ) and $N$-(1,1-difluoropropan-2-ylidene)-2-fluoroaniline 1ee (1 equiv., $502 \mathrm{mg}, 2.68 \mathrm{mmol}$ ) in presence of anhydrous acetonitrile. 2,4-Bis(difluoromethyl)-8-fluoroquinoline $\mathbf{3 e}$ was provided after purification using a gradient of ethyl acetate in pentane $(0-5 \%)$ as a yellow solid $(466 \mathrm{mg}, 39 \%)$ in mixture (3: 1) with the 1,1,5,5-tetrafluoro-4-((2-fluorophenyl)amino)pent3-en-2-one after purification $\left(6^{\prime} \mathbf{e}\right) .{ }^{1} \mathrm{H} N M R \delta \mathrm{H}=8.00(1 \mathrm{H}, \mathrm{s}$, $\mathrm{C}(3) \mathrm{H}), 7.93\left(1 \mathrm{H}, \mathrm{d},{ }^{3} \mathrm{~J}_{\mathrm{H}-\mathrm{H}}=8.6 \mathrm{~Hz}, \mathrm{C}(5) \mathrm{H}\right), 7.73-7.68(1 \mathrm{H}, \mathrm{m}$, $\mathrm{C}(6) \mathrm{H}), 7.60-7.52(1 \mathrm{H}, \mathrm{m}, \mathrm{C}(7) \mathrm{H}), 7.17\left(1 \mathrm{H}, \mathrm{t},{ }^{2} J_{H-F}=54.2 \mathrm{~Hz}\right.$, $\left.\mathrm{C}\left(4-\mathrm{CHF}_{2}\right) \mathrm{H}\right), 6.86\left(1 \mathrm{H}, \mathrm{t},{ }^{2} J_{\mathrm{H}-\mathrm{F}}=54.8 \mathrm{~Hz}, \mathrm{C}\left(2-\mathrm{CHF}_{2}\right) \mathrm{H}\right) \mathrm{ppm}$. HRMS $(E S I+)$ for $\mathrm{C}_{11} \mathrm{H}_{7} \mathrm{~F}_{5} \mathrm{~N}[\mathrm{M}+\mathrm{H}]$ : calcd 248.0493, found 248.0499 .

2,4-Bis(difluoromethyl)-7-fluoroquinoline 3f. The product was prepared according to the general procedure and starting from an activated solution of TFEDMA 4a (1.2 equiv., $0.376 \mathrm{~mL}, 3.22$ $\mathrm{mmol})$ by $\left(\mathrm{BF}_{3} \bullet \mathrm{Et}_{2} \mathrm{O}\right)$ (1.2 equiv., $0.408 \mathrm{~mL}, 3.22 \mathrm{mmol}$ ) and $\mathrm{N}$ (1,1-difluoropropan-2-ylidene)-3-fluoroaniline 1 ff (1 equiv., $501 \mathrm{mg}, 2.68 \mathrm{mmol}$ ) in presence of anhydrous acetonitrile. 2,4Bis(difluoromethyl)-7-fluoroquinoline $\mathbf{3 f}$ was provided after purification using a gradient of ethyl acetate in pentane (0-5\%) as a yellow solid (464 mg, 70\%). ${ }^{1} \mathrm{H} \mathrm{NMR} \delta \mathrm{H}=8.17\left(1 \mathrm{H}, \mathrm{dd},{ }^{4} J_{\mathrm{H}-}\right.$ $\left.H=9.3,{ }^{3} J_{H-F}=5.7 \mathrm{~Hz}, \mathrm{C}(8) \mathrm{H}\right), 7.86(1 \mathrm{H}, \mathrm{s}, \mathrm{C}(3) \mathrm{H}), 7.84(1 \mathrm{H}, \mathrm{s}$, $\mathrm{C}(5) \mathrm{H}), 7.57-7.49(1 \mathrm{H}, \mathrm{m}, \mathrm{C}(6) \mathrm{H}), 7.14\left(1 \mathrm{H}, \mathrm{t},{ }^{2} J_{\mathrm{H}-\mathrm{F}}=54.2 \mathrm{~Hz}\right.$, $\left.\mathrm{C}\left(4-\mathrm{CHF}_{2}\right) \mathrm{H}\right), 6.78\left(1 \mathrm{H}, \mathrm{t}^{2}{ }^{2} \mathrm{~J}_{\mathrm{H}-\mathrm{F}}=55.0 \mathrm{~Hz}, \mathrm{C}\left(2-\mathrm{CHF}_{2}\right) \mathrm{H}\right) \mathrm{ppm} .{ }^{19} \mathrm{~F}$ NMR $\delta F=-106.41--106.96(1 \mathrm{~F}, \mathrm{~m}, \mathrm{~F}),-114.43\left(2 \mathrm{~F}, \mathrm{~d},{ }^{2} J_{F-H}=\right.$ $\left.54.2 \mathrm{~Hz}, \mathrm{C} 2-\mathrm{CHF}_{2}\right),-114.83\left(2 \mathrm{~F}, \mathrm{~d}, 2 \mathrm{~J}_{\mathrm{F}-\mathrm{H}}=55.0 \mathrm{~Hz}, \mathrm{C} 4-\mathrm{CHF}_{2}\right)$ ppm. ${ }^{13} \mathrm{C} N M R \delta C=163.60\left(\mathrm{~d},{ }^{1} J_{C-F}=253.6 \mathrm{~Hz}, \mathrm{C}-7\right), 153.93(\mathrm{t}$, $\left.{ }^{2} J_{C-F}=27.3 \mathrm{~Hz}, \mathrm{C}-2\right), 149.21\left(\mathrm{~d},{ }^{3} J_{C-F}=12.7 \mathrm{~Hz}, \mathrm{C}-8-C-\mathrm{N}\right), 140.17$ $\left(\mathrm{td},{ }^{2} J_{C-F}=22.4,{ }^{5} J_{C-F}=1.1 \mathrm{~Hz}, \mathrm{C}-4\right), 126.00\left(\mathrm{~d},{ }^{3} J_{C-F}=9.8 \mathrm{~Hz}, \mathrm{C}-5\right)$, $121.79\left(\mathrm{~d},{ }^{4} J_{C-F}=1.0 \mathrm{~Hz}, \mathrm{C}-5-C-C-4\right), 119.91\left(\mathrm{~d},{ }^{2} J_{C-F}=25.39 \mathrm{~Hz}\right.$, C-6), $114.54\left(\mathrm{~d},{ }^{2} J_{C-F}=20.56 \mathrm{~Hz}, \mathrm{C}-8\right), 114.12\left(\mathrm{t},{ }^{1} J_{C-F}=242.9 \mathrm{~Hz}\right.$, $\left.\mathrm{C}(2) \mathrm{CHF}_{2}\right), 113.86(\mathrm{~m}, \mathrm{C}-3), 113.23\left(\mathrm{t},{ }^{1} \mathrm{~J}_{\mathrm{C}-\mathrm{F}}=241.5 \mathrm{~Hz}, \mathrm{C}(4) \mathrm{CHF}_{2}\right)$ ppm. $\mathrm{C}_{11} \mathrm{H}_{6} \mathrm{~F}_{5} \mathrm{~N}$ (247): calcd (\%) N 5.66, C 53.41, H 2.43, found $\mathrm{N}$ 5.79, C 53.54, H 2.69. MP: $73.2-74.6^{\circ} \mathrm{C}$.

2,4-Bis(difluoromethyl)-6-fluoroquinoline 3gi. The product was prepared according to the general procedure and starting from an activated solution of TFEDMA 4 a (1.5 equiv., $0.375 \mathrm{~mL}$, $3.2 \mathrm{mmol}$ ) by $\left(\mathrm{BF}_{3} \bullet \mathrm{Et}_{2} \mathrm{O}\right.$ ) (1.51 equiv., $0.408 \mathrm{~mL}, 3.22 \mathrm{mmol}$ ) and $\mathrm{N}$-(1,1-difluoropropan-2-ylidene)-4-fluoroaniline 1gg (1 equiv., $400 \mathrm{mg}, 2.14 \mathrm{mmol}$ ) in presence of anhydrous acetonitrile. 2,4-Bis(difluoromethyl)-6-fluoroquinoline 3gi was provided after purification using a gradient of ethyl acetate in pentane (0-5\%) as a yellow solid (205 mg, 39\%). ${ }^{1} \mathrm{H} \mathrm{NMR} \delta \mathrm{H}=$ $8.24\left(1 \mathrm{H}, \mathrm{dd},{ }^{3} J_{H-H}=9.3,{ }^{4} J_{H-F}=5.5 \mathrm{~Hz}, \mathrm{C}(8) \mathrm{H}\right), 7.92(1 \mathrm{H}, \mathrm{s}$, $\mathrm{C}(3) \mathrm{H}), 7.80-7.74(1 \mathrm{H}, \mathrm{m}, \mathrm{C}(5) \mathrm{H}), 7.65-7.60(1 \mathrm{H}, \mathrm{m}, \mathrm{C}(7) \mathrm{H})$, $7.09\left(1 \mathrm{H}, \mathrm{t},{ }^{2} \mathrm{~J}_{\mathrm{H}-\mathrm{F}}=54.2 \mathrm{~Hz}, \mathrm{C}\left(4-\mathrm{CHF}_{2}\right) \mathrm{H}\right), 6.79\left(1 \mathrm{H}, \mathrm{t},{ }^{2} \mathrm{~J}_{\mathrm{H}-\mathrm{F}}=55.0\right.$ $\left.\mathrm{Hz}, \mathrm{C}\left(2-\mathrm{CHF}_{2}\right) \mathrm{H}\right) \mathrm{ppm} .{ }^{19} \mathrm{~F} N M R \delta \mathrm{F}=-107.18--107.24(1 \mathrm{~F}, \mathrm{~m}$, F), $-114.45\left(2 \mathrm{~F}, \mathrm{~d}, 2 J_{F-H}=55.0 \mathrm{~Hz}, \mathrm{C} 2-\mathrm{CHF}_{2}\right),-115.17\left(2 \mathrm{~F}, \mathrm{~d},{ }^{2} J_{F-H}\right.$ $\left.=54.2 \mathrm{~Hz}, \mathrm{C} 4-\mathrm{CHF}_{2}\right) \mathrm{ppm} .{ }^{13} \mathrm{C} \mathrm{NMR} \delta \mathrm{C}=162.01\left(\mathrm{~d},{ }^{1} J_{\mathrm{C}-F}=252.9\right.$ $\mathrm{Hz}, \mathrm{C}-6), 152.09\left(\mathrm{td},{ }^{2} J_{C-F}=27.3,{ }^{6} J_{C-F}=3.1 \mathrm{~Hz}, \mathrm{C}-2\right), 144.98(\mathrm{~s}, \mathrm{C}-$ $8-C-\mathrm{N}), 139.56\left(\mathrm{td},{ }^{2} J_{C-F}=22.4,{ }^{4} J_{C-F}=6.1 \mathrm{~Hz}, \mathrm{C}-4\right), 133.46\left(\mathrm{~d},{ }^{3} J_{C}\right.$ 
$F=9.6 \mathrm{~Hz}, \mathrm{C}-8), 125.76\left(\mathrm{~d},{ }^{3} J_{C-F}=10.4 \mathrm{~Hz}, \mathrm{C}-5-C-C-4\right), 121.43(\mathrm{~d}$, $\left.{ }^{2} J_{C-F}=25.8 \mathrm{~Hz}, \mathrm{C}-7\right), 115.28\left(\mathrm{t},{ }^{3} J_{C-F}=7.8 \mathrm{~Hz}, \mathrm{C}-3\right), 114.21\left(\mathrm{t},{ }^{1} J_{C-F}\right.$ $\left.=241.3 \mathrm{~Hz}, \mathrm{C}(2) C H F_{2}\right), 110.78\left(\mathrm{t},{ }^{1} J_{C-F}=242.0 \mathrm{~Hz}, \mathrm{C}(4) C H F_{2}\right)$, $107.84\left(d,{ }^{2} J_{C-F}=24.0 \mathrm{~Hz}, \mathrm{C}-5\right)$ ppm. $\mathrm{C}_{11} \mathrm{H}_{6} \mathrm{~F}_{5} \mathrm{~N}(247)$ : calcd (\%) N 5.66, C 53.41, H 2.43, found N 5.67, C 53.42, H 2.57. HRMS (ESI + ) for $\mathrm{C}_{11} \mathrm{H}_{7} \mathrm{~F}_{5} \mathrm{~N}[\mathrm{M}+\mathrm{H}]$ : calcd 248.0493, found 248.0497. MP: $68.7-71.2^{\circ} \mathrm{C}$.

\section{4-[Chloro(fluoro)methyl]-2-(difluoromethyl)-6-}

fluoroquinoline 3gii. The product was prepared according to the general procedure and starting from an activated solution of Yarovenko's reagent 4b (1.2 equiv., $0.597 \mathrm{~mL}, 2.62 \mathrm{mmol}$ ) by $\left(\mathrm{BF}_{3} \bullet \mathrm{Et}_{2} \mathrm{O}\right)$ (1.2 equiv., $\left.0.332 \mathrm{~mL}, 2.62 \mathrm{mmol}\right)$ and $\mathrm{N}-(1,1-$ difluoropropan-2-ylidene)-4-fluoroaniline 1gg (1 equiv., 409 $\mathrm{mg}, 2.19 \mathrm{mmol}$ ) in presence of anhydrous acetonitrile. 4[Chloro(fluoro)methyl]-2-(difluoromethyl)-6-fluoroquinoline 3gii was provided after purification using a gradient of ethyl acetate in pentane (0-5\%) as a brown liquid $(17 \mathrm{mg}, 3 \%$ estimated). ${ }^{1} \mathrm{H} N M R \delta H=8.26\left(1 \mathrm{H}, \mathrm{dd},{ }^{3} J_{H-H}=9.3,{ }^{4} J_{H-F}=5.5\right.$ $\mathrm{Hz}, \mathrm{C}(8) \mathrm{H}), 7.94(1 \mathrm{H}, \mathrm{s}, \mathrm{C}(3) \mathrm{H}), 7.77\left(1 \mathrm{H}, \mathrm{dd},{ }^{3} J_{H-F}=9.6,{ }^{4} J_{H-H}=\right.$ $2.7 \mathrm{~Hz}, \mathrm{C}(5) \mathrm{H}), 7.63\left(1 \mathrm{H}, \mathrm{ddd},{ }^{3} J_{H-H}=9.3,{ }^{4} J_{H-F}=8.0,{ }^{4} J_{H-H}=2.7\right.$ $\mathrm{Hz}, \mathrm{C}(7) \mathrm{H}), 7.53\left(1 \mathrm{H}, \mathrm{d},{ }^{2} J_{H-F}=48.9 \mathrm{~Hz}, \mathrm{C}(4-\mathrm{CHFCl}) \mathrm{H}\right), 6.80(1 \mathrm{H}$, $\left.\mathrm{t},{ }^{2} \mathrm{~J}_{\mathrm{H}-\mathrm{F}}=55.0 \mathrm{~Hz}, \mathrm{C}\left(2-\mathrm{CHF}_{2}\right) \mathrm{H}\right) \mathrm{ppm} .{ }^{19} \mathrm{~F} \mathrm{NMR} \delta \mathrm{F}=-106.78-$ $106.84(1 \mathrm{~F}, \mathrm{~m}, \mathrm{~F}),-114.49\left(2 \mathrm{~F}, \mathrm{dd}, 2 \mathrm{~J}_{F-H}=55.0,4 J_{F-H}=2.9 \mathrm{~Hz}\right.$, $\left.\mathrm{C} 2-\mathrm{CHF}_{2}\right),-137.65\left(1 \mathrm{~F}, \mathrm{~d},{ }^{2} J_{F-H}=48.7 \mathrm{~Hz}, \mathrm{C} 4-\mathrm{CHFCl}\right) \mathrm{ppm} .{ }^{13} \mathrm{C}$ NMR $\delta C=161.61\left(\mathrm{~d},{ }^{1} J_{C-F}=253.2 \mathrm{~Hz}, \mathrm{C}-6\right), 152.11\left(\mathrm{t},{ }^{2} J_{C-F}=27.1\right.$ $\mathrm{Hz}, \mathrm{C}-2$ ), 144.94 (s, C-8-C-N), 143.01 (dd, ${ }^{2} J_{C-F}=20.9,{ }^{4} J_{C-F}=6.2$ $\mathrm{Hz}, \mathrm{C}-4), 133.56$ (d, $\left.{ }^{3} \mathrm{C}_{\mathrm{C}-F}=9.7 \mathrm{~Hz}, \mathrm{C}-8\right), 128.42$ (s, C-5-C-C-4), $121.43\left(\mathrm{~d},{ }^{2} J_{C-F}=25.8 \mathrm{~Hz}, \mathrm{C}-7\right), 114.11\left(\mathrm{t},{ }^{1} J_{C-F}=241.0 \mathrm{~Hz}\right.$, $\left.\mathrm{C}(2) C H F_{2}\right), 114.10\left(\mathrm{~d},{ }^{3} J_{C-F}=8.8 \mathrm{~Hz}, \mathrm{C}-3\right), 107.65\left(\mathrm{~d},{ }^{2} J_{C-F}=24.1\right.$ $\mathrm{Hz}, \mathrm{C}-5), 97.09\left(\mathrm{~d},{ }^{1} J_{C-F}=244.8 \mathrm{~Hz}, \mathrm{C}(4) \mathrm{CHFCl}\right.$ ) ppm. HRMS (ESI positive) for $\mathrm{C}_{11} \mathrm{H}_{7} \mathrm{~F}_{4} \mathrm{NCl}[\mathrm{M}+\mathrm{H}]$ : calcd 264.0198, found 264.0198.

2,4-Bis(difluoromethyl)-8-(trifluoromethoxy)quinoline $3 \mathrm{~h}$. The product was prepared according to the general procedure and starting from an activated solution of TFEDMA 4a (1.44 equiv., $0.34 \mathrm{~mL}, 2.91 \mathrm{mmol})$ by $\left(\mathrm{BF}_{3} \bullet \mathrm{Et}_{2} \mathrm{O}\right)$ (1.44 equiv., 0.37 $\mathrm{mL}, 2.92 \mathrm{mmol}$ ) and $\mathrm{N}$-(1,1-difluoropropan-2-ylidene)-2(trifluoromethoxy)aniline $1 \mathrm{hh}$ (1 equiv., $512 \mathrm{mg}, 2.02 \mathrm{mmol}$ ) in presence of anhydrous acetonitrile. 2,4-Bis(difluoromethyl)-8(trifluoromethoxy)quinoline $\mathbf{3 h}$ was provided after purification using a gradient of ethyl acetate in pentane (0-5\%) as a yellow solid $(244 \mathrm{mg}, 39 \%) .{ }^{1} \mathrm{H} N M R \delta H=8.11\left(1 \mathrm{H}, \mathrm{dd},{ }^{3} J_{H-H}=8.1,{ }^{4} J_{H-}\right.$ $\left.{ }_{H}=1.4 \mathrm{~Hz}, \mathrm{C}(7) \mathrm{H}\right), 8.01(1 \mathrm{H}, \mathrm{s}, \mathrm{C}(3) \mathrm{H}), 7.76-7.75(2 \mathrm{H}, \mathrm{m}$, $\mathrm{C}(5,6) \mathrm{H}), 7.18\left(1 \mathrm{H}, \mathrm{t},{ }^{2} J_{\mathrm{H}-F}=54.1 \mathrm{~Hz}, \mathrm{C}\left(4-\mathrm{CHF}_{2}\right) \mathrm{H}\right), 6.85(1 \mathrm{H}, \mathrm{t}$, $\left.{ }^{2} J_{H-F}=54.8 \mathrm{~Hz}, \mathrm{C}\left(2-\mathrm{CHF}_{2}\right) \mathrm{H}\right) \mathrm{ppm} .{ }^{19} \mathrm{~F} \mathrm{NMR} \delta \mathrm{F}=-57.55(3 \mathrm{~F}, \mathrm{~s}$, $\left.\mathrm{OCF}_{3}\right),-114.12\left(2 \mathrm{~F}, \mathrm{~d},{ }^{2} \mathrm{~J}_{F-H}=54.9 \mathrm{~Hz}, \mathrm{C} 2-\mathrm{CHF}_{2}\right),-114.91(2 \mathrm{~F}, \mathrm{~d}$, $\left.2 J_{F-H}=54.3 \mathrm{~Hz}, \mathrm{C} 4-\mathrm{CHF}_{2}\right) \mathrm{ppm} .{ }^{13} \mathrm{C} \mathrm{NMR} \delta \mathrm{C}=153.55\left(\mathrm{t},{ }^{2} J_{\mathrm{C}-F}=\right.$ $28.0 \mathrm{~Hz}, \mathrm{C}-2), 146.06$ (s, C-8), 141.11 (s, C-8-C-N), 140.23 (t, ${ }^{2} J_{C-F}$ $=22.5 \mathrm{~Hz}, \mathrm{C}-4), 128.98(\mathrm{~s}, \mathrm{C}-5),$,126.25 (s, C-5-C-C-4), 122.56 (s, $\mathrm{C}-7$, ), 122.14 (s, C-6), $120.47\left(\mathrm{q},{ }^{1} J_{C-F}=259.6 \mathrm{~Hz}, \mathrm{OCF}_{3}\right), 115.59$ $\left(\mathrm{t},{ }^{3} J_{C-F}=7.9 \mathrm{~Hz}, \mathrm{C}-3\right), 114.29\left(\mathrm{t},{ }^{1} J_{C-F}=242.4 \mathrm{~Hz}, \mathrm{C}(2) C H F_{2}\right)$, $112.98\left(\mathrm{t},{ }^{1} J_{C-F}=241.7 \mathrm{~Hz}, \mathrm{C}(4) C H F_{2}\right) \mathrm{ppm} . \mathrm{C}_{12} \mathrm{H}_{6} \mathrm{~F}_{7} \mathrm{NO}(313)$ : calcd (\%) N 4.47, C 45.98, H 1.92, found N 4.57, C 46.23, H 1.98. MP: $59.4-60^{\circ} \mathrm{C}$.

2,4-Bis(difluoromethyl)-7-(trifluoromethoxy)quinoline 3i. The product was prepared according to the general procedure and starting from an activated solution of TFEDMA 4 a (1.2 equiv.,
$0.278 \mathrm{~mL}, 2.38 \mathrm{mmol}$ ) by $\left(\mathrm{BF}_{3} \bullet \mathrm{Et}_{2} \mathrm{O}\right.$ ) (1.2 equiv., $0.301 \mathrm{~mL}, 2.38$ $\mathrm{mmol})$ and $\mathrm{N}$-(1,1-difluoropropan-2-ylidene)-3(trifluoromethoxy)aniline 1ii (1 equiv., $501 \mathrm{mg}, 1.98 \mathrm{mmol}$ ) in presence of anhydrous acetonitrile. 2,4-Bis(difluoromethyl)-7(trifluoromethoxy)quinoline $\mathbf{3 i}$ was provided after purification using a gradient of ethyl acetate in pentane (0-5\%) as a light brown liquid (440 mg, 71\%). ${ }^{1} \mathrm{H}$ NMR $\delta \mathrm{H}=8.08\left(1 \mathrm{H}, \mathrm{d}^{3}{ }^{3} \mathrm{~J}_{\mathrm{H}-\mathrm{H}}=\right.$ $9.2 \mathrm{~Hz}, \mathrm{C}(5) \mathrm{H}), 7.93(1 \mathrm{H}, \mathrm{s}, \mathrm{C}(8) \mathrm{H}), 7.79(1 \mathrm{H}, \mathrm{s}, \mathrm{C}(3) \mathrm{H}), 7.46(1$ $\left.\mathrm{H}, \mathrm{d}, 3^{3} \mathrm{H}_{-\mathrm{H}}=9.2 \mathrm{~Hz}, \mathrm{C}(6) \mathrm{H}\right), 7.03\left(1 \mathrm{H}, \mathrm{t},{ }^{2} \mathrm{~J}_{\mathrm{H}-\mathrm{F}}=54.2 \mathrm{~Hz}, \mathrm{C}(4-\right.$ $\left.\left.\mathrm{CHF}_{2}\right) \mathrm{H}\right), 6.69\left(1 \mathrm{H}, \mathrm{t},{ }^{2} \mathrm{~J}_{\mathrm{H}-\mathrm{F}}=55.0 \mathrm{~Hz}, \mathrm{C}\left(2-\mathrm{CHF}_{2}\right) \mathrm{H}\right) \mathrm{ppm} .{ }^{19} \mathrm{~F} \mathrm{NMR}$ $\delta \mathrm{F}=-58.14\left(3 \mathrm{~F}, \mathrm{~s}, \mathrm{OCF}_{3}\right),-114.72\left(2 \mathrm{~F}, \mathrm{~d},{ }^{2} \mathrm{~J}_{F-H}=54.1 \mathrm{~Hz}, \mathrm{C} 2-\right.$ $\left.\mathrm{CHF}_{2}\right),-115.17\left(2 \mathrm{~F}, \mathrm{~d},{ }^{2} \mathrm{~J}_{F-H}=54.9 \mathrm{~Hz}, \mathrm{C} 4-\mathrm{CHF}_{2}\right) \mathrm{ppm} .{ }^{13} \mathrm{C} \mathrm{NMR}$ $\delta \mathrm{C}=154.12\left(\mathrm{t},{ }^{2} J_{C-F}=27.3 \mathrm{~Hz}, \mathrm{C}-2\right), 150.58(\mathrm{~s}, \mathrm{C}-7), 148.46(\mathrm{~s}, \mathrm{C}-$ 8-C-N), $140.14\left(\mathrm{t},{ }^{2} J_{C-F}=22.5 \mathrm{~Hz}, \mathrm{C}-4\right), 125.76(\mathrm{~s}, \mathrm{C}-5), 123.13$ (s, C-6), 122.95 (s, C-5-C-C-4), 120.58 (q, ${ }^{1} J_{C-F}=259.4 \mathrm{~Hz}, \mathrm{OCF}_{3}$ ), $120.02(\mathrm{~s}, \mathrm{C}-8), 114.72\left(\mathrm{tt},{ }^{3} J_{C-F}=8,{ }^{3} J_{C-F}=1.8 \mathrm{~Hz}, \mathrm{C}-3\right), 114.04$ $\left(t,{ }^{1} J_{C-F}=242.4 \mathrm{~Hz}, \mathrm{C}(2) C H F_{2}\right), 113.12\left(\mathrm{t},{ }^{1} J_{C-F}=241.4 \mathrm{~Hz}\right.$, $\mathrm{C}(4) \mathrm{CHF}_{2}$ ) ppm. $\mathrm{C}_{12} \mathrm{H}_{6} \mathrm{~F}_{7} \mathrm{NO}$ (313): calcd (\%) N 4.47, C 45.98, H 1.92 , found N 4.49, C 46.13, H 2.15 .

2,4-Bis(difluoromethyl)-6-(trifluoromethoxy)quinoline $\mathbf{3 j}$. The product was prepared according to the general procedure and starting from an activated solution of TFEDMA 4a (1.5 equiv., $0.347 \mathrm{~mL}, 2.96 \mathrm{mmol}$ ) by $\left(\mathrm{BF}_{3} \bullet \mathrm{Et}_{2} \mathrm{O}\right)$ (1.51 equiv., $0.378 \mathrm{~mL}$, $2.98 \mathrm{mmol})$ and $\mathrm{N}$-(1,1-difluoropropan-2-ylidene)-4(trifluoromethoxy)aniline $\mathbf{1 j j}$ (1 equiv., $500 \mathrm{mg}, 1.98 \mathrm{mmol}$ ) in presence of anhydrous acetonitrile. 2,4-Bis(difluoromethyl)-6(trifluoromethoxy)quinoline $\mathbf{3 j}$ was provided after purification using a gradient of ethyl acetate in pentane (0-5\%) as a yellow solid (361 mg, 58\%). ${ }^{1} \mathrm{H} N M R \delta H=8.30\left(1 \mathrm{H}, \mathrm{d},{ }^{3} \mathrm{~J}_{\mathrm{H}-\mathrm{H}}=9.3 \mathrm{~Hz}\right.$, $\mathrm{C}(8) \mathrm{H}), 7.96-7.95(2 \mathrm{H}, \mathrm{m}, \mathrm{C}(3,5) \mathrm{H}), 7.73\left(1 \mathrm{H}, \mathrm{dd},{ }^{3} \mathrm{~J}_{\mathrm{H}-\mathrm{H}}=9.3\right.$, $\left.{ }^{4} J_{H-H}=1.8 \mathrm{~Hz}, \mathrm{C}(7) \mathrm{H}\right), 7.13\left(1 \mathrm{H}, \mathrm{t},{ }^{2} J_{H-F}=56.0 \mathrm{~Hz}, \mathrm{C}\left(4-\mathrm{CHF}_{2}\right) \mathrm{H}\right)$, $6.80\left(1 \mathrm{H}, \mathrm{t},{ }^{2} \mathrm{~J}_{\mathrm{H}-\mathrm{F}}=55.0 \mathrm{~Hz}, \mathrm{C}\left(2-\mathrm{CHF}_{2}\right) \mathrm{H}\right) \mathrm{ppm} .{ }^{19} \mathrm{~F} \delta \mathrm{F}=-57.71(3$ $\left.\mathrm{F}, \mathrm{s}, \mathrm{OCF}_{3}\right),-114.67\left(2 \mathrm{~F}, \mathrm{~d}, 2 \mathrm{~J}_{F-H}=55.0 \mathrm{~Hz}, \mathrm{C} 2-\mathrm{CHF}_{2}\right),-114.88(2$ $\left.\mathrm{F}, \mathrm{d},{ }^{2} J_{F-H}=54.1 \mathrm{~Hz}, \mathrm{C} 4-\mathrm{CHF}_{2}\right) \mathrm{ppm} .{ }^{13} \mathrm{C} \mathrm{NMR} \delta \mathrm{C}=153.23\left(\mathrm{t},{ }^{2} J_{C-F}\right.$ $=27.5 \mathrm{~Hz}, \mathrm{C}-2), 148.97$ (s, C-6), 146.01 (s, C-8-C-N), 140.06 (t, $\left.{ }^{2} J_{C-F}=22.5 \mathrm{~Hz}, \mathrm{C}-4\right), 133.17(\mathrm{~s}, \mathrm{C}-8), 125.23$ (s, C-5-C-C-4), 124.89 (s, C-7), 120.59 (q, $\left.{ }^{1} J_{C-F}=259.2 \mathrm{~Hz}, \mathrm{OCF}_{3}\right), 115.64-$ $115.49(\mathrm{~m}, \mathrm{C}-3), 114.32(\mathrm{~s}, \mathrm{C}-5), 114.08\left(\mathrm{t},{ }^{1} \mathrm{~J}_{\mathrm{C}-F}=241.6 \mathrm{~Hz}\right.$, $\left.\mathrm{C}(2) \mathrm{CHF}_{2}\right), 113.09\left(\mathrm{t},{ }^{1} J_{\mathrm{C}-\mathrm{F}}=240.0 \mathrm{~Hz}, \mathrm{C}(4) \mathrm{CHF}_{2}\right) \mathrm{ppm}$. $\mathrm{C}_{12} \mathrm{H}_{6} \mathrm{~F}_{7} \mathrm{NO}$ (313): calcd (\%) N 4.47, C 45.98, H 1.92, found $\mathrm{N}$ 4.49, C 46.35, H 2.07. MP: $47.5-48.2^{\circ} \mathrm{C}$.

2,4-Bis(difluoromethyl)-N,N-dimethylquinolin-7-amine $3 \mathbf{k}$. The product was prepared according to the general procedure and starting from an activated solution of TFEDMA 4a (1.2 equiv., $0.331 \mathrm{~mL}, 2.83 \mathrm{mmol}$ ) by $\left(\mathrm{BF}_{3} \bullet \mathrm{Et}_{2} \mathrm{O}\right)$ (1.2 equiv., 0.358 $\mathrm{mL}, 2.83 \mathrm{mmol}$ ) and 3-N-(1,1-difluoropropan-2-ylidene)-1- $N, N$ dimethylbenzene-1,3-diamine 1kk (1 equiv., $500 \mathrm{mg}, 2.36$ $\mathrm{mmol}$ ) in presence of anhydrous acetonitrile. 2,4Bis(difluoromethyl)- $N, N$-dimethylquinolin-7-amine $\quad \mathbf{3 k}$ was provided after purification using a gradient of ethyl acetate in pentane (0-5\%) as a brown solid (98.2 $\mathrm{mg}, 15 \%) .{ }^{1} \mathrm{H} \mathrm{NMR} \delta \mathrm{H}=$ $7.94\left(1 \mathrm{H}, \mathrm{d},{ }^{3} \mathrm{~J}_{\mathrm{H}-\mathrm{H}}=9.4 \mathrm{~Hz}, \mathrm{C}(5) \mathrm{H}\right), 7.55(1 \mathrm{H}, \mathrm{s}, \mathrm{C}(8) \mathrm{H}), 7.29(1$ $\left.\mathrm{H}, \mathrm{dd},{ }^{3} J_{H-H}=9.4,{ }^{4} J_{H-H}=2.7 \mathrm{~Hz}, \mathrm{C}(6) \mathrm{H}\right), 7.21(1 \mathrm{H}, \mathrm{d}, J=2.7 \mathrm{~Hz}$, $\mathrm{C}(3) \mathrm{H}), 7.08\left(1 \mathrm{H}, \mathrm{t},{ }^{2} \mathrm{~J}_{\mathrm{H}-\mathrm{F}}=54.6 \mathrm{~Hz}, \mathrm{C}\left(4-\mathrm{CHF}_{2}\right) \mathrm{H}\right), 6.72\left(1 \mathrm{H}, \mathrm{t},{ }^{2} J_{\mathrm{H}-}\right.$ $\left.F=55.4 \mathrm{~Hz}, \mathrm{C}\left(2-\mathrm{CHF}_{2}\right) \mathrm{H}\right), 3.13\left(6 \mathrm{H}, \mathrm{s}, \mathrm{NMe}_{2}\right) \mathrm{ppm} .{ }^{19} \mathrm{~F} \mathrm{NMR} \delta \mathrm{F}=$ $-114.59\left(2 \mathrm{~F}, \mathrm{~d}, 2 \mathrm{~J}_{\mathrm{F}-\mathrm{H}}=54.6 \mathrm{~Hz}, \mathrm{C} 2-\mathrm{CHF}_{2}\right),-114.89\left(2 \mathrm{~F}, \mathrm{~d},{ }^{2} J_{F-H}=\right.$ $\left.55.4 \mathrm{~Hz}, \mathrm{C} 4-\mathrm{CHF}_{2}\right) \mathrm{ppm} .{ }^{13} \mathrm{C} \mathrm{NMR} \delta \mathrm{C}=152.95\left(\mathrm{t},{ }^{2} J_{\mathrm{C}-F}=26.5 \mathrm{~Hz}\right.$, 
C-2), 151.69 (s, C-7), $150.03(\mathrm{~s}, \mathrm{C}-8-\mathrm{C}-\mathrm{N}), 139.30\left(\mathrm{t},{ }^{2} \mathrm{~J}_{\mathrm{C}-\mathrm{F}}=23.9\right.$ Hz, C-4), 124.08 (s, C-5), 118.49 (s, C-8), 116.98 (s, C-5-C-C-4), $114.56\left(\mathrm{t},{ }^{1} J_{C-F}=264.0 \mathrm{~Hz}, \mathrm{C}(2) C H F_{2}\right), 113.54\left(\mathrm{t},{ }^{1} J_{C-F}=240.8 \mathrm{~Hz}\right.$, $\left.\mathrm{C}(4) \mathrm{CHF}_{2}\right), 109.73\left(\mathrm{tt}, 3^{3} \mathrm{~J}_{\mathrm{C}-F}=8.0,3^{3} \mathrm{~J}_{\mathrm{C}-F}=2.2 \mathrm{~Hz}, \mathrm{C}-3\right), 107.26(\mathrm{~s}, \mathrm{C}-$ 6), 40.38 (2 C, s, $\mathrm{NMe}_{2}$ ) ppm. $\mathrm{C}_{13} \mathrm{H}_{12} \mathrm{~F}_{4} \mathrm{~N}_{2}$ (272): calcd (\%) $\mathrm{N}$ 10.20, C 57.30, H 4.41, found N 10.06, C 57.26, H 4.41. MP: $83.7-84.7^{\circ} \mathrm{C}$.

2,4-Bis(difluoromethyl)-N,N-dimethylquinolin-6-amine 3I. The product was prepared according to the general procedure and starting from an activated solution of TFEDMA 4a (1.2 equiv., $0.331 \mathrm{~mL}, 2.83 \mathrm{mmol}$ ) by $\left(\mathrm{BF}_{3} \bullet \mathrm{Et}_{2} \mathrm{O}\right.$ ) (1.2 equiv., $0.358 \mathrm{~mL}, 2.83$ $\mathrm{mmol}$ ) and 4-N-(1,1-difluoropropan-2-ylidene)-1- $N, N$ dimethylbenzene-1,4-diamine 1II (1 equiv., $500 \mathrm{mg}, 2.36$ mmol) in presence of anhydrous acetonitrile. 2,4Bis(difluoromethyl)- $N, N$-dimethylquinolin-6-amine $\quad 3 \mathbf{l}$ was provided after purification using a gradient of ethyl acetate in pentane (0-5\%) as an orange solid (91.9 mg, 14\%). ${ }^{1} \mathrm{H} \mathrm{NMR} \delta \mathrm{H}$ $=8.01\left(1 \mathrm{H}, \mathrm{d},{ }^{3} \mathrm{~J}_{\mathrm{H}-\mathrm{H}}=9.4 \mathrm{~Hz}, \mathrm{C}(8) \mathrm{H}\right), 7.76(1 \mathrm{H}, \mathrm{s}, \mathrm{C}(3) \mathrm{H}), 7.42(1$ $\left.\mathrm{H}, \mathrm{dd},{ }^{3} J_{\mathrm{H}-\mathrm{H}}=9.5,{ }^{4} J_{H-H}=2.7 \mathrm{~Hz}, \mathrm{C}(7) \mathrm{H}\right), 7.07\left(1 \mathrm{H}, \mathrm{t},{ }^{2} J_{H-F}=54.6\right.$ $\left.\mathrm{Hz}, \mathrm{C}\left(4-\mathrm{CHF}_{2}\right) \mathrm{H}\right), 6.90(1 \mathrm{H}, \mathrm{s}, \mathrm{C}(5) \mathrm{H}), 6.75\left(1 \mathrm{H}, \mathrm{t},{ }^{2} J_{\mathrm{H}-\mathrm{F}}=55.5 \mathrm{~Hz}\right.$, $\left.\mathrm{C}\left(2-\mathrm{CHF}_{2}\right) \mathrm{H}\right), 3.14\left(1 \mathrm{H}, \mathrm{s}, \mathrm{NMe}_{2}\right) \mathrm{ppm} .{ }^{19} \mathrm{~F} \mathrm{NMR} \delta \mathrm{F}=-113.38(2$ $\left.\mathrm{F}, \mathrm{d},{ }^{2} \mathrm{~J}_{F-H}=55.5 \mathrm{~Hz}, \mathrm{C} 2-C H F_{2}\right),-116.67\left(2 \mathrm{~F}, \mathrm{~d},{ }^{2} J_{F-H}=54.6 \mathrm{~Hz}\right.$, C4-CHF $F_{2}$ ppm. ${ }^{13} \mathrm{C} N M R \delta C=149.96(s, C-6), 147.42\left(t,{ }^{2} J_{C-F}=\right.$ $26.8 \mathrm{~Hz}, \mathrm{C}-2), 141.73(\mathrm{~s}, \mathrm{C}-8-\mathrm{C}-\mathrm{N}), 136.29\left(\mathrm{t},{ }^{2} \mathrm{~J}_{\mathrm{C}-\mathrm{F}}=21.5 \mathrm{~Hz}, \mathrm{C}-\right.$ 4), 131.36 (s, C-8), 126.89 (s, C-5-C-C-4), 120.03 (s, C-7), 114.90 $\left(\mathrm{t},{ }^{1} J_{C-F}=239.9 \mathrm{~Hz}, \mathrm{C}(2) C H F_{2}\right), 114.64\left(\mathrm{t},{ }^{3} J_{C-F}=8.1 \mathrm{~Hz}, \mathrm{C}-3\right)$, $113.69\left(\mathrm{t},{ }^{1} J_{C-F}=241.4 \mathrm{~Hz}, \mathrm{C}(4) C H F_{2}\right), 99.55(\mathrm{~s}, \mathrm{C}-5), 40.53$ (s, $\mathrm{NMe}_{2}$ ) ppm. $\mathrm{C}_{13} \mathrm{H}_{12} \mathrm{~F}_{4} \mathrm{~N}_{2}$ (272): calcd (\%) N 10.20, C 57.30, H 4.41, found N 10.09, C 56.86, H 4.40. MP: $115.6-116.9^{\circ} \mathrm{C}$.

2,4-Bis(difluoromethyl)-7-fluoro-8-methylquinoline $3 \mathrm{~m}$. The product was prepared according to the general procedure and starting from an activated solution of TFEDMA 4a (1.2 equiv., $0.698 \mathrm{~mL}, 5.96 \mathrm{mmol}$ ) by $\left(\mathrm{BF}_{3} \bullet \mathrm{Et}_{2} \mathrm{O}\right)$ (1.2 equiv., $0.756 \mathrm{~mL}, 5.96$ $\mathrm{mmol}$ ) and $\mathrm{N}$-(1,1-difluoropropan-2-ylidene)-3-fluoro-2methylaniline $1 \mathrm{~mm}$ ( 1 equiv., $1 \mathrm{~g}, 4.97 \mathrm{mmol}$ ) in presence of anhydrous acetonitrile. 2,4-Bis(difluoromethyl)-7-fluoro-8methylquinoline $3 \mathrm{~m}$ was provided after purification using a gradient of ethyl acetate in pentane (0-5\%) as an yellow solid (990 mg, 76\%). ${ }^{1} \mathrm{H} \mathrm{NMR} \delta \mathrm{H}=7.96\left(1 \mathrm{H}, \mathrm{dd},{ }^{3} J_{H-H}=9.0,{ }^{4} J_{H-F}=\right.$ $5.9 \mathrm{~Hz}, \mathrm{C}(5) \mathrm{H}), 7.86(1 \mathrm{H}, \mathrm{s}, \mathrm{C}(3) \mathrm{H}), 7.47\left(1 \mathrm{H}, \mathrm{t},{ }^{3} J_{H-H}=9.0 \mathrm{~Hz}\right.$, $\mathrm{C}(6) \mathrm{H}), 7.13\left(1 \mathrm{H}, \mathrm{t},{ }^{2} \mathrm{~J}_{\mathrm{H}-\mathrm{F}}=54.3 \mathrm{~Hz}, \mathrm{C}\left(4-\mathrm{CHF}_{2}\right) \mathrm{H}\right), 6.81\left(1 \mathrm{H}, \mathrm{t},{ }^{2} \mathrm{~J}_{\mathrm{H}}\right.$ $\left.F=55.1 \mathrm{~Hz}, \mathrm{C}\left(2-\mathrm{CHF}_{2}\right) \mathrm{H}\right), 2.71\left(3 \mathrm{H}, \mathrm{d},{ }^{4} J_{H-F}=2.5 \mathrm{~Hz}, \mathrm{Me}\right) \mathrm{ppm}$. ${ }^{19} \mathrm{~F} N M R \delta F=-109.41\left(1 \mathrm{~F}, \mathrm{ddd},{ }^{3} J_{H-F}=8.4,{ }^{4} J_{H-F}=5.6,{ }^{4} J_{C H 3-F}=\right.$ $2.7 \mathrm{~Hz}, \mathrm{~F},),-114.20\left(2 \mathrm{~F}, \mathrm{~d},{ }^{2} J_{H-F}=55.1 \mathrm{~Hz}, \mathrm{C} 2-\mathrm{CHF}_{2}\right),-114.56(2$ $\left.\mathrm{F}, \mathrm{d},{ }^{2} J_{\mathrm{H}-\mathrm{F}}=54.3 \mathrm{~Hz}, \mathrm{C} 4-\mathrm{CHF}_{2}\right) \mathrm{ppm} .{ }^{13} \mathrm{C} \mathrm{NMR} \delta \mathrm{C}=161.37\left(\mathrm{~d},{ }^{1} J_{\mathrm{C}-F}\right.$ $=249.1 \mathrm{~Hz}, \mathrm{C}-7), 152.53\left(\mathrm{t},{ }^{2} J_{C-F}=27.5 \mathrm{~Hz}, \mathrm{C}-2\right), 148.08\left(\mathrm{~d},{ }^{3} J_{C-F}=\right.$ $10.1 \mathrm{~Hz}, \mathrm{C}-8-C-\mathrm{N}), 140.19\left(\mathrm{t},{ }^{2} J_{C-F}=21.5 \mathrm{~Hz}, \mathrm{C}-4\right), 123.23\left(\mathrm{~d},{ }^{2} J_{C-F}\right.$ $=16.1 \mathrm{~Hz}, \mathrm{C}-8$ ), 122.36 (d, $\left.{ }^{3} J_{C-F}=10.2 \mathrm{~Hz}, \mathrm{C}-5\right), 121.85$ (s, C-5-CC-4), $119.38\left(\mathrm{~d},{ }^{2} J_{C-F}=27.0 \mathrm{~Hz}, \mathrm{C}-6\right), 114.58\left(\mathrm{t},{ }^{1} J_{C-F}=241.2 \mathrm{~Hz}\right.$, $\left.\mathrm{C}(2) \mathrm{CHF}_{2}\right), 113.12\left(\mathrm{t},{ }^{1} J_{C-F}=242.4 \mathrm{~Hz}, \mathrm{C}(4) C H F_{2}\right), 113.12(\mathrm{~s}, \mathrm{C}-3)$, $9.34\left(d,{ }^{4} J_{H-F}=3.8 \mathrm{~Hz}, \mathrm{Me}\right)$ ppm. $\mathrm{C}_{12} \mathrm{H}_{8} \mathrm{~F}_{5} \mathrm{~N}$ (261): calcd (\%) $\mathrm{N}$ 5.36, C 55.13, H 3.06, found N 5.26, C 55.13, H 3.22. MP: 87.7 $89^{\circ} \mathrm{C}$.

7-Chloro-2,4-bis(difluoromethyl)-8-methylquinoline $3 \mathrm{n}$. The product was prepared according to the general procedure and starting from an activated solution of TFEDMA 4a (1.2 equiv., $0.662 \mathrm{~mL}, 5.66 \mathrm{mmol}$ ) by $\left(\mathrm{BF}_{3} \bullet \mathrm{Et}_{2} \mathrm{O}\right)$ (1.2 equiv., $0.717 \mathrm{~mL}, 5.66$ $\mathrm{mmol}$ and 3-chloro-N-(1,1-difluoropropan-2-ylidene)-2methylaniline $1 \mathrm{nn}$ (1 equiv., $1.03 \mathrm{~g}, 4.72 \mathrm{mmol}$ ) in presence of anhydrous acetonitrile. 7-Chloro-2,4-bis(difluoromethyl)-8methylquinoline $\mathbf{3 n}$ was provided after purification using a gradient of ethyl acetate in pentane $(0-5 \%)$ as a colourless solid $(977 \mathrm{mg}, 75 \%) .{ }^{1} \mathrm{H} \mathrm{NMR} \delta \mathrm{H}=7.89-7.88(2 \mathrm{H}, \mathrm{m}$, $\mathrm{C}(3,5) \mathrm{H}), 7.67\left(1 \mathrm{H}, \mathrm{d},{ }^{3} J_{\mathrm{H}-\mathrm{H}}=9.1 \mathrm{~Hz}, \mathrm{C}(6) \mathrm{H}\right), 7.13\left(1 \mathrm{H}, \mathrm{t},{ }^{2} J_{\mathrm{H}-\mathrm{F}}=\right.$ $\left.54.3 \mathrm{~Hz}, \mathrm{C}\left(4-\mathrm{CHF}_{2}\right) \mathrm{H}\right), 6.80\left(1 \mathrm{H}, \mathrm{t},{ }^{2} \mathrm{~J}_{\mathrm{H}-\mathrm{F}}=55.1 \mathrm{~Hz}, \mathrm{C}\left(2-\mathrm{CHF}_{2}\right) \mathrm{H}\right)$, $2.88(3 \mathrm{H}, \mathrm{s}, \mathrm{Me}) \mathrm{ppm} .{ }^{19} \mathrm{~F} N M R \delta \mathrm{F}=-114.17\left(2 \mathrm{~F}, \mathrm{~d},{ }^{2} \mathrm{~J}_{F-H}=55.1\right.$ $\left.\mathrm{Hz}, \mathrm{C} 2-\mathrm{CHF}_{2}\right),-114.75\left(2 \mathrm{~F}, \mathrm{~d},{ }^{2} \mathrm{~J}_{F-H}=54.3 \mathrm{~Hz}, \mathrm{C} 4-\mathrm{CHF}_{2}\right) \mathrm{ppm} .{ }^{13} \mathrm{C}$ NMR $\delta C=152.34\left(\mathrm{t},{ }^{2} J_{C-F}=27.6 \mathrm{~Hz}, \mathrm{C}-2\right), 147.27(\mathrm{~s}, \mathrm{C}-8-\mathrm{C}-\mathrm{N})$, $140.27\left(\mathrm{t},{ }^{2} J_{C-F}=22.2 \mathrm{~Hz}, \mathrm{C}-4\right), 136.83(\mathrm{~s}, \mathrm{C}-7), 136.57(\mathrm{~s}, \mathrm{C}-8)$, 130.56 (s, C-6), 123.48 (s, C-5-C-C-4), 121.65 (s, C-5), 114.52 (t, $\left.{ }^{1} J_{C-F}=241.3 \mathrm{~Hz}, \mathrm{C}(2) C H F_{2}\right), 113.97\left(\mathrm{t},{ }^{3} J_{C-F}=7.9 \mathrm{~Hz}, \mathrm{C}-3\right), 113.08$ $\left(\mathrm{t},{ }^{1} J_{\mathrm{C}-\mathrm{F}}=242.4 \mathrm{~Hz}, \mathrm{C}(4) \mathrm{CHF}_{2}\right), 14.84$ (s, Me) ppm. $\mathrm{C}_{12} \mathrm{H}_{8} \mathrm{~F}_{4} \mathrm{NCl}$ (277): calcd (\%) N 5.04, C 51.86, H 2.88, found N 4.92, C 52.02, H 3.03. MP: $58.9-59.5^{\circ} \mathrm{C}$.

7-Chloro-2,4-bis(difluoromethyl)quinoline 30. The product was prepared according to the general procedure and starting from an activated solution of TFEDMA 4a (1.2 equiv., $3.45 \mathrm{~mL}$, $29.5 \mathrm{mmol}$ ) by $\left(\mathrm{BF}_{3} \bullet \mathrm{Et}_{2} \mathrm{O}\right)$ (1.2 equiv., $\left.3.73 \mathrm{~mL}, 29.5 \mathrm{mmol}\right)$ and 3-chloro- $N$-(1,1-difluoropropan-2-ylidene)aniline 100 (1 equiv., $5 \mathrm{~g}, 24.6 \mathrm{mmol}$ ) in presence of anhydrous acetonitrile. 7Chloro-2,4-bis(difluoromethyl)quinoline 3o was provided after purification using a gradient of ethyl acetate in pentane (0-5\%) as a colourless solid $(2.93 \mathrm{~g}, 45 \%) .{ }^{1} \mathrm{H} N M R \delta \mathrm{H}=8.20(1 \mathrm{H}, \mathrm{d}$, $\left.{ }^{4} J_{H-H}=2.1 \mathrm{~Hz}, \mathrm{C}(8) \mathrm{H}\right), 8.07\left(1 \mathrm{H}, \mathrm{d},{ }^{3} J_{H-H}=9.0 \mathrm{~Hz}, \mathrm{C}(5) \mathrm{H}\right), 7.88(1$ $\mathrm{H}, \mathrm{s}, \mathrm{C}(3) \mathrm{H}), 7.67\left(1 \mathrm{H}, \mathrm{dd},{ }^{3} \mathrm{~J}_{\mathrm{H}-\mathrm{H}}=9.0,{ }^{4} \mathrm{~J}_{\mathrm{H}-\mathrm{H}}=2.1 \mathrm{~Hz}, \mathrm{C}(6) \mathrm{H}\right), 7.12$ $\left(1 \mathrm{H}, \mathrm{t},{ }^{2} J_{\mathrm{H}-\mathrm{F}}=54.2 \mathrm{~Hz}, \mathrm{C}\left(4-\mathrm{CHF}_{2}\right) \mathrm{H}\right), 6.77\left(1 \mathrm{H}, \mathrm{t},{ }^{2} J_{\mathrm{H}-\mathrm{F}}=55.0 \mathrm{~Hz}\right.$, $\left.\mathrm{C}\left(2-\mathrm{CHF}_{2}\right) \mathrm{H}\right) \mathrm{ppm} .{ }^{19} \mathrm{~F} N M R \delta \mathrm{F}=-114.73\left(4 \mathrm{~F}, \mathrm{t},{ }^{2} J_{F-H}=54.4 \mathrm{~Hz}\right.$, $\left.\mathrm{C} 2-\mathrm{CHF}_{2} / \mathrm{C}-\mathrm{CHF}_{2}\right) \mathrm{ppm} .{ }^{13} \mathrm{C} \mathrm{NMR} \delta \mathrm{C}=153.83\left(\mathrm{t},{ }^{2} \mathrm{~J}_{\mathrm{C}-\mathrm{F}}=27.3 \mathrm{~Hz}\right.$, C-2), $148.27(\mathrm{~s}, \mathrm{C} 8-\mathrm{C}-\mathrm{N}), 140.12\left(\mathrm{t},{ }^{2} \mathrm{~J}_{\mathrm{C}-\mathrm{F}}=22.4 \mathrm{~Hz}, \mathrm{C}-4\right), 137.12$ (s, C-7), 130.33 (s, C-6), 129.71 (s, C-8), 124.95 (s, C-5), 123.13 (s, C-5-C-C-4), $114.58\left(\mathrm{tt},{ }^{3} \mathrm{~J}_{C-F}=8,{ }^{3} \mathrm{~J}_{\mathrm{C}-F}=1.8 \mathrm{~Hz}, \mathrm{C}-3\right), 114.09(\mathrm{t}$, $\left.{ }^{1} J_{C-F}=242.9 \mathrm{~Hz}, \mathrm{C}(2) C H F_{2}\right), 113.08\left(\mathrm{t},{ }^{1} J_{C-F}=241.5 \mathrm{~Hz}, \mathrm{C}(4) C H F_{2}\right)$ ppm. $\mathrm{C}_{11} \mathrm{H}_{6} \mathrm{~F}_{4} \mathrm{NCl}$ (263): calcd (\%) N 5.31, C 50.12, H 2.29, found N 5.23, C 50.00, H 2.29. MP: $90.2-91^{\circ} \mathrm{C}$.

2,4-Bis(difluoromethyl)-6-(trifluoromethyl)quinoline 3p. The product was prepared according to the general procedure and starting from an activated solution of TFEDMA 4a (1.2 equiv., $0.195 \mathrm{~mL}, 1.66 \mathrm{mmol})$ by $\left(\mathrm{BF}_{3} \bullet \mathrm{Et}_{2} \mathrm{O}\right)$ (1.2 equiv., $0.211 \mathrm{~mL}, 1.66$ $\mathrm{mmol}$ and $\mathrm{N}$-(1,1-difluoropropan-2-ylidene)-4(trifluoromethyl)aniline 1pp (1 equiv., $328 \mathrm{mg}, 1.39 \mathrm{mmol}$ ) in presence of anhydrous acetonitrile. 2,4-Bis(difluoromethyl)-6(trifluoromethyl)quinoline $\mathbf{3 p}$ was provided after purification using a gradient of ethyl acetate in pentane $(0-5 \%)$ as a grey solid (93.9 mg, 23\%). ${ }^{1} \mathrm{H}$ NMR $\delta \mathrm{H}=8.46(1 \mathrm{H}, \mathrm{s}, \mathrm{C}(5) \mathrm{H}), 8.37(1$ $\left.\mathrm{H}, \mathrm{d},{ }^{3} J_{H-H}=8.9 \mathrm{~Hz}, \mathrm{C}(8) \mathrm{H}\right), 8.04\left(1 \mathrm{H}, \mathrm{dd},{ }^{3} J_{H-H}=8.9,{ }^{4} J_{H-H}=1.8\right.$ $\mathrm{Hz}, \mathrm{C}(7) \mathrm{H}), 8.00(1 \mathrm{H}, \mathrm{s}, \mathrm{C}(3) \mathrm{H}), 7.20\left(1 \mathrm{H}, \mathrm{t},{ }^{2} \mathrm{~J}_{\mathrm{H}-\mathrm{F}}=54.0 \mathrm{~Hz}, \mathrm{C}(4-\right.$ $\left.\left.\mathrm{CHF}_{2}\right) \mathrm{H}\right), 6.82\left(1 \mathrm{H}, \mathrm{t},{ }^{2} J_{\mathrm{H}-\mathrm{F}}=54.9 \mathrm{~Hz}, \mathrm{C}\left(2-\mathrm{CHF}_{2}\right) \mathrm{H}\right) \mathrm{ppm} .{ }^{19} \mathrm{~F} \mathrm{NMR}$ $\delta \mathrm{F}=-62.70\left(3 \mathrm{~F}, \mathrm{~s}, \mathrm{CF}_{3}\right),-114.48\left(2 \mathrm{~F}, \mathrm{~d}, 2 \mathrm{~J}_{\mathrm{F}-\mathrm{H}}=54.0 \mathrm{~Hz}, \mathrm{C} 2-\right.$ $\left.\mathrm{CHF}_{2}\right),-114.98\left(2 \mathrm{~F}, \mathrm{~d},{ }^{2} \mathrm{~J}_{F-H}=54.9 \mathrm{~Hz}, \mathrm{C} 4-\mathrm{CHF}_{2}\right) \mathrm{ppm} .{ }^{13} \mathrm{C} \mathrm{NMR}$ $\delta \mathrm{C}=154.90\left(\mathrm{t},{ }^{2} J_{C-F}=27.4 \mathrm{~Hz}, \mathrm{C}-2\right), 148.77(\mathrm{~s}, \mathrm{C}-8-\mathrm{C}-\mathrm{N}), 141.09$ $\left(\mathrm{t},{ }^{2} J_{C-F}=22.5 \mathrm{~Hz}, \mathrm{C}-4\right), 132.14(\mathrm{~s}, \mathrm{C}-8), 131.11\left(\mathrm{q},{ }^{2} J_{C-F}=33.1 \mathrm{~Hz}\right.$, C-6), $126.76\left(\mathrm{q},{ }^{3} J_{C-F}=2.9 \mathrm{~Hz}, \mathrm{C}-7\right), 123.95\left(\mathrm{t},{ }^{3} J_{C-F}=2.5 \mathrm{~Hz}, \mathrm{C}-5-\right.$ $C-C-4), 123.65\left(q,{ }^{1} J_{C-F}=272.9 \mathrm{~Hz}, \mathrm{CF}_{3}\right), 121.81(\mathrm{~s}, \mathrm{C}-5), 115.71$ $\left(\mathrm{tt},{ }^{3} J_{C-F}=7,6,{ }^{3} J_{C-F}=2,2 \mathrm{~Hz}, \mathrm{C}-3\right), 113.94\left(\mathrm{t},{ }^{1} J_{C-F}=243.4 \mathrm{~Hz}\right.$, 
$\left.\mathrm{C}(2) \mathrm{CHF}_{2}\right), 112.93\left(\mathrm{t},{ }^{1} J_{\mathrm{C}-\mathrm{F}}=241.9 \mathrm{~Hz}, \mathrm{C}(4) \mathrm{CHF}_{2}\right) \mathrm{ppm} . \mathrm{C}_{12} \mathrm{H}_{6} \mathrm{~F}_{7} \mathrm{~N}$ (297): calcd (\%) N 4.71, C 48.50, H 2.04, found N 4.78, C 48.51, H 2.17. MP: $57.7-58.3^{\circ} \mathrm{C}$.

2,4-Bis(difluoromethyl)-7-(trifluoromethyl)quinoline 3q. The product was prepared according to the general procedure and starting from an activated solution of TFEDMA 4 a (1.2 equiv., $0.56 \mathrm{~mL}, 4.79 \mathrm{mmol}$ ) by $\left(\mathrm{BF}_{3} \bullet \mathrm{Et}_{2} \mathrm{O}\right)$ (1.2 equiv., $0.606 \mathrm{~mL}, 4.79$ $\mathrm{mmol}$ and $\mathrm{N}$-(1,1-difluoropropan-2-ylidene)-3(trifluoromethyl)aniline 1qq (1 equiv., $0.946 \mathrm{~g}, 3.99 \mathrm{mmol}$ ) in presence of anhydrous acetonitrile. 2,4-Bis(difluoromethyl)-7(trifluoromethyl)quinoline $\mathbf{3 q}$ was provided after purification using a gradient of ethyl acetate in pentane (0-5\%) as a colourless solid $(328.5 \mathrm{mg}, 28 \%) .{ }^{1} \mathrm{H} N M R \delta \mathrm{H}=8.56(1 \mathrm{H}, \mathrm{s}$, $\mathrm{C}(8) \mathrm{H}), 8.31\left(1 \mathrm{H}, \mathrm{d},{ }^{3} J_{\mathrm{H}-\mathrm{H}}=8.9 \mathrm{~Hz}, \mathrm{C}(5) \mathrm{H}\right), 8.02(1 \mathrm{H}, \mathrm{s}, \mathrm{C}(3) \mathrm{H})$, $7.93\left(1 \mathrm{H}, \mathrm{d}, 3 J_{H-H}=8.7 \mathrm{~Hz}, \mathrm{C}(6) \mathrm{H}\right), 7.19\left(1 \mathrm{H}, \mathrm{t},{ }^{2} J_{H-F}=54.1 \mathrm{~Hz}\right.$, $\left.\mathrm{C}\left(4-\mathrm{CHF}_{2}\right) \mathrm{H}\right), 6.82\left(1 \mathrm{H}, \mathrm{t},{ }^{2} J_{\mathrm{H}-\mathrm{F}}=54.9 \mathrm{~Hz}, \mathrm{C}\left(2-\mathrm{CHF}_{2}\right) \mathrm{H}\right) \mathrm{ppm} .{ }^{19} \mathrm{~F}$ NMR $\delta F=-63.10\left(3 \mathrm{~F}, \mathrm{~s}, \mathrm{CF}_{3}\right),-114.71\left(2 \mathrm{~F}, \mathrm{~d},{ }^{2} J_{\mathrm{F}-\mathrm{H}}=56.4 \mathrm{~Hz}, \mathrm{C} 2-\right.$ $\mathrm{CHF}_{2}$ ), $-114.81\left(2 \mathrm{~F}, \mathrm{~d},{ }^{2} J_{F-H}=54.8 \mathrm{~Hz}, \mathrm{C} 4-\mathrm{CHF}_{2}\right) \mathrm{ppm} .{ }^{13} \mathrm{C} \mathrm{NMR}$ $\delta \mathrm{C}=154.19\left(\mathrm{t},{ }^{2} J_{\mathrm{C}-F}=27.5 \mathrm{~Hz}, \mathrm{C}-2\right), 146.86(\mathrm{~s}, \mathrm{C}-8-\mathrm{C}-\mathrm{N}), 140.20$ $\left(\mathrm{t},{ }^{2} J_{C-F}=22.6 \mathrm{~Hz}, \mathrm{C}-4\right), 132.69\left(\mathrm{q},{ }^{2} J_{C-F}=33.7 \mathrm{~Hz}, \mathrm{C}-7\right), 128.43(\mathrm{~s}$, C-8), 126.15 (s, C-5-C-C-4), 125.08 (s, C-5), 124.85 (s, C-6), $123.54\left(\mathrm{q},{ }^{1} J_{C-F}=272.4 \mathrm{~Hz}, \mathrm{CF}_{3}\right), 116.20\left(\mathrm{t},{ }^{3} J_{C-F}=7.6 \mathrm{~Hz}, \mathrm{C}-3\right)$, $114.02\left(\mathrm{t},{ }^{1} J_{C-F}=242.3 \mathrm{~Hz}, \mathrm{C}(2) C H F_{2}\right), 112.98\left(\mathrm{t},{ }^{1} J_{C-F}=241.5 \mathrm{~Hz}\right.$, $\mathrm{C}(4) \mathrm{CHF}_{2}$ ) ppm. $\mathrm{C}_{12} \mathrm{H}_{6} \mathrm{~F}_{7} \mathrm{~N}$ (297): calcd (\%) N 4.71, C 48.50, $\mathrm{H}$ 2.04, found N 4.74, C 48.35, H 2.10. MP: $39.7-40^{\circ} \mathrm{C}$.

2,4-Bis(difluoromethyl)-8-(trifluoromethyl)quinoline $3 r$. The product was prepared according to the general procedure and starting from an activated solution of TFEDMA 4a (1.2 equiv., $0.178 \mathrm{~mL}, 1.52 \mathrm{mmol})$ by $\left(\mathrm{BF}_{3} \bullet \mathrm{Et}_{2} \mathrm{O}\right)(1.2$ equiv., $0.192 \mathrm{~mL}, 1.52$ $\mathrm{mmol}$ and N-(1,1-Difluoropropan-2-ylidene)-2(trifluoromethyl)aniline 1rr (1 equiv., $0.5 \mathrm{~g}, 1.26 \mathrm{mmol}$ ) in presence of anhydrous acetonitrile. 2,4-Bis(difluoromethyl)-8(trifluoromethyl)quinoline $3 r$ was provided after purification using a gradient of ethyl acetate in pentane $(0-5 \%)$ as a colourless solid (traces). ${ }^{1} \mathrm{H} N M R \delta \mathrm{H}=8.37\left(1 \mathrm{H}, \mathrm{d},{ }^{2} J_{\mathrm{H}-\mathrm{H}}=8.4\right.$ $\mathrm{Hz}, \mathrm{C}(7) \mathrm{H}), 8.22\left(1 \mathrm{H}, \mathrm{d},{ }^{2} J_{H-H}=7.3 \mathrm{~Hz}, \mathrm{C}(5) \mathrm{H}\right), 8.02(1 \mathrm{H}, \mathrm{s}$, $\mathrm{C}(3) \mathrm{H}), 7.81\left(1 \mathrm{H}, \mathrm{t},{ }^{3} \mathrm{~J}_{\mathrm{H}-\mathrm{H}}=7.9 \mathrm{~Hz}, \mathrm{C}(6) \mathrm{H}\right), 7.18\left(1 \mathrm{H}, \mathrm{t},{ }^{2} \mathrm{~J}_{\mathrm{H}-\mathrm{F}}=\right.$ $\left.54.1 \mathrm{~Hz}, \mathrm{C}\left(4-\mathrm{CHF}_{2}\right) \mathrm{H}\right), 6.84\left(1 \mathrm{H}, \mathrm{t},{ }^{2} J_{\mathrm{H}-\mathrm{F}}=54.9 \mathrm{~Hz}, \mathrm{C}\left(2-\mathrm{CHF}_{2}\right) \mathrm{H}\right)$. ${ }^{19} \mathrm{~F} \mathrm{NMR} \delta \mathrm{F}=-60.17\left(3 \mathrm{~F}, \mathrm{~s}, \mathrm{CF}_{3}\right),-114.25\left(2 \mathrm{~F}, \mathrm{~d},{ }^{2} \mathrm{~J}_{\mathrm{F}-\mathrm{H}}=56.4 \mathrm{~Hz}\right.$, $\left.\mathrm{C} 2-\mathrm{CHF}_{2}\right),-114.36\left(2 \mathrm{~F}, \mathrm{~d},{ }^{2} J_{F-H}=54.8 \mathrm{~Hz}, \mathrm{C} 4-\mathrm{CHF}_{2}\right) \mathrm{ppm}$.

2,4-Bis(difluoromethyl)-8-phenylquinoline 3s. The product was prepared according to the general procedure and starting from an activated solution of TFEDMA 4a (1.2 equiv., $0.403 \mathrm{~mL}$, $3.45 \mathrm{mmol}$ ) by $\left(\mathrm{BF}_{3} \bullet \mathrm{Et}_{2} \mathrm{O}\right)$ (1.2 equiv., $0.437 \mathrm{~mL}, 3.45 \mathrm{mmol}$ ) and $\mathrm{N}$-(1,1-difluoropropan-2-ylidene)-2-phenylaniline 1ss (1 equiv., $704 \mathrm{mg}, 2.87 \mathrm{mmol}$ ) in presence of anhydrous acetonitrile. 2,4-Bis(difluoromethyl)-8-phenylquinoline 3 s was provided after purification using a gradient of ethyl acetate in pentane (0-5\%) as a colour less solid (248.9 mg, 28\%). ${ }^{1} \mathrm{H}$ NMR $\delta \mathrm{H}=8.16\left(1 \mathrm{H}, \mathrm{dd},{ }^{3} \mathrm{~J}_{\mathrm{H}-\mathrm{H}}=8.5,{ }^{4} \mathrm{~J}_{\mathrm{H}-\mathrm{H}}=1.5 \mathrm{~Hz}, \mathrm{C}(5) \mathrm{H}\right), 8.00(1 \mathrm{H}, \mathrm{s}$, $\mathrm{C}(3) \mathrm{H}), 7.92\left(1 \mathrm{H}, \mathrm{dd}, 3_{\mathrm{H}-\mathrm{H}}=7.2,{ }^{4} \mathrm{~J}_{\mathrm{H}-\mathrm{H}}=1.3 \mathrm{~Hz}, \mathrm{C}(7) \mathrm{H}\right), 7.85-$ $7.77(1 \mathrm{H}, \mathrm{m}, \mathrm{C}(6) \mathrm{H}), 7.76-7.70\left(2 \mathrm{H}, \mathrm{m}, \mathrm{C}\left(5^{\prime}\right) \mathrm{H}\right), 7.58-7.45(3$ $\left.\mathrm{H}, \mathrm{m}, \mathrm{C}\left(6^{\prime}\right) \mathrm{H}\right), 7.24\left(1 \mathrm{H}, \mathrm{t},{ }^{2} J_{\mathrm{H}-\mathrm{F}}=54.3 \mathrm{~Hz}, \mathrm{C}\left(4-\mathrm{CHF}_{2}\right) \mathrm{H}\right), 6.74(1$ $\left.\mathrm{H}, \mathrm{t},{ }^{2} J_{\mathrm{H}-\mathrm{F}}=55.1 \mathrm{~Hz}, \mathrm{C}\left(2-\mathrm{CHF}_{2}\right) \mathrm{H}\right) \mathrm{ppm} .{ }^{19} \mathrm{~F} \mathrm{NMR} \delta \mathrm{F}=-113.70(2$ $\left.\mathrm{F}, \mathrm{d},{ }^{2} \mathrm{~J}_{F-H}=55.1 \mathrm{~Hz}, \mathrm{C} 2-C H F_{2}\right),-114.97\left(2 \mathrm{~F}, \mathrm{~d},{ }^{2} J_{F-H}=54.3 \mathrm{~Hz}\right.$, C4-CHF $F_{2}$ ppm. ${ }^{13} \mathrm{C} \mathrm{NMR} \delta \mathrm{C}=152.18\left(\mathrm{t},{ }^{2} J_{\mathrm{C}-F}=27.8 \mathrm{~Hz}, \mathrm{C}-2\right)$, $145.30(\mathrm{~s}, \mathrm{C}-8-\mathrm{C}-\mathrm{N}), 142.28\left(\mathrm{~s}, \mathrm{C}-1^{\prime}\right), 140.04\left(\mathrm{t},{ }^{2} \mathrm{~J}_{\mathrm{C}-F}=22.0 \mathrm{~Hz}, \mathrm{C}-\right.$
4), 138.65 (s, C-8), 131.61 (s, C-7), 131.02 (2 C, s, C-3', $\left.5^{\prime}\right)$, 129.09 (s, C-6), 128.06 (2 C, s, C-2', 6'), 127.84 (s, C-4'), 125.29 (s, C-5-C-C-4), 122.83 (s, C-5), $114.68\left(\mathrm{t},{ }^{1} J_{C-F}=240.9 \mathrm{~Hz}\right.$, $\left.\mathrm{C}(2) \mathrm{CHF}_{2}\right), 114.17\left(\mathrm{t},{ }^{3} \mathrm{~J}_{\mathrm{C}-\mathrm{F}}=8.0 \mathrm{~Hz}, \mathrm{C}-3\right), 113.25\left(\mathrm{t},{ }^{1} \mathrm{~J}_{\mathrm{C}-\mathrm{F}}=242.4\right.$ $\mathrm{Hz}, \mathrm{C}(4) \mathrm{CHF}_{2}$ ) ppm. $\mathrm{C}_{1} \mathrm{H}_{11} \mathrm{~F}_{4} \mathrm{~N}$ (305): calcd (\%) N 4.59, C 66.89, H 3.63, found N 4.71, C 66.99, H 3.70. MP: $58.6-59.5^{\circ} \mathrm{C}$.

2,4-Bis(difluoromethyl)-8-(phenylsulfanyl)quinoline $3 \mathrm{t}$. The product was prepared according to the general procedure and starting from an activated solution of TFEDMA 4a (1.2 equiv., $0.253 \mathrm{~mL}, 2.16 \mathrm{mmol})$ by $\left(\mathrm{BF}_{3} \bullet \mathrm{Et}_{2} \mathrm{O}\right)$ (1.2 equiv., $0.274 \mathrm{~mL}, 2.16$ $\mathrm{mmol}$ and $\quad \mathrm{N}$-(1,1-difluoropropan-2-ylidene)-2(phenylsulfanyl)aniline 1tt (1 equiv., $500 \mathrm{mg}, 1.8 \mathrm{mmol}$ ) in presence of anhydrous acetonitrile. 2,4-Bis(difluoromethyl)-8(phenylsulfanyl)quinoline 3t was provided after purification using a gradient of ethyl acetate in pentane (0-5\%) as a colour less solid (308.7 mg, 52\%). ${ }^{1} \mathrm{H}$ NMR $\delta \mathrm{H}=7.99(1 \mathrm{H}, \mathrm{s}, \mathrm{C}(3) \mathrm{H})$, $7.82\left(1 \mathrm{H}, \mathrm{d},{ }^{3} \mathrm{~J}_{\mathrm{H}-\mathrm{H}}=8.4 \mathrm{~Hz}, \mathrm{C}(5) \mathrm{H}\right), 7.66\left(2 \mathrm{H}, \mathrm{dd},{ }^{3} J_{H-H}=6.4,{ }^{4} J_{H-H}\right.$ $\left.=3.0 \mathrm{~Hz}, \mathrm{C}\left(2^{\prime}, 6^{\prime}\right) \mathrm{H}\right), 7.53-7.49\left(3 \mathrm{H}, \mathrm{m}, \mathrm{C}\left(3^{\prime}, 4^{\prime}, 5^{\prime}\right) \mathrm{H},\right), 7.47(1 \mathrm{H}$, $\left.\mathrm{t},{ }^{3} J_{\mathrm{H}-\mathrm{H}}=8.0 \mathrm{~Hz}, \mathrm{C}(6) \mathrm{H}\right), 7.17\left(1 \mathrm{H}, \mathrm{t},{ }^{2} J_{\mathrm{H}-\mathrm{F}}=54.3 \mathrm{~Hz}, \mathrm{C}\left(4-\mathrm{CHF}_{2}\right) \mathrm{H}\right)$, $7.06\left(1 \mathrm{H}, \mathrm{d},{ }^{3} J_{\mathrm{H}-\mathrm{H}}=7.6 \mathrm{~Hz}, \mathrm{C}(7) \mathrm{H}\right), 6.89\left(1 \mathrm{H}, \mathrm{t},{ }^{2} \mathrm{~J}_{\mathrm{H}-\mathrm{F}}=54.9 \mathrm{~Hz}\right.$, $\left.\mathrm{C}\left(2-\mathrm{CHF}_{2}\right) \mathrm{H}\right) \mathrm{ppm} .{ }^{19} \mathrm{~F} \mathrm{NMR} \delta \mathrm{F}=-113.66\left(2 \mathrm{~F}, \mathrm{~d},{ }^{2} J_{F-H}=55.0 \mathrm{~Hz}\right.$, $\left.\mathrm{C} 2-\mathrm{CHF}_{2}\right),-115.22\left(2 \mathrm{~F}, \mathrm{~d},{ }^{2} \mathrm{~J}_{F-H}=54.2 \mathrm{~Hz}, \mathrm{C} 4-\mathrm{CHF}_{2}\right) \mathrm{ppm} .{ }^{13} \mathrm{C}$ NMR $\delta C=151.47\left(t,{ }^{2} J_{C-F}=27.7 \mathrm{~Hz}, \mathrm{C}-2\right), 143.85(\mathrm{~s}, \mathrm{C}-8-C-\mathrm{N})$, $143.02(\mathrm{~s}, \mathrm{C}-8), 140.20\left(\mathrm{t},{ }^{2} J_{\mathrm{C}-F}=22.2 \mathrm{~Hz}, \mathrm{C}-4\right), 136.16$ (2 C, s, C$\left.2^{\prime}, 6^{\prime}\right), 130.89$ (s, C-1'), 130.11 (2 C, s, C-3', 5'), 129.64 (s, C-4'), 129.33 (s, C-6), 126.16 (s, C-7), 125.13 (s, C-5-C-C-4), 119.37 (s, C-5), $114.86\left(\mathrm{t},{ }^{3} J_{C-F}=7.9 \mathrm{~Hz}, \mathrm{C}-3\right), 114.31\left(\mathrm{t},{ }^{1} J_{C-F}=242.4 \mathrm{~Hz}\right.$, $\left.\mathrm{C}(2) \mathrm{CHF}_{2}\right), 112.87\left(\mathrm{t},{ }^{1} J_{\mathrm{C}-\mathrm{F}}=241.4 \mathrm{~Hz}, \mathrm{C}(4) C H F_{2}\right) . \mathrm{C}_{17} \mathrm{H}_{11} \mathrm{~F}_{4} \mathrm{NS}$ (337): calcd (\%) N 4.15, C 60.53, H 3.29, found N 4.22, C 60.60, H 3.36. MP: $109.9-110.7^{\circ} \mathrm{C}$.

2,4-Bis(difluoromethyl)benzo[h]quinolin-7-ol 3u. The product was prepared according to the general procedure and starting from an activated solution of TFEDMA 4a (1.2 equiv., $0.298 \mathrm{~mL}$, $2.55 \mathrm{mmol}$ ) by $\left(\mathrm{BF}_{3} \bullet \mathrm{Et}_{2} \mathrm{O}\right)$ (1.2 equiv., $0.323 \mathrm{~mL}, 2.55 \mathrm{mmol}$ ) and 5-[(1,1-difluoropropan-2-ylidene)amino]-1-naphthol 1uu (1 equiv., $500 \mathrm{mg}, 2.13 \mathrm{mmol}$ ) in presence of anhydrous acetonitrile. 2,4-Bis(difluoromethyl)benzo[h]quinolin-7-ol $\mathbf{3 u}$ was provided after purification using a gradient of ethyl acetate in pentane (0-5\%) as a brown solid (321.7 mg, 51\%). ${ }^{1 \mathrm{H}}$ NMR $\delta H=8.90\left(1 \mathrm{H}, \mathrm{d},{ }^{3} J_{H-H}=8.3 \mathrm{~Hz}, \mathrm{C}(10) \mathrm{H}\right), 8.39\left(1 \mathrm{H}, \mathrm{d}^{3}{ }^{3} \mathrm{H}_{\mathrm{H}} \mathrm{H}\right.$ $=9.4 \mathrm{~Hz}, \mathrm{C}(5) \mathrm{H}), 8.04(1 \mathrm{H}, \mathrm{s}, \mathrm{C}(3) \mathrm{H}), 7.93\left(1 \mathrm{H}, \mathrm{d},{ }^{3} J_{\mathrm{H}-\mathrm{H}}=9.4 \mathrm{~Hz}\right.$, $\mathrm{C}(6) \mathrm{H}), 7.61\left(1 \mathrm{H}, \mathrm{t}, 3^{3} \mathrm{H}_{-H}=8.0 \mathrm{~Hz}, \mathrm{C}(9) \mathrm{H}\right), 7.25\left(1 \mathrm{H}, \mathrm{t},{ }^{2} J_{H-F}=\right.$ $\left.54.4 \mathrm{~Hz}, \mathrm{C}\left(4-\mathrm{CHF}_{2}\right) \mathrm{H}\right), 7.11\left(1 \mathrm{H}, \mathrm{d},{ }^{3} J_{\mathrm{H}-\mathrm{H}}=7.6 \mathrm{~Hz}, \mathrm{C}(8) \mathrm{H}\right), 6.92(1$ $\left.\mathrm{H}, \mathrm{t},{ }^{2} \mathrm{~J}_{\mathrm{H}-\mathrm{F}}=55.3 \mathrm{~Hz}, \mathrm{C}\left(2-\mathrm{CHF}_{2}\right) \mathrm{H}\right), 5.43(1 \mathrm{H}, \mathrm{br} \mathrm{s}, \mathrm{OH}) \mathrm{ppm} .{ }^{19} \mathrm{~F}$ $\mathrm{NMR} \delta \mathrm{F}=-113.93\left(2 \mathrm{~F}, \mathrm{~d},{ }^{2} \mathrm{~J}_{\mathrm{F}-\mathrm{H}}=55.3 \mathrm{~Hz}, \mathrm{C} 2-\mathrm{CHF}_{2}\right),-114.96(2 \mathrm{~F}$, d, $\left.{ }^{2} J_{F-H}=54.4 \mathrm{~Hz}, \mathrm{C} 4-\mathrm{CHF}_{2}\right) \mathrm{ppm} .{ }^{13} \mathrm{C} \mathrm{NMR} \delta \mathrm{C}=151.71(\mathrm{~s}, \mathrm{C}-7)$, $151.27\left(\mathrm{t},{ }^{2} J_{\mathrm{C}-F}=27.2 \mathrm{~Hz}, \mathrm{C}-2\right), 146.29(\mathrm{~s}, \mathrm{C}-10-\mathrm{C}-\mathrm{C}-\mathrm{N}), 139.47(\mathrm{t}$, $\left.2 J_{C-F}=22.2 \mathrm{~Hz}, \mathrm{C}-4\right), 132.81$ (2 C, s, C-5-C-C-4/C-10-C-CN), 128.23 (s, C-9), 124.51 (s, C-5), 123.57 (s, C-7-C-C-6), 118.94 (s, $\mathrm{C}-6), 117.77(\mathrm{~s}, \mathrm{C}-10), 114.73\left(\mathrm{tt},{ }^{3} \mathrm{~J}_{C-F}=7.6,{ }^{3} \mathrm{~J}_{\mathrm{C}-F}=2.2 \mathrm{~Hz}, \mathrm{C}-3\right)$, $114.60\left(\mathrm{t},{ }^{1} \mathrm{~J}_{\mathrm{C}-\mathrm{F}}=241.0 \mathrm{~Hz}, \mathrm{C}(2) \mathrm{CHF}_{2}\right), 113.24(\mathrm{~s}, \mathrm{C}-5), 113.06(\mathrm{t}$, $\left.{ }^{1} J_{C-F}=242.4 \mathrm{~Hz}, \mathrm{C}(4) C H F_{2}\right)$ ppm. $\mathrm{C}_{15} \mathrm{H}_{9} \mathrm{~F}_{4} \mathrm{NO}$ (295): calcd (\%) $\mathrm{N}$ 4.74, C 61.02, H 3.07, found N 4.82, C 60.87, H 3.14. MP: 139.5 $-141.2^{\circ} \mathrm{C}$.

Functionnalization in position 8 of quinoline derivatives 8-(Bromomethyl)-2,4-bis(difluoromethyl)-7-fluoroquinoline 7. 
Method A: To a solution of 2,4-bis(difluoromethyl)-7-fluoro-8methylquinoline $3 \mathrm{~m}$ (1 equiv., $300 \mathrm{mg}, 1.15 \mathrm{mmol}$ ) and oxone (2 equiv., $349 \mathrm{mg}, 2.3 \mathrm{mmol}$ ) in a mixture of $\mathrm{DCM}(2.7 \mathrm{~mL})$ and $\mathrm{H}_{2} \mathrm{O}(0.3 \mathrm{~mL}$ ) (9:1) was added $\mathrm{KBr}$ (2 equiv., $273 \mathrm{mg}, 2.3 \mathrm{mmol}$ ) at room temperature, and stirred under visible light irradiation for $14 \mathrm{~h}$. Upon addition of $\mathrm{KBr}$ the reaction mixture became brown, then turned into a colourless suspension after $10 \mathrm{~min}$ under light. Saturated aq. solution $\mathrm{Na}_{2} \mathrm{~S}_{2} \mathrm{O}_{3}$ was added to the reaction mixture, and the product was extracted with ethyl acetate. The combined extracts were washed with brine and dried over $\mathrm{Na}_{2} \mathrm{SO}_{4}$. The organic phase was concentrated under reduced pressure.

Method B: To a mixture of 2,4-bis(difluoromethyl)-7-fluoro-8methylquinoline $3 \mathrm{~m}$ (1 equiv., $300 \mathrm{mg}, 1.15 \mathrm{mmol}$ ), $\mathrm{NaIO}_{4}(1.5$ equiv., $368 \mathrm{mg}, 1.72 \mathrm{mmol}$ ) and $\mathrm{LiBr}$ (1.5 equiv., $149 \mathrm{mg}, 1.72$ mmol) was added aq. $\mathrm{H}_{2} \mathrm{SO}_{4}(2 \%)(6 \mathrm{~mL})$. The reaction mixture was heated at $95^{\circ} \mathrm{C}$ for $6 \mathrm{~h}$. The reaction mixture was then cooled to room temperature and extracted with ethyl acetate. The combined organic layers were washed with saturated aq. solution $\mathrm{Na}_{2} \mathrm{~S}_{2} \mathrm{O}_{3}$ then water, dried over anhydrous $\mathrm{Na}_{2} \mathrm{SO}_{4}$ and concentrated under reduced pressure. In both cases the crude reaction mixture was purified by column chromatography using a gradient of ethyl acetate in cyclohexane (0-15\%).

8-(bromomethyl)-2,4-bis(difluoromethyl)-7-fluoroquinoline 7 was obtained as a colourless solid $194 \mathrm{mg}$ (Method A: 63\% / Method B: $50 \%) .{ }^{1} \mathrm{H}$ NMR $\delta \mathrm{H}=8.17\left(1 \mathrm{H}, \mathrm{dd},{ }^{3} J_{\mathrm{H}-\mathrm{H}}=9.4,{ }^{4} J_{\mathrm{H}-\mathrm{F}}=\right.$ $5.7 \mathrm{~Hz}, \mathrm{C}(5) \mathrm{H}), 7.93(1 \mathrm{H}, \mathrm{s}, \mathrm{C}(3) \mathrm{H}), 7.55\left(1 \mathrm{H}, \mathrm{t},{ }^{3} \mathrm{~J}_{\mathrm{H}-\mathrm{H}}=9.1 \mathrm{~Hz}\right.$, $\mathrm{C}(6) \mathrm{H}), 7.14\left(1 \mathrm{H}, \mathrm{t},{ }^{2} \mathrm{~J}_{\mathrm{H}-\mathrm{F}}=54.1 \mathrm{~Hz}, \mathrm{C}\left(4-\mathrm{CHF}_{2}\right) \mathrm{H}\right), 6.86\left(1 \mathrm{H}, \mathrm{t},{ }^{2} \mathrm{~J}_{\mathrm{H}}\right.$ $\left.F=55.0 \mathrm{~Hz}, \mathrm{C}\left(2-\mathrm{CHF}_{2}\right) \mathrm{H}\right), 5.22\left(2 \mathrm{H}, \mathrm{d},{ }^{4} J_{\mathrm{H}-\mathrm{F}}=1.6 \mathrm{~Hz}, \mathrm{C}(8-\right.$ $\left.\left.\mathrm{CH}_{2} \mathrm{Br}\right) \mathrm{H}\right) .{ }^{19} \mathrm{~F} N M R \delta \mathrm{F}=-106.81--106.85(1 \mathrm{~F}, \mathrm{~m}, \mathrm{~F}),-114.12(2$ $\left.\mathrm{F}, \mathrm{d},{ }^{2} \mathrm{~J}_{\mathrm{H}-\mathrm{F}}=54.1 \mathrm{~Hz}, \mathrm{C} 2-\mathrm{CHF}_{2}\right),-114.52\left(2 \mathrm{~F}, \mathrm{~d},{ }^{2} J_{\mathrm{H}-\mathrm{F}}=55.0 \mathrm{~Hz}\right.$, $\left.\mathrm{C}-\mathrm{CHF}_{2}\right) .{ }^{13} \mathrm{C}$ NMR $\delta \mathrm{C}=161.55\left(\mathrm{~d},{ }^{1} J_{C-F}=257.1 \mathrm{~Hz}, \mathrm{C}-7\right), 153.60$ $\left(\mathrm{t},{ }^{2} J_{C-F}=27.7 \mathrm{~Hz}, \mathrm{C}-2\right), 146.39\left(\mathrm{~d},{ }^{3} J_{C-F}=8.3 \mathrm{~Hz}, \mathrm{C}-8-C-\mathrm{N}\right), 140.59$ $\left(\mathrm{td},{ }^{2} J_{C-F}=22.5,5 J_{C-F}=1.5 \mathrm{~Hz}, \mathrm{C}-4\right), 125.95\left(\mathrm{~d},{ }^{2} J_{C-F}=10.7 \mathrm{~Hz}, \mathrm{C}-\right.$ 8), $122.95\left(\mathrm{~d},{ }^{3} J_{C-F}=13.7 \mathrm{~Hz}, \mathrm{C}-5\right), 122.14(\mathrm{~s}, \mathrm{C}-5-\mathrm{C}-\mathrm{C}-4), 119.74$ $\left(d,{ }^{2} J_{C-F}=25.7 \mathrm{~Hz}, \mathrm{C}-6\right), 114.26\left(\mathrm{t},{ }^{1} J_{C-F}=242.4 \mathrm{~Hz}, \mathrm{C}(2) C H F_{2}\right)$, $114.36-114.18(\mathrm{~m}, \mathrm{C}-3), 113.20\left(\mathrm{t},{ }^{1} J_{C-F}=241.7 \mathrm{~Hz}, \mathrm{C}(4) C H F_{2}\right)$, 20.07 (d, ${ }^{3} J_{C-F}=4.6 \mathrm{~Hz}, \mathrm{C}(8) \mathrm{CH}_{2} \mathrm{Br}$ ). $\mathrm{C}_{12} \mathrm{H}_{7} \mathrm{~F}_{5} \mathrm{NBr}(340)$ : calcd (\%) N 4.12, C 42.38, H 2.07 found N 4.22, C 42.33, H 2.68.

8-(Bromomethyl)-7-chloro-2,4-bis(difluoromethyl)quinoline 8

Method A : The same previous procedure was used on the 7chloro-2,4-bis(difluoromethyl)-8-methylquinoline $3 n$ (1 equiv., $300 \mathrm{mg}, 1.08 \mathrm{mmol}$ )

Method B: The same previous procedure was used on the 7chloro-2,4-bis(difluoromethyl)-8-methylquinoline 3n (1 equiv., $302 \mathrm{mg}, 1.09 \mathrm{mmol})$. In both cases the crude reaction mixture was purified by column chromatography using a gradient of ethyl acetate in cyclohexane (0-15\%).

8-(Bromomethyl)-7-chloro-2,4-bis(difluoromethyl)quinoline 8 was obtained as a colourless solid $263 \mathrm{mg}$ (Method A: 68\% / Method B: $55 \%) .{ }^{1} \mathrm{H} N M R \delta H=8.08\left(1 \mathrm{H}, \mathrm{d},{ }^{3} \mathrm{~J}_{\mathrm{H}-\mathrm{H}}=9.1 \mathrm{~Hz}\right.$, $\mathrm{C}(5) \mathrm{H}), 7.96(1 \mathrm{H}, \mathrm{s}, \mathrm{C}(3) \mathrm{H}), 7.75\left(1 \mathrm{H}, \mathrm{d}, 3 J_{\mathrm{H}-\mathrm{H}}=9.1 \mathrm{~Hz}, \mathrm{C}(6) \mathrm{H}\right)$, $7.14\left(1 \mathrm{H}, \mathrm{t},{ }^{2} \mathrm{~J}_{\mathrm{H}-\mathrm{F}}=54.1 \mathrm{~Hz}, \mathrm{C}\left(4-\mathrm{CHF}_{2}\right) \mathrm{H}\right), 6.85\left(1 \mathrm{H}, \mathrm{t},{ }^{2} \mathrm{~J}_{\mathrm{H}-\mathrm{F}}=54.9\right.$ $\left.\mathrm{Hz}, \mathrm{C}\left(2-\mathrm{CHF}_{2}\right) \mathrm{H}\right), 5.36\left(1 \mathrm{H}, \mathrm{s}, \mathrm{C}\left(8-\mathrm{CH}_{2} \mathrm{Br}\right) \mathrm{H}\right) .{ }^{19} \mathrm{~F} N M R \delta \mathrm{F}=-$ $114.27--114.56\left(4 \mathrm{~F}, \mathrm{~m}, \mathrm{C} 2-\mathrm{CHF}_{2} / \mathrm{C}_{4}-\mathrm{CHF}_{2}\right) .{ }^{13} \mathrm{C} \mathrm{NMR} \delta \mathrm{C}=$ $153.37\left(\mathrm{t},{ }^{2} J_{C-F}=27.8 \mathrm{~Hz}, \mathrm{C}-2\right), 145.97(\mathrm{~s}, \mathrm{C}-8-C-\mathrm{N}), 140.63\left(\mathrm{t},{ }^{2} J_{C-}\right.$ $F=22.4 \mathrm{~Hz}, \mathrm{C}-4), 137.78(\mathrm{~s}, \mathrm{C}-7), 135.37$ (s, C-8), 131.05 (s, C-6),
124.80 (s, C-5), 123.76 (s, C-5-C-C-4), $115.04-114.85$ (m, C-3), $114.21\left(\mathrm{t},{ }^{1} J_{C-F}=242.4 \mathrm{~Hz}, \mathrm{C}(2) \mathrm{CHF}_{2}\right), 113.05\left(\mathrm{t},{ }^{1} J_{C-F}=241.8 \mathrm{~Hz}\right.$, $\mathrm{C}(4) \mathrm{CHF}_{2}$ ), 25.08 (s, C(8) $\left.\mathrm{CH}_{2} \mathrm{Br}\right) . \mathrm{C}_{12} \mathrm{H}_{7} \mathrm{~F}_{4} \mathrm{NBrCl}$ (356): calcd (\%) $\mathrm{N}$ 3.93, C 40.42, H 1.98 found N 3.78, C 40.78, H 2.18. MP: 113.3$114^{\circ} \mathrm{C}$.

7-Chloro-2,4-bis(difluoromethyl)quinoline-8-carboxylic acid 9.

To a solution of 8-(bromomethyl)-7-chloro-2,4bis(difluoromethyl)quinoline 8 (1 equiv., $370 \mathrm{mg}, 1.04 \mathrm{mmol}$ ) in aq. $\mathrm{H}_{2} \mathrm{SO}_{4}(70 \%)(3962 \mathrm{mg})$ at $110^{\circ} \mathrm{C}$ was added aq. $\mathrm{HNO}_{3}$ (65\%) (321 mg) dropwise. CAUTION: a release of Brown toxic gaz was observed. The reaction mixture was stirred for $17 \mathrm{~h}$, then the reaction mixture was cooled down and poured into ice. The precipitate was filtered off, washed with water and dried under vacuum. The crude was triturated into pentane to remove all impurities. 7-Chloro-2,4bis(difluoromethyl)quinoline-8-carboxylic acid 9 was obtained as a beige solid $(77.7 \mathrm{mg}, 64 \%) .{ }^{1} \mathrm{H} N M R ~ \delta H=9.55(1 \mathrm{H}, \mathrm{br} \mathrm{s}$, $\mathrm{OH}), 8.22\left(1 \mathrm{H}, \mathrm{d},{ }^{3} \mathrm{~J}_{\mathrm{H}-\mathrm{H}}=9.0 \mathrm{~Hz}, \mathrm{C}(5) \mathrm{H}\right), 8.00(1 \mathrm{H}, \mathrm{s}, \mathrm{C}(3) \mathrm{H})$, $7.84\left(1 \mathrm{H}, \mathrm{d},{ }^{3} J_{H-H}=9.1 \mathrm{~Hz}, \mathrm{C}(6) \mathrm{H}\right), 7.18\left(1 \mathrm{H}, \mathrm{t}, 2 J_{H-F}=53.9 \mathrm{~Hz}\right.$, $\left.\mathrm{C}\left(4-\mathrm{CHF}_{2}\right) \mathrm{H}\right), 6.84\left(1 \mathrm{H}, \mathrm{t},{ }^{2} J_{\mathrm{H}-\mathrm{F}}=54.7 \mathrm{~Hz}, \mathrm{C}\left(2-\mathrm{CHF}_{2}\right) \mathrm{H}\right) .{ }^{19} \mathrm{~F} \mathrm{NMR}$ $\delta \mathrm{F}=-114.47\left(2 \mathrm{~F}, \mathrm{~d},{ }^{2} J_{H-F}=53.9 \mathrm{~Hz}, \mathrm{C} 2-C H F_{2}\right),-115.22(2 \mathrm{~F}, \mathrm{~d}$, $\left.{ }^{2} J_{H-F}=54.7 \mathrm{~Hz}, \mathrm{C} 4-\mathrm{CHF}_{2}\right) .{ }^{13} \mathrm{C} \mathrm{NMR} \delta \mathrm{C}=167.41\left(\mathrm{~s}, \mathrm{C}(8) \mathrm{CO}_{2} \mathrm{H}\right)$, $153.70\left(\mathrm{t},{ }^{2} J_{C-F}=27.8 \mathrm{~Hz}, \mathrm{C}-2\right), 145.41(\mathrm{~s}, \mathrm{C}-8-C-\mathrm{N}), 141.30\left(\mathrm{t},{ }^{2} J_{C-}\right.$ $F=22.7 \mathrm{~Hz}, \mathrm{C}-4), 137.17(\mathrm{~s}, \mathrm{C}-7), 131.93(\mathrm{~s}, \mathrm{C}-6), 130.18$ (s, C-8), $126.57(\mathrm{~s}, \mathrm{C}-5), 123.41(\mathrm{~s}, \mathrm{C}-5-\mathrm{C}-\mathrm{C}-4), 115.48\left(\mathrm{t},{ }^{3} J_{\mathrm{C}-F}=7.9 \mathrm{~Hz}, \mathrm{C}-\right.$ 3), $113.30\left(\mathrm{t},{ }^{1} J_{C-F}=243.4 \mathrm{~Hz}, \mathrm{C}(2) C H F_{2}\right), 112.74\left(\mathrm{t},{ }^{1} J_{C-F}=242.3\right.$ $\mathrm{Hz}, \mathrm{C}(4) \mathrm{CHF}_{2}$ ). $\mathrm{C}_{12} \mathrm{H}_{7} \mathrm{~F}_{4} \mathrm{NBrCl}$ (356): calcd (\%) N 4.55, C 46.80, H 1.95 found N 4.56, C 46.41, H 2.06. MP: $158-159^{\circ} \mathrm{C}$.

7-Chloro-2,4-bis(difluoromethyl)quinoline-8-carbonitrile 10 and Tris(\{[7-chloro-2,4-bis(difluoromethyl)quinolin-8yl]methyl\})amine 11 . To a solution of 7-chloro-2,4bis(difluoromethyl)-8-methylquinoline $3 \mathrm{n}$ (1 equiv., $300 \mathrm{mg}$, $1.08 \mathrm{mmol}$ ) and aq. $\mathrm{HBr}$ (48\%) (3 equiv., $0.368 \mathrm{~mL}, 3.24 \mathrm{mmol}$ ) in $\mathrm{CCl}_{4}\left(4.5 \mathrm{~mL}\right.$ ) was added dropwise aq. $\mathrm{H}_{2} \mathrm{O}_{2}$ (30\%) (2 equiv., $0.17 \mathrm{~mL}, 2.16 \mathrm{mmol}$ ) at room temperature. The suspension became red and the reaction mixture was stirred under sunlamp for $1 \mathrm{~h}$. Then, acetonitrile $(2 \mathrm{~mL})$, aq. $\mathrm{NH}_{3}(35 \%)(50.2$ equiv., $3 \mathrm{~mL}, 54.3 \mathrm{mmol}$ ), and $\mathrm{I}_{2}$ ( 2.5 equiv., $685 \mathrm{mg}, 2.7 \mathrm{mmol}$ ) were added to the mixture at room temperature, and stirred overnight at $60{ }^{\circ} \mathrm{C}$. The reaction mixture was cooled down to room temperature and quenched by saturated aq. Solution $\mathrm{Na}_{2} \mathrm{~S}_{2} \mathrm{O}_{3}$. The mixture was extracted with DCM and the combined organic layers were washed with water and brine, then dried over $\mathrm{Na}_{2} \mathrm{SO}_{4}$ and concentrated under vacuum. 7Chloro-2,4-bis(difluoromethyl)quinoline-8-carbonitrile 10 was isolated after purification using ethyl acetate in cyclohexane (0-15\%) as a yellow solid (91 mg, 29\%). ${ }^{1} \mathrm{H} \mathrm{NMR} \delta \mathrm{H}=8.36(1 \mathrm{H}$, $\left.\mathrm{d},{ }^{3} \mathrm{~J}_{\mathrm{H}-\mathrm{H}}=9.1 \mathrm{~Hz}, \mathrm{C}(5) \mathrm{H}\right), 8.03(1 \mathrm{H}, \mathrm{s}, \mathrm{C}(3) \mathrm{H}), 7.85\left(1 \mathrm{H}, \mathrm{d},{ }^{3} \mathrm{~J}_{\mathrm{H}-\mathrm{H}}=\right.$ $9.1 \mathrm{~Hz}, \mathrm{C}(6) \mathrm{H}), 7.14\left(1 \mathrm{H}, \mathrm{t},{ }^{2} \mathrm{~J}_{\mathrm{H}-\mathrm{F}}=51.0 \mathrm{~Hz}, \mathrm{C}\left(4-\mathrm{CHF}_{2}\right) \mathrm{H}\right), 6.88(1$ $\left.\mathrm{H}, \mathrm{t},{ }^{2} J_{H-F}=51.7 \mathrm{~Hz}, \mathrm{C}\left(2-\mathrm{CHF}_{2}\right) \mathrm{H}\right) .{ }^{19} \mathrm{~F} \mathrm{NMR} \delta \mathrm{F}=-113.56(2 \mathrm{~F}, \mathrm{~d}$, $\left.{ }^{2} J_{H-F}=53.8 \mathrm{~Hz}, \mathrm{C} 2-\mathrm{CHF}_{2}\right),-114.48\left(2 \mathrm{~F}, \mathrm{~d},{ }^{2} J_{H-F}=54.6 \mathrm{~Hz}, \mathrm{C} 4-\right.$ $\left.\mathrm{CHF}_{2}\right) \cdot{ }^{13} \mathrm{C} N M R \delta \mathrm{C}=155.77\left(\mathrm{t},{ }^{2} \mathrm{C}_{\mathrm{C}-\mathrm{F}}=28.3 \mathrm{~Hz}, \mathrm{C}-2\right), 147.88(\mathrm{~s}, \mathrm{C}-$ 8-C-N), $142.63(\mathrm{~s}, \mathrm{C}-7), 141.07\left(\mathrm{t},{ }^{2} J_{C-F}=22.9 \mathrm{~Hz}, \mathrm{C}-4\right), 130.33(\mathrm{~s}$, C-6), 129.10 (s, C-5), 123.23 (s, C-5-C-C-4), 116.48 (t, ${ }^{3} \mathrm{~J}_{\mathrm{C}-F}=7.9$ $\mathrm{Hz}, \mathrm{C}-3), 114.62(\mathrm{~s}, \mathrm{C}(8) C N), 113.66\left(\mathrm{t},{ }^{1} J_{C-F}=242.9 \mathrm{~Hz}\right.$, $\mathrm{C}(2) \mathrm{CHF}_{2}$ ), 113.55 (s C-8), $112.89\left(\mathrm{t},{ }^{1} \mathrm{~J}_{\mathrm{C}-\mathrm{F}}=242.3 \mathrm{~Hz}, \mathrm{C}(4) \mathrm{CHF}_{2}\right.$ ). 
HRMS $\left(E S I+\right.$ ) for $\mathrm{C}_{12} \mathrm{H}_{6} \mathrm{~F}_{4} \mathrm{~N}_{2} \mathrm{Cl}[\mathrm{M}+\mathrm{H}]$ : calcd 289.0150 found 289.0164. MP: $116-116.5^{\circ} \mathrm{C}$.

Tris(\{[7-chloro-2,4-bis(difluoromethyl)quinolin-8-

yl]methyl\})amine $\mathbf{1 1}$ was isolated during the purification of $\mathbf{1 0}$ as a yellow solid $(94.5 \mathrm{mg}, 10 \%) .{ }^{1} \mathrm{H} N M R \quad \delta \mathrm{H}=7.95\left(3 \mathrm{H}, \mathrm{d},{ }^{3} \mathrm{~J}_{\mathrm{H}-}\right.$ $\left.{ }_{H}=9.1 \mathrm{~Hz}, \mathrm{C}(5) \mathrm{H}\right), 7.82(3 \mathrm{H}, \mathrm{s}, \mathrm{C}(3) \mathrm{H}), 7.57\left(3 \mathrm{H}, \mathrm{d},{ }^{3} J_{H-H}=9.1\right.$ $\mathrm{Hz}, \mathrm{C}(6) \mathrm{H}), 7.11\left(3 \mathrm{H}, \mathrm{t}^{2} \mathrm{~J}_{\mathrm{H}-\mathrm{F}}=54.2 \mathrm{~Hz}, \mathrm{C}\left(4-\mathrm{CHF}_{2}\right) \mathrm{H}\right), 6.39(3 \mathrm{H}, \mathrm{t}$, $\left.{ }^{2} J_{H-F}=55.1 \mathrm{~Hz}, \mathrm{C}\left(2-\mathrm{CHF}_{2}\right) \mathrm{H}\right), 4.49\left(6 \mathrm{H}, \mathrm{s}, \mathrm{C}\left(8-\mathrm{CH}_{2}\right) \mathrm{H}\right) .{ }^{19} \mathrm{~F} \mathrm{NMR}$ $\delta \mathrm{F}=-114.15\left(6 \mathrm{~F}, \mathrm{~d},{ }^{2} J_{\mathrm{H}-\mathrm{F}}=55.1 \mathrm{~Hz}, \mathrm{C} 2-\mathrm{CHF}_{2}\right),-114.67(6 \mathrm{~F}, \mathrm{~d}$, $\left.{ }^{2} J_{H-F}=54.2 \mathrm{~Hz}, \mathrm{C} 4-C H F_{2}\right) \cdot{ }^{13} \mathrm{C} \mathrm{NMR} \delta \mathrm{C}=152.45\left(3 \mathrm{C}, \mathrm{t},{ }^{2} J_{C-F}=\right.$ $27.7 \mathrm{~Hz}, \mathrm{C}-2$ ), 147.95 (3 C, s, C-8-C-N), 140.13 (3 C, t, ${ }^{2} \mathrm{~J}_{\mathrm{C}-\mathrm{F}}=22.3$ Hz, C-4), 139.25 (3 C, s, C-7), 136.72 (3 C, s, C-8), 130.92 (3C, s, C-6), 123.33 (3 C, s, C-5), 123.01 (3 C, s, C-5-C-C-4), 114.28 (3 C, $\left.\mathrm{t},{ }^{1} J_{C-F}=242.4 \mathrm{~Hz}, \mathrm{C}(2) C H F_{2}\right), 113.96\left(3 \mathrm{C}, \mathrm{t},{ }^{3} J_{C-F}=7.9 \mathrm{~Hz}, \mathrm{C}-3\right)$, $113.08\left(3 \mathrm{C}, \mathrm{t},{ }^{1} \mathrm{~J}_{\mathrm{C}-F}=241.4 \mathrm{~Hz}, \mathrm{C}(4) \mathrm{CHF}_{2}\right), 50.30(3 \mathrm{C}, \mathrm{s}$, $\mathrm{C}(8) \mathrm{CH}_{2}$ ). HRMS $\left(\mathrm{ESI}+\right.$ ) for $\mathrm{C}_{36} \mathrm{H}_{22} \mathrm{~F}_{12} \mathrm{~N}_{4} \mathrm{Cl}_{3}[\mathrm{M}+\mathrm{H}]$ : calcd 843.0713 found 843.0716 . MP: $120-121^{\circ} \mathrm{C}$.

8-(Benzenesulfinyl)-2,4-bis(difluoromethyl)quinoline 13. $\mathrm{FeCl}_{3}$ (0.03 equiv., $1.59 \mathrm{mg}, 0.00979 \mathrm{mmol}$ ) and 2,4bis(difluoromethyl)-8-(phenylsulfanyl)quinoline 3t (1 equiv., $110 \mathrm{mg}, 0.326 \mathrm{mmol}$ ) were dissolved in acetonitrile $(1 \mathrm{~mL})$ and stirred for $5 \mathrm{~min}$. To this solution was added $\mathrm{H}_{5} \mathrm{IO}_{6}$ (1.1 equiv., $81.8 \mathrm{mg}, 0.359 \mathrm{mmol}$ ) at once. The yellow suspension, was stirred at room temperature for $3 \mathrm{~h}$. The reaction was quenched by the addition of a saturated aq. solution of $\mathrm{Na}_{2} \mathrm{~S}_{2} \mathrm{O}_{3}$ and extracted with DCM. Organic layers were dried over $\mathrm{Na}_{2} \mathrm{SO}_{4}$ and concentrated under vacuum. 8(Benzenesulfinyl)-2,4-bis(difluoromethyl)quinoline 13 was obtained as a sticky brown solid $(91.2 \mathrm{mg}, 79 \%) .{ }^{1} \mathrm{H} \mathrm{NMR} \delta \mathrm{H}=$ $8.61\left(1 \mathrm{H}, \mathrm{dd},{ }^{3} J_{\mathrm{H}-\mathrm{H}}=7.3,{ }^{4} J_{\mathrm{H}-\mathrm{H}}=1.2 \mathrm{~Hz}, \mathrm{C}(7) \mathrm{H}\right), 8.22\left(1 \mathrm{H}, \mathrm{dd},{ }^{3} J_{\mathrm{H}-}\right.$ $\left.H=8.5,{ }^{4} J_{H-H}=1.3 \mathrm{~Hz}, \mathrm{C}(6) \mathrm{H}\right), 7.96\left(1 \mathrm{H}, \mathrm{d},{ }^{3} J_{H-H}=7.5 \mathrm{~Hz}, \mathrm{C}(5) \mathrm{H}\right)$, $7.93(1 \mathrm{H}, \mathrm{s}, \mathrm{C}(3) \mathrm{H}), 7.91-7.87\left(2 \mathrm{H}, \mathrm{m}, \mathrm{C}\left(2^{\prime}, 6^{\prime}\right) \mathrm{H}\right), 7.41-7.31$ $\left(3 \mathrm{H}, \mathrm{m}, \mathrm{C}\left(3^{\prime}, 4^{\prime}, 5^{\prime}\right) \mathrm{H}\right), 7.14\left(1 \mathrm{H}, \mathrm{t},{ }^{2} J_{\mathrm{H}-\mathrm{F}}=54.1 \mathrm{~Hz}, \mathrm{C}\left(4-\mathrm{CHF}_{2}\right) \mathrm{H}\right)$, $6.81\left(1 \mathrm{H}, \mathrm{t},{ }^{2} J_{H-F}=55.0 \mathrm{~Hz}, \mathrm{C}\left(2-\mathrm{CHF}_{2}\right) \mathrm{H}\right) .{ }^{19} \mathrm{~F} \mathrm{NMR} \delta \mathrm{F}=-113.77-$ $-115.99\left(2 \mathrm{~F}, \mathrm{~m}, \mathrm{C} 4-\mathrm{CHF}_{2}\right),-113.36--117.48\left(2 \mathrm{~F}, \mathrm{~m}, \mathrm{C} 4-\mathrm{CHF}_{2}\right.$ ). ${ }^{13} \mathrm{C} N M R \delta C=152.39\left(\mathrm{dd},{ }^{2} J_{C-F}=28.2,26.2 \mathrm{~Hz}, \mathrm{C}-2\right), 145.60(\mathrm{~s}$, C-8-C-N), 145.42 (s, C-1'), $143.42(\mathrm{~s}, \mathrm{C}-8), 140.52\left(\mathrm{t},{ }^{2} \mathrm{~J}_{\mathrm{C}-\mathrm{F}}=22.5\right.$ $\mathrm{Hz}, \mathrm{C}-4$ ), 131.28 (s, C-4'), 129.60 (s, C-5), 129.09 (2 C, s, C-3', 5'), 126.66 (s, C-7), 125.93 (2 C, s, C-2',6'), 124.93 (s, C-5-C-C-4), $115.39-115.08(\mathrm{~m}, \mathrm{C}-3), 113.45\left(\mathrm{t},{ }^{1} J_{\mathrm{C}-F}=243.4 \mathrm{~Hz}, \mathrm{C}(4) C H F_{2}\right)$, $112.79\left(\mathrm{t},{ }^{1} J_{C-F}=241.9 \mathrm{~Hz}, \mathrm{C}(2) \mathrm{CHF}_{2}\right) . \mathrm{C}_{17} \mathrm{H}_{11} \mathrm{~F}_{4} \mathrm{NOS}(353)$ : calcd (\%) N 3.96, C 57.79, H 3.14, found N 3.94, C 57.64, H 3.32.

\section{Acknowledgements}

The CNRS (Centre National de la Recherche Scientifique), Bayer Cropscience AG (Monheim) and Bayer S.A.S (Lyon) are deeply acknowledged for their financial support. The analytical platform of the University of Strasbourg is warmly thanked for help with mass spectroscopy, elemental analysis and crystallography.

\section{Notes and references}

1 J. P. Bégué and D. Bonnet-Delpon, Bioorganic and Medicinal Chemistry of Fluorine, John Wiley \& Sons, Hoboken, New Jersey, 2008.
2 I. Ojima, Fluorine in Medicinal Chemistry and Chemical Biology, Wiley-Blackwell 2009.

3 P. Jeschke, in Modern Methods in Crop Protection Research, eds. P. Jeschke, W. Krämer, U. Schirmer and M. Witschel, Wiley-VCH, Weinheim, Germany, 2012, ch. 4, pp. 73-128.

4 P. Jeschke, ChemBioChem, 2004, 5, 571-589.

5 P. Jeschke, E. Baston and F. R. Leroux, Mini-Rev. Med. Chem., 2007, 7, 1027-1034.

6 K. L. Kirk, Org. Proc. Res. Dev., 2008, 12, 305-321.

7 K. Müller, C. Faeh and F. Diederich, Science, 2007, 317, 18811886.

8 J. Wang, M. Sánchez-Roselló, J. L. Aceña, C. del Pozo, A. E. Sorochinsky, S. Fustero, V. A. Soloshonok and H. Liu, Chem. Rev., 2014, 114, 2432-2506.

9 D. O'Hagan and D. B. Harper, J. Fluorine Chem., 1999, 100 127-133.

$10 \mathrm{~K}$. Uneyama, Fundamentals in Organic Fluorine Chemistry, Blackwell, Oxford, 2006.

11 W. K. Hagmann, J. Med. Chem., 2008, 51, 4359-4369.

12 P. Jeschke, Pest. Manag. Sci., 2010, 66, 10-27.

$13 \mathrm{G}$. Theodoridis, in Advances in Fluorine Science, ed. A. Tressaud, Elsevier B.V, 2006, vol. 2, ch. 4, pp. 120-175.

14 J.-P. Bégué, in Chimie bioorganique et médicinale du fluor, ed. E. C. ÉDITIONS, 2005, p. 384.

15 M. M. Ghorab, F. A. Ragab, H. I. Heiba, R. K. Arafa and E. M. El-Hossary, Med. Chem. Res., 2010, 20, 388-400.

16 F. Leroux, P. Jeschke and M. Schlosser, Chem. Rev., 2005, 105, 827-856.

17 P. V. Reddy, in Organofluorine Compounds in Biology and Medicine, Elsevier, 2015, pp. 1-27.

18 S. Purser, P. R. Moore, S. Swallow and V. Gouverneur, Chem. Soc. Rev., 2008, 37, 320-330.

19 A. M. Thayer, Chem. Eng. News, 2006, 84, 15-24.

20 The Pesticide Manual: A World Compendium, British Crop Protection Council., Alton edn., 2012.

21 S. M. Prajapati, K. D. Patel, R. H. Vekariya, S. N. Panchal and H. D. Patel, RSC Adv., 2014, 4, 24463-24476.

22 Y. Li and W. Gao, Beilstein J. Org. Chem., 2010, 6, 966-972.

23 H. Kumar, A. Chattopadhyay, R. Prasath, V. Devaraji, R. Joshi, P. Bhavana, P. Saini and S. K. Ghosh, J. Phys. Chem. B, 2014, 118, 7257-7266.

24 G. Shan, X. Sun, Q. Xia and Y. Rao, Org. Lett., 2011, 13, 57705773.

25 R. E. Lutz, C. J. Ohnmacht and A. R. Patel, J. Med. Chem., 1971, 14, 926-928.

26 J. Mulero, G. Martinez, J. Oliva, S. Cermeno, J. M. Cayuela, P. Zafrilla, A. Martinez-Cacha and A. Barba, Food Chem., 2015, 180, 25-31.

27 M. Kidwai, K. R. Bhushan, P. Sapra, R. K. Saxena and R. Gupta, Bioorg. Med. Chem., 2000, 8, 69-72.

28 Y.-M. Wu, Y. Li and J. Deng, J. Fluorine Chem., 2006, 127, 223-228.

29 A. R. Surrey and H. F. Hammer, J. Am. Chem. Soc., 1946, 68, 113-116.

30 W. S. Johnson and B. G. Buell, J. Am. Chem. Soc., 1952, 74, 4513-4516.

31 V. Nenajdenko, in Fluorine in Heterocyclic Chemistry Volume 2, ed. V. Nenajdenko, Springer International Publishing, 2014.

32 V. A. Petrov, S. Swearingen, W. Hong and W. Chris Petersen, J. Fluorine Chem., 2001, 109, 25-31.

33 N. N. Yarovenko and M. A. Raksha, Zh. Obshch. Khim., 1959, 29, 2159-2163.

34 N. N. Yarovenko and M. A. Raksha, J. Gen. Chem. USSR (Engl. Transl.), 1959, 29, 2125-2128.

35 A. Takaoka, H. Iwakiri and N. Ishikawa, Bull. Chem. Soc. Jpn., 1979, 52, 3377-3380.

36 V. A. Petrov, Adv. Org. Synth., 2006, 2, 269-290. 
37 A. Vilsmeier and A. Haack, Ber. Dtsch. Chem. Ges., 1927, 60, 119-122.

38 C. Wakselman and M. Tordeux, J.C.S. Chem. Comm, 1975, 956-956.

39 WO Pat., 2008022777, 2008.

40 A. Takaoka, K. Iwamoto, T. Kitazume and N. Ishikawa, J. Fluorine Chem., 1979, 14, 421-428.

41 E. Schmitt, B. Rugeri, A. Panossian, J.-P. Vors, S. Pazenok and F. R. Leroux, Org. Lett., 2015, 17, 4510-4513.

42 F. Giornal, G. Landelle, N. Lui, J.-P. Vors, S. Pazenok and F. R. Leroux, Org. Proc. Res. Dev., 2014, 18, 1002-1009.

43 F. Giornal, S. Pazenok, L. Rodefeld, N. Lui, J.-P. Vors and F. R. Leroux, J. Fluorine Chem., 2013, 152, 2-11.

44 WO Pat., 20141877732014.

45 E. Schmitt, G. Landelle, J.-P. Vors, N. Lui, S. Pazenok and F. R. Leroux, Eur. J. Org. Chem., 2015, 6052-6060.

46 WO Pat., 20151445782015.

47 E. Schmitt, A. Panossian, J.-P. Vors, C. Funke, N. Lui, S. Pazenok and F. R. Leroux, Chem. Eur. J., 2016, in press.

48 K. T. Finley, in Kirk-Othmer Encyclopedia of Chemical Technology, John Wiley \& Sons, Inc., 2000, p. 32.

49 T. L. Gilchrist, J. Chem. Soc., Perkin Trans. 1, 2001, 24912515.

50 A. Roe and G. F. Hawkins, J. Am. Chem. Soc., 1949, 71, 17851786.

51 J. Hamer, W. J. Link, A. Jurjevich and T. L. Vigo, Recl. Trav. Chim. Pays-Bas, 1962, 81, 1058-1060.

52 R. D. Chambers, D. Holling, G. Sandford, H. Puschmann and J. A. K. Howard, J. Fluorine Chem., 2002, 117, 99-101.

53 R. D. Chambers, D. Holling, G. Sandford, A. S. Batsanov and J. A. K. Howard, J. Fluorine Chem., 2004, 125, 661-671.

54 M. Oishi, H. Kondo and H. Amii, Chem. Commun., 2009, 1909-1911.

55 H. Yanai, H. Mimura, K. Kawada and T. Taguchi, Tetrahedron, 2007, 63, 2153-2160.

56 H. Keller and M. Schlosser, Tetrahedron, 1996, 52, 46374644.

57 D. M. Volochnyuk, A. O. Pushechnikov, D. G. Krotko, D. A. Sibgatulin, S. A. Kovalyova and A. A. Tolmachev, Synthesis, 2003, 1531-1540.

58 N. S. Boltacheva, V. I. Filyakova and V. N. Charushin, Russ. J. Org. Chem., 41, 1452-1457.

59 K. Uneyama, J. Fluorine Chem., 1999, 97, 11-25.

60 M. G. Mormino, P. S. Fier and J. F. Hartwig, Org. Lett., 2014, 16, 1744-1747.

61 B. Duda, S. N. Tverdomed, B. S. Bassil and G.-V. Röschenthaler, Tetrahedron, 2014, 70, 8084-8096.

62 J. C. Sloop, C. L. Bumgardner and W. D. Loehle, J. Fluorine Chem., 2002, 118, 135-147.

63 J. C. Sloop, J. Phys. Org. Chem., 2009, 22, 110-117.

64 F. Zhao, X. Yang and J. Liu, Tetrahedron, 2004, 60, 99459951.

65 S. Perrone, F. Rosato, A. Salomone and L. Troisi, Tetrahedron, 2013, 69, 3878-3884.

66 A. Combes, Bull. Soc. Chim. Fr., 1888, 49, 89-92.

67 CCDC 1487974 (3aiii) and 1487975 (9) contain the supplementary crystallographic data for this paper. These data are provided free of charge by The Cambridge Crystallographic Data Centre.

68 B. Wuerzer and R. Berghaus, presented in part at the 10th Asian-Pacific Weed Science Society Conference, Part. 1, Thailand 1985.

69 DE Pat., 3233089, 1984.

70 K. Moriyama, M. Takemura and H. Togo, Org. Lett., 2012, 14, 2414-2417.

71 T. M. A. Shaikh, L. Emmanuvel and A. Sudalai, J. Org. Chem., 2006, 71, 5043-5046.
72 Y. Kawagoe, K. Moriyama and H. Togo, Eur. J. Org. Chem., 2014, 4115-4122.

73 S. Kim, K. Nehru, S. S. Kim, D. W. Kim and H. C. Jung, Synthesis, 2002, 2484-2486. 